[23] M. J. E. Golay, "Monochromaticity and noise in a regenerative electric oscillator," Proc. IRE, vol. 48, pp. 1473-1477, Aug. 1960.

[24] K. H. Sann, "Phase stability of oscillators," Proc. IRE, vol. 49, pp. 527-528, Feb. 1961.

[25] L. P. Malling, "Phase-stable oscillators for space communications, including the relationship between the phase noise, the spectrum, the short-term stability and the $Q$ of oscillators," Proc. IRE, vol. 50, pp. 1656-1664, July 1962 .

[26] L. S. Cutler, "A frequency standard of exceptional spectral purity and long-term stability," Hewlett-Packard Co., July 1961.

[27] W. R. Atkinson, L. Fey, and J. Newman, "Spectrum analysis of extremely low frequency variations of quartz oscillators," Proc. IEEE, vol. 51, p. 379, Feb. 1963.

[28] H. P. Stratemeyr, "The stability of standard-frequency oscillators," The General Radio Experimenter, vol. 38, pp. 1-16, June 1964.

[29] B. B. Mandelbrot, "Sporadic random functions and conditional spectral analysis: self-similar examples and limits," in Proc. 5th Berkeley Symp. on Mathematical Statistics and Probability, Berkeley, and Los Angeles, Univ. California Press, 1967, pp. 155-178.

[30] -, "Some noises with $1 / f$ spectrum, a bridge between direct current and white noise," IEEE Trans. Inform. Theory, vol. IT 13, pp. 289-298, Apr. 1967.

[31] R. Vessot, L. Mueller, and J. Vanier, "The specification of oscillator characteristics from measurements made in the frequency domain," Proc. IEEE, vol. 54, pp. 199-207, Feb. 1966.

[32] D. Halford, "A general mechanical model for $|f|^{\alpha}$ spectral density random noise with spectral reference to tlicker noise $1 /|f|$," Proc. IEEE, vol. 56, pp. 251-258, Mar. 1968.

[33] A. L. McWhorter, " $a / f$ noise and related surface effects in germanium," M.I.T. Research Lab of Electronics, Lexington, MA, Tech. Rep. 295, May 20, 1955.

[34] W. E. Leavitt, "Shipboard satellite communication sets AN/WSC$2(X N-1)(V)$, phase noise specification considerations," 5430 169A, NRL Prob. R01-36, Aug. 2, 1972.

[35] -, "Shipboard satelite communication sets AN/WSC-2(XN-1) (V), phase noise specification considerations," 5430-59A, NRL Prob. R01-36, Mar. 10, 1972.

[36] "Precision frequency measurements," AN-116, Hewlett-Packard Co., July 1969.

[37] "The stability of standard frequency oscillators," General Radio Experimenter, vol. 38, pp. 1-16, June 1964.

[38] M. A. Caloyannides, "A mathematical model and experimental investigation of microcycle spectral estimates of semiconductor flicker noise," Ph.D. dissertation, California Inst. Technol., Pasadena, 1972.

[39] C. B. Searles, R. Ashley, and F. M. Palka, "The measurement of oscillator noise at microwave frequencies," IEEE Trans. Microwave Theory Tech., vol. MTT-16, pp. 753-760, Sept. 1968.

[40] M. Lighthill, Introduction to Fourier Analysts and Generalized Functions. London, England: Cambridge Univ. Press, 1962, pp. 15-57.

\title{
Waves in Active and Passive Periodic Structures:
}

\section{A Review}

\author{
CHARLES ELACHI, MEMBER, IEEE
}

\begin{abstract}
The theory and recent applications of waves in periodic structures are reviewed. Both the Floquet and coupled waves approach are anslyzed in some detail. The theoretical part of the paper includes wave propagntion in unbounded and bounded active or passive periodic media, wave scattering from periodic boundaries, source radintion (dipole, Cerenkov, transition, and Smith-Purcell) in periodic media, and pulse transmission through a periodic slab. The applications part covers the recent development in a veriety of fields: distributed feedback oscillators, filters, mode converters, couplers, second-harmonic generators, deflectors, modulators, and transducers in the fields of integrated optics and integrated aurface acoustics. We also review the work on insect compound eyes, mechanical structures, ocen waves, pulse compressions, temperature waves, and cholesteric liquid crystals. Particles interaction with crystals is briefly reviewed, especially in the case of zeolite crystals and superiattices. Recent advances in fabrication techniques for very fine gratings are also covered. Finnlly, speculations about future problems and development in the field of waves in periodic structures are given.
\end{abstract}

Manuscript received January 13, 1976; revised June 21, 1976. The submission of this paper was encouraged after review of an advanced proposal. This paper presents the results of one phase of research carried out at the Jet Propulsion Laboratory, California Institute of Technology, under Contract NAS7-100, sponsored by the National Aeronautics and Space Administration. This work has also been partly supported by the Office of Naval Research.

The author is with the Jet Propulsion Laboratory, Cahifornia Institute of Technology, Pasadena, CA 91103, and UCLA, Los Angeles, CA.

\section{INTRODUCTION}

7 HE PROPAGATION of waves in periodically stratified media was discussed as early as 1887 by Lord Rayleigh [1], who recognized that this problem was characterized by the Hill and Mathieu differential equations. Even earlier in the 19 th century a number of scientists have investigated wave propagation in lattices. Cauchy, Baden-Powell, and Kelvin [2] discussed lattices that consist of identical particles. Kelvin then proceeded to devise a theory of dispersion for a 2-particle lattice and a mechanical model of it was built by Vincent [3]. At the end of the Nineteenth century and early Twentieth century a number of scientists (Vaschy, Pupin, Campbell) used periodic networks to develop electric filters.

In 1928, Strutt and Van der Pol [4], [5], analyzed the problem of an atomic grating subject to a periodic sinusoidal potential, and the behavior of particles in force fields that are characterized by sinusoidal and rectangular periodic variations. In the same year, Bloch [6] generalized the results of Floquet [7] to the use of partial differential equations with periodic coefficients. The solutions, called the Bloch waves, formed the basis of the theory of electrons in crystals, i.e., of the theory of solids and energy bands [8]-[12]. 


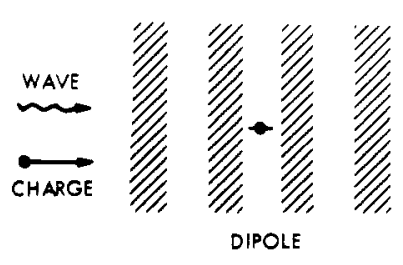

(e)

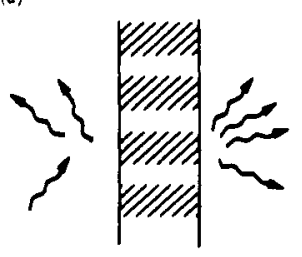

(i)

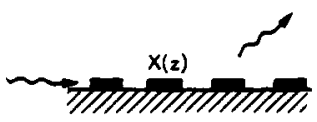

(m)

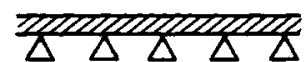

(p)

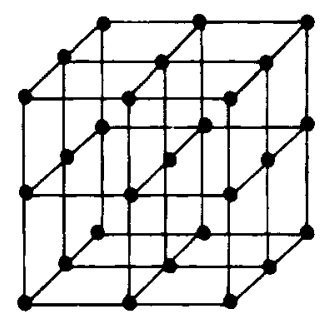

(b)

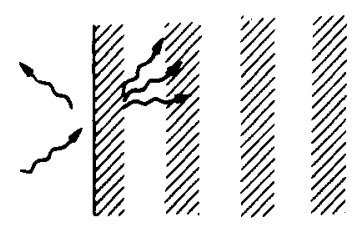

(4)

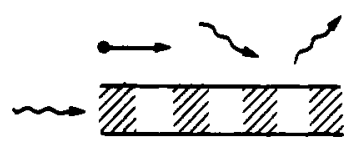

(i)

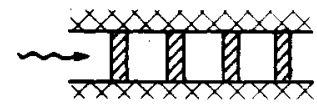

(n)

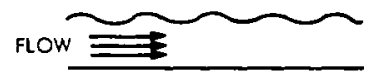

(q)

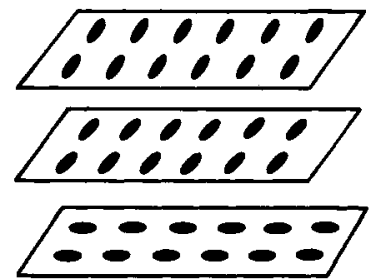

(c)

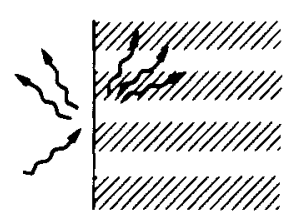

(d)

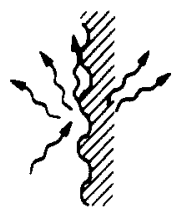

(g)

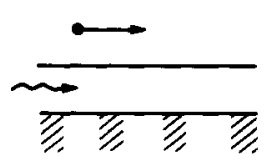

(k)

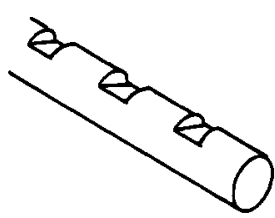

(h)

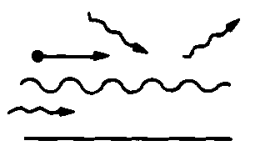

(1)

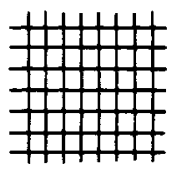

(o)

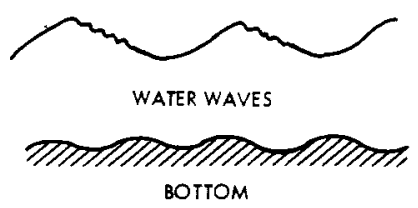

(r)

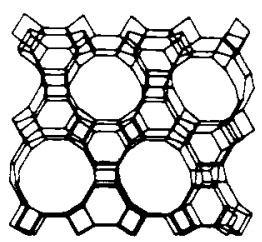

Fis. 1. Periodic structure configurations reviewed in this paper. (a) Waves and particles in an unbounded periodic medium. (b), (c) Wave scattering from a periodic half-space. (d) Wave scattering from a periodic boundary. (e) Wave scattering by a thick grating. (f), (s), (h) Waves in periodic guides and particles moving near a periodic structure. (i) Waveguiding and radiation on a surface with periodic impedance. (j) Guide with periodic loading. (k) Corrugated fiber. (i) Twodimensional periodic mechanical meah. (m) Flexural waves in periodically supported burns. (n) Acoustic waves and flow in a periodic duct. (o) Water waves on a periodic bottom. (p), (q), (r) Waves and particles in simple crystals, cholesteric liquid crystak, and zeolite crystals, respectively.

Interest was also very strong in the field of optical multilayers [13]-[15], which have many applications: filters, antireflection films, beam splitters, and polarizers. The theory of stratified optical thin films was elegantly and considerably investigated by Abeles [16]. A detailed and comprehensive review of the work on waves in periodic structures as of the late 40's can be found in Brillouin's book Wave Propagation in Periodic Structures [17].

In the 1950's the interest in periodic structures came mainly from the fields of slow wave structures and antennas. The study of slow wave structures was mainly stimulated by the development of microwave tubes where a periodic structure is used to slow the wave, which could then couple to the relatively slow electron beam [18]-[22]. The structure most often used was the helix in the different forms: Sheath helix [22], tape helix [23], [24], and multifilar helix [24], [25]. Other structures which were also studied in detail for slow wave guiding and filtering were the tape ladder line [24]-[26] and ridge waveguide [24], [27], [28] .

The investigations of the properties of traveling wave periodically loaded antennas was stimulated by the successful experimental design of the cigar antenna [29], [30] which utilizes a modulated disk on rod structures. The theory of guiding structures with periodic modulation of the surface reactance was then developed [31]-[33]. Periodic loading of a basically slow-wave structure produces a complex wave 
which continuously radiates power, but with the bulk of the energy being bound. This permits the realization of a large effective aperture [32]-[33]. An excellent review, with references, of the work on traveling-wave antenna is given by Hessel [33].

In the 1960's, the main emphasis in the field of periodic structures was toward: 1) exact solution of the electromagnetic wave equation in sinusoidally periodic and laminar media $[34]-[38]$; 2) wave propagation in time and space-time periodic media [39]-[45]; and 3) localized source radiation (dipoles, linear sources and moving charges ) [46]-[52]. Also of importance was the use of interdigital transducers in the field of integrated acoustics [53].

In the early 1970's, new technological advances in the development of passive and active thin-film optical waveguides and the fabrication of solid state, optical, and acoustical gratings had generated a new interest in, and gave a strong impetus to, the field of waves in periodic structures. The interest was mainly stimulated by the fact that new exotic materials, with a wide range of properties (nonlinear, piezoelectric, anisotropic, pyroelectric magnetoelastic, magnetooptic, electrooptic, etc.) can now be used in different forms (bulk, thin films, fibers, etc.) and with very fine periodicities to support electro-magnetic, acoustic or electron waves. Active materials were used to develop distributed feedback lasers [54]-[98]. Periodic nonlinear materials were proposed and used for parametric interaction [99]-[103], and highmobility semiconductors were suggested to develop surface or bulk sources for optical, IR, magnetic, and acoustic waves [104]-[107]. Work on the properties of periodic structures was also active in the fields of structural engineering, classical acoustic, liquid crystals, and insect vision. In Fig. 1, we show a variety of periodic structures that have been studied by numerous authors and that will be reviewed in this paper.

Periodic structures are widely encountered in nature in the form of crystals. They can also be simply generated by a standing wave, i.e., an acoustic wave in a fluid or solid, or an electromagnetic wave in a nonlinear or active medium. Finally, large periodic structures can be developed by just simply repeating a basic unit. These were factors in generating the interest of scientists to study their characteristics. However, two special properties made these structures so unique and important: 1) their eigenmodes consist of an infinite number of space-harmonics with phase velocities varying from zero to infinity; and 2) they can support propagating waves only in well-specified propagation bands.

The uniqueness of the first property is that it allows the periodic structure to support waves that have a very low phase velocity and therefore can be efficiently coupled to relatively slowly moving charges or sources. It also allows the coupling of different types of waves, or similar waves in different modes, without requiring them to have inherently identical wave vectors (in the absence of the periodicity). In other words, the periodic structure has an inherent wave vector ( $K=2 \pi / \Lambda$, where $\Lambda$ is the period of the structure) that is adjustable by the designer and can be used to conserve the momentum (or the wave vector) in the coupling between any two waves.

The second property is commonly known as the distributed feedback (Bragg reflection), which is a result of the cumulative reflection from each unit cell in the structure. As we shall see later, in certain frequency bands the propagation-wave vector can only be complex. This implies that a wave propagating in the structure with a frequency in the stopband will encounter successive reflection, i.e., "distributed feedback," and thus cannot extend far away from its source. This is the reason for the presence of forbidden bands in crystals.

All types of waves exhibit the above properties when they propagate in a periodic structure. The wave could be an acoustic, electromagnetic, magnetoelastic, plasma, electron, flexural, or water wave. The structure could have a periodic boundary, a periodic support, or a periodic bulk parameter (i.e., index of refraction, plasma density, electric potential, nonlinearity constant, gain, density, etc.) The only requirement is that the propagation properties of the wave are somehow related to the perturbed parameter.

In this review paper we will discuss analytically and physically the unique properties, review the theoretical and experimental work in the last 15 years, and speculate on some future developments in the field of waves in periodic media. In Section II, we will use, with no loss of generality, the propagation of an electromagnetic wave in an unbound periodically modulated medium as an example to derive and explain the unique properties of periodic structures.

The exact Floquet approach and also the approximate but simple coupled modes approach are analyzed. In Section III, we include the effect of boundaries. In Section IV, we analyze the case of periodic boundaries. The cases of sources and transients are discussed in Sections V and VI.

Active periodic media and their applications in a wide range of fields are studied in detail in Section VII. Section VIII addresses the recent applications of passive periodic structures. The wide field of electrons in crystals is briefly reviewed in Section IX. The fabrication techniques are presented in Section $X$ and speculations for future development are in Section XI. This paper does not address the work on waves in space-time and temporal periodic structures which require in itself a special review paper.

Throughout this paper, an exp $(-i \omega t)$ time dependence is assumed.

\section{Waves in an Unbound Periodic Medium}

The wave equation in a symmetrically periodic medium can be reduced to a differential equation of the form (Appendix A)

$$
\begin{gathered}
\frac{d^{2} \psi}{d z^{2}}+f(z) \psi=0 \\
f(z)=f\left(z+\frac{2 \pi}{K}\right)=\sum_{n=0}^{\infty} a_{n} \cos (n K z)
\end{gathered}
$$

where $a_{n}$ are related to the Fourier coefficients of the periodicity function and to the wavenumber $k=2 \pi / \lambda, \lambda$ is the wavelength of the propagating wave and $\Lambda=2 \pi / K$ is the period of the medium. $a_{0}$ is also related to the transverse wave vector whenever it exists. The solutions of (1) are the Hill functions of which Mathieu's functions are a special case (when $a_{n}=0$ for $n \neq 0,1)$. The Hill equation also applies for $f(z)$ odd. The general solution can be written in a Floquet form

$$
\psi=\exp (i \kappa z) A(z)
$$

where $A(z)$ is a periodic function, and $k$ is termed the characteristic exponent and is a single valued function of the $a_{n}$ 's. The periodic function $A(z)$ may next be expanded in a Fourier series. The solution can then be written as

$$
\psi=\sum_{n=-\infty}^{n=+\infty} A_{n} \exp [i(K+n K) z]
$$


The different components of $\psi$ are called the space harmonics of the propagating wave, in analogy to the familiar time harmonic expansion for an arbitrary function in time. The values of $\kappa_{n}=\kappa+n K$ represent physically the propagation wave numbers of these space harmonic contributions to the total field. The space harmonics do not exist independently. They are portions of a total solution.

Introducing (4) into the wave equation, one obtains

$$
\begin{aligned}
\sum_{n}\left[-(\kappa+n K)^{2} A_{n}+\frac{1}{2} \sum_{m} a_{m}\left(A_{n-m}+A_{n+m}\right)\right] \\
\cdot \exp [i(\kappa+n K) z]=0 .
\end{aligned}
$$

Since the above relation must hold for any value of $z$, then (5) reduces to an infinite set of homogeneous equations

$$
\begin{aligned}
-2(\kappa+n K)^{2} A_{n}+\sum_{m} a_{m}\left(A_{n-m}+A_{n+m}\right) & \\
& =0, \quad n=0, \pm 1, \ldots
\end{aligned}
$$

which can be written in a matrix form

$$
|| M|| \cdot|A|=0 \text {. }
$$

The solution will be nontrivial if

$$
\operatorname{det}\|M\|=0 \text {. }
$$

This is the dispersion relation which gives the value of $\kappa$ as a function of the $a_{n}$ 's. The solution of (7) would then give the relative values of the space harmonics, i.e., $A_{n} / A_{0}$. The value of $A_{0}$ itself is determined from the boundary or source conditions. An analytical solution for (8) was given by Hill, which relates the free space wave number $k$ to the characteristic exponent $\kappa[35]$.

In the simple case where $a_{n}=0$ for $n \neq 0,1,(6)$ reduces to

$$
D_{n} A_{n}+A_{n+1}+A_{n-1}=0
$$

where

$$
D_{n}=2 \frac{a_{0}-(\kappa+n K)^{2}}{a_{1}} .
$$

Applying an iterative process on (9) one obtains the continued fractions [34]

$$
\begin{aligned}
& \frac{A_{n}}{A_{n-1}}=-\frac{1}{D_{n}}-\frac{1\rfloor}{\mid D_{n+1}}-\frac{1}{\mid D_{n+2}}-\cdots \\
& \frac{A n}{A_{n+1}}=-\frac{1}{\mid D_{n}}-\frac{1}{\mid D_{n-1}}-\frac{1}{\mid D_{n-2}}-\cdots
\end{aligned}
$$

which when combined yield

$D_{n}=\frac{1}{D_{n+1}}-\frac{1}{\mid D_{n+2}}-\frac{1}{\mid D_{n+3}}-\cdots+\frac{1}{D_{n-1}}-\frac{1}{\mid D_{n-2}}-\cdots$

This expression is another form of the dispersion relation. The above continued fractions can be shown [7] to converge if $\left|D_{n}\right| \geqslant 2$ for $n \geqslant N$ where $N$ is a finite integer. An inspection of $D_{n}$ shows that this condition is usually satisfied.

The relation between $k, k$, and the parameters of the perturbation can be illustrated in the form of a stability diagram, which is customary in the study of Mathieu's equation. Fig. 2 shows the stability diagram for the case where $a_{n}=0$ for $n \neq 0,1$. The unshaded areas are the so-called "stable regions"

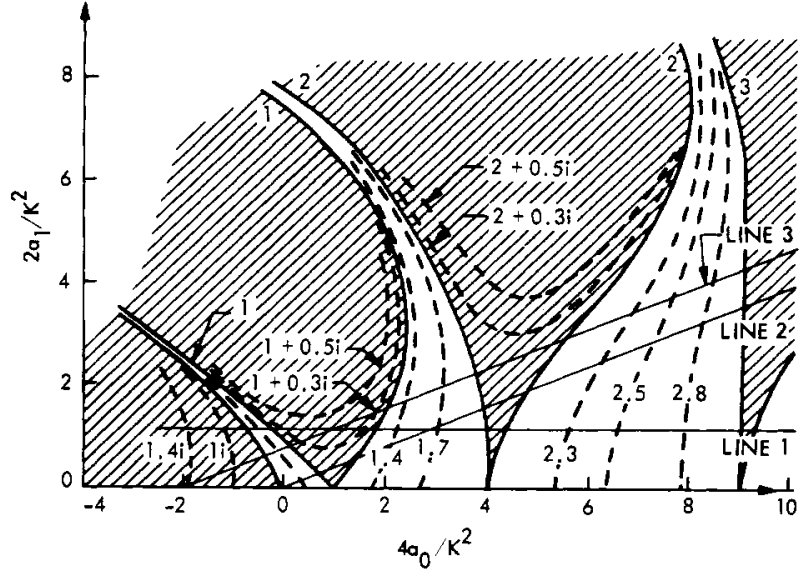

Fig. 2. Stability diagram for the case of Mathieu's equation. The continuous lines correspond to the boundaries separating the regions of complex and real solution. The dashed lines correspond to a fixed solution for $\kappa$. Line 1 corresponds to the case of fixed $K$ and $a$ (i.e., perturbation magnitude and signal frequency) while $a_{0}$ varies; i.e., transverse wave vector. Line 2 corresponds to the case of a wave incident on a periodic half space where the perturbation and the incidence angle are fixed. A change in these two parameters would lead to a change in the slope of the line. Line 3 corresponds to the case of a wave in a modulated guide, where the transverse wave vector is fixed and the perturbation is fixed [ 34 ].

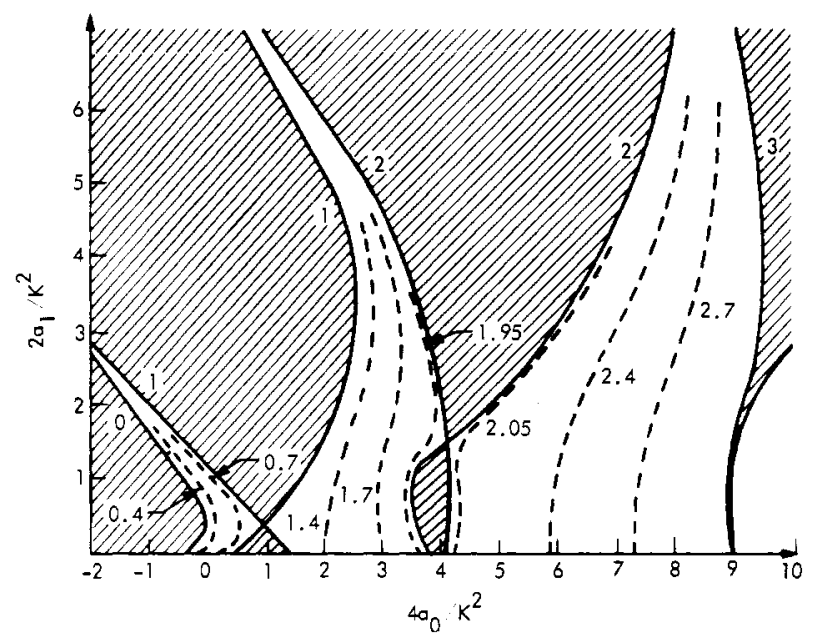

Fig. 3. Stability diagram for the case of TM waves in a sinusoidally periodic dielectric with a relative perturbation $\eta=0.4$ of the dielectric constant. Shaded regions correspond to the regions where the solution for $k$ is complex [35].

wherein the solution for $k$ is pure real. The term "stable" refers to the fact that the corresponding solutions are bounded for any value of $z$. Outside the stable regions, $k$ is complex and its value is

$$
\pm \kappa=m K / 2+i \alpha, \quad m=0,1,2, \ldots
$$

where $(m K / 2)$ is the absolute value of $k$ at the boundaries of the appropriate regions and $\alpha$ is strictly real. These regions are referred to as "unstable regions" because one of the values of $k$ yields a solution which is not bounded at infinity. However, in actual physical situations, this solution is eliminated by the radiation condition.

To illustrate, let us consider the case of an electromagnetic wave in an unbounded sinusoidally periodic medium where the modulation coefficient is $\eta$ and the average dielectric constant is $\epsilon_{0} \epsilon_{r}$. This specifies a line across the diagram (line 2, Fig. 2) which is the locus of the solution $k$ as a function of the frequency $\omega / 2 \pi$ or the unperturbed wavenumber $k$.

We observe that at low frequencies the solution for $k$ is real. As $k$ increases, a certain value is reached where $K$ is equal 
(a) $2 W / A=1 / 10$

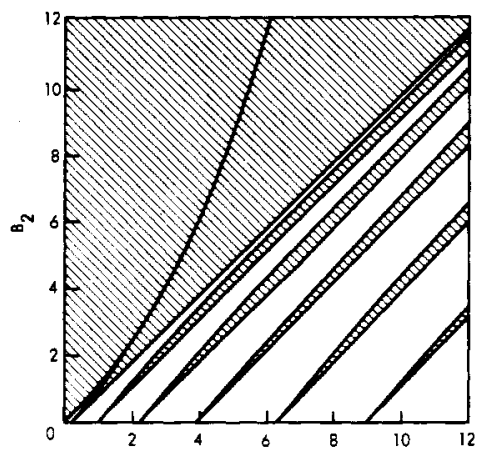

(b) $2 W / A=1.2$

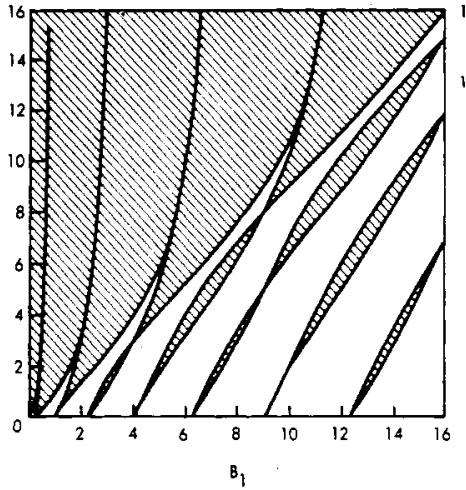

$2 W / A=910$

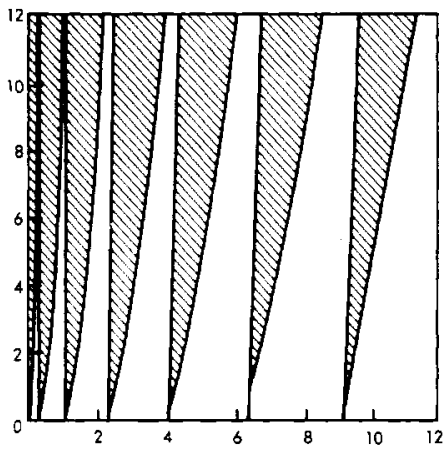

Fig. 4. Regions of real (unshaded) and complex (shaded) solution for the case of a rectangular periodicity for different values of $2 w / \Lambda$ [9].

to $K / 2$. After that, real $(K)$ stays constant while $\alpha$ increases from 0 to $\alpha_{\max }$ then decreases to 0 . This corresponds to the first stopband region. For higher frequencies, the solution crosses the next propagating region until $k=K$, where it crosses into the second stopband, and so on. We remark that the width of the stopband and the value of $\alpha_{\max }$ increase with $\eta$. For $\eta$ small, the stopbands are centered at $k \sqrt{\epsilon_{r}}=m K / 2$ which correspond to the well-known Bragg condition.

The above stability diagram corresponds to the most simple case. When $a_{n} \neq 0$ for all values of $n$, the diagram can exhibit some interesting behavior. Yeh et al. [35] studied the case of TM electromagnetic waves (see Appendix A) where the wave equation reduces to (1) with all $a_{n} \neq 0$. The resulting stability diagram is shown in Fig. 3. It is interesting to remark the crossing of region boundaries which lead to special points where the stopband vanishes for nonzero perturbation. This means that if the medium parameters and the transverse wave vectors are adequately chosen, the wave would have a real wavevector (i.e., no stopband) even if the Bragg condition is satisfied. To illustrate, for the case shown in Fig. 3, if $\gamma K / 2$ is equal to 0.3 where $\gamma^{2}=p^{2}+q^{2}$, then the solution would cross from the first passband to the second passband with no stopband in between.

Another type of periodicity which has been studied extensively is the rectangular periodicity [9], [10], [17], [36], [109]

$$
\begin{aligned}
f(z) & =B_{1}-B_{2} S(z) \\
S(z) & = \begin{cases}0, & \text { for } 0<z<w \\
1, & \text { for } w<z<\Lambda-w \\
0, & \text { for } \Lambda-w<z<\Lambda\end{cases} \\
S(z+\Lambda) & =S(z) .
\end{aligned}
$$

Kronig and Penney [10] considered the case of a delta function potential. Strutt [109] considered the case where the width of the hill and the well are equal, i.e., $w=\Lambda / 4$. Brillouin [17] and Allen [9] considered the general case where $w \neq \Lambda / 4$. The above work was mainly related to the study of the motion of an electron in a crystal with a periodic potential. However, the results are directly applicable to the general case of waves propagating in infinite periodic medium in the direction of the periodicity. Lewis and Hessel [36] extended the previous work to the case in which electromagnetic wave propagation also occurs in a direction transverse to the periodicity. In the case of electronic waves, the dispersion relation ( 8 ) reduces to

$$
\begin{array}{r}
\cos (2 u) \cos (2 v)-\left[\frac{w^{2} u^{2}+(w-\Lambda / 2)^{2} v^{2}}{(\Lambda-2 w) w u v}\right] \sin (2 u) \sin (2 v) \\
=\cos (2 \pi K / K)
\end{array}
$$

where

$$
\begin{aligned}
& u=(\Lambda / 2-w)\left(B_{1}-B_{2}\right)^{1 / 2} \\
& v=w B_{1}^{1 / 2} .
\end{aligned}
$$

In Fig. 4 we show a typical stability diagram for the case of a square potential. A unique feature is the presence of crossing points similar to what happens in the case of the TM waves in a sinusoidal periodicity (crossing points do not exist in the case of Kronig-Penney delta potential model nor in the case of TE waves in a sinusoidal potential). Allen [9] did derive the location of the crossing points to be

$$
\begin{aligned}
& B_{1}=(n \Lambda / 4 w)^{2} \\
& B_{2}=(n \Lambda / 4 w)^{2}-\left[m \Lambda / 4\left(\frac{\Lambda}{2}-w\right)\right]^{2}
\end{aligned}
$$

where $n$ and $m$ are integers with $n \neq 0$ and $B_{2} \neq 0$. Aside from its general mathematical interest, the distribution of these crossing points is connected to the problem of surface states in the study of crystals, as pointed out by Shockley [110].

Another area of investigation was the propagation of electromagnetic waves in a periodic plasma which was studied by Casey et al. [38]. In the case of a TE wave in a sinusoidally periodic plasma, the expression of $D_{n}(9)$ becomes

$$
D_{n}=\frac{2}{\eta}\left[1-\frac{k^{2}-(\kappa+n K)^{2}}{k_{p}^{2}}\right]
$$

where $k_{p}=\omega_{p} / c$ is the plasma wavenumber, and $\omega_{p}$ is the plasma angular frequency, $c$ is the speed of light in vacuum, and $\eta$ is the modulation coefficient. The corresponding stability diagram which is shown in Fig. 2 is still valid except the axes now are

$$
\begin{aligned}
& y=2 \eta\left(\frac{k_{p}}{K}\right)^{2} \\
& x=4\left(k^{2}-k_{p}^{2}\right) / K^{2}
\end{aligned}
$$


so that the stopband and passband characteristics still apply. However, an interesting feature exists in the case of a periodic plasma. We remark that for a fixed value of $\eta, k_{p}$ and $K$ we still can have a real solution for $x$ even if $x<0$ (i.e., $k<k_{p}$ ). This implies that propagation does occur below the plasma frequency, which is not possible in the homogeneous case.

Similar effects occur in the case of TM waves in a periodic plasma; however, Casey et al. [38] did show that the Hill's formulation breaks down at the frequency close to the plasma frequency, because the Fourier series for the function $f(z)$ (see Appendix A) does not converge absolutely.

Another diagram which is commonly used in the study of periodic structures is the Brillouin diagram, which is a graphic representation of the dispersion relation. This diagram allows us to derive, in a relatively simple fashion, most of the properties of closed and open periodic structure. To illustrate, let us consider the case of a wave propagating in a simple homogeneous linear medium. The dispersion relation is

$$
\kappa^{2}=a^{2} k^{2} \quad \text { or } \quad k= \pm a k
$$

where $a$ is a constant which depends on the medium. The corresponding Brillouin diagram is shown in Fig. 5(a). If an infinitesimal periodic perturbation of the propagation medium is introduced, the space harmonics appear. Each one of them will possess a dispersion curve of its own similar to the one in Fig. 5(a), except they will be displaced by $n K$ from each other, as shown in Fig. 5(b). In the infinitesimally perturbed state these space harmonics do not interact with each other.

If the perturbation is increased, strong coupling occurs at the phase-matched points, i.e., intersection points where two space harmonics have the same wave vector or phase velocity. As the two coupled harmonics are contradirectional (group velocities have opposite signs) then a stopband appears. In the passbands, the longitudinal propagation constant $\kappa$ is real when the medium is passive. In the stopbands it is complex as shown in Fig. 5(b). The Brillouin diagram is directly connected to the stability diagram. As a matter of fact, it basically represents the values of $k$ as the solution point moves along the line traced on Fig. 2.

In the above simple example, the Brillouin diagram was used only to derive the nature and location of the coupling. In more complicated cases, such as open radiating structure, the use of the Brillouin diagram is far more rewarding. It allows us to derive the condition for radiation, and the number and direction of radiating beams. This will be discussed in more detail in Section III.

A third diagram which is commonly used, especially in studying source radiation in periodic media is the wave vector diagram. In this case we fix the parameters of the medium and the wave frequency, and plot the transverse wave vector as a function of the longitudinal wave vector (Fig. 6).

The Floquet formulation may be represented by an equivalent electric network. This approach has been used by many authors and is very useful for people who are familiar with the study of electric networks. The coupling between two space harmonics or between a space harmonic and an exciting wave is represented by an ideal transformer with turn ratios equal to the Floquet coefficients. These transformers are connected to transmission lines that are described by the propagation factors $\kappa_{n}$ and characteristic impedances $Z_{n}[128]$.

The space harmonics play a central role in the study of periodic structures and a brief discussion of their properties
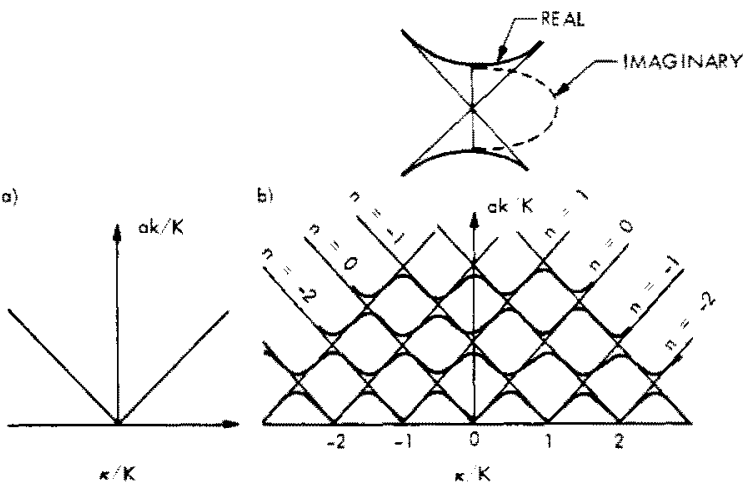

Fig. 5. Brillouin diagram: (a) corresponds to the case of a homogeneous unbounded medium; (b) case of a periodically perturbed unbounded medium. The light lines correspond to the case of infinitely small perturbation. They are obtained from the diagram in (a) by repeating it slong the $k / K$ axis. The heavy lines correspond to finite perturbation. Note that at the intersection points, i.e., Bragg regions, where phase matching occurs, there are gaps where the solution is complex with a fixed real value and a varying imaginary value as shown in the insert in the upper right corner.

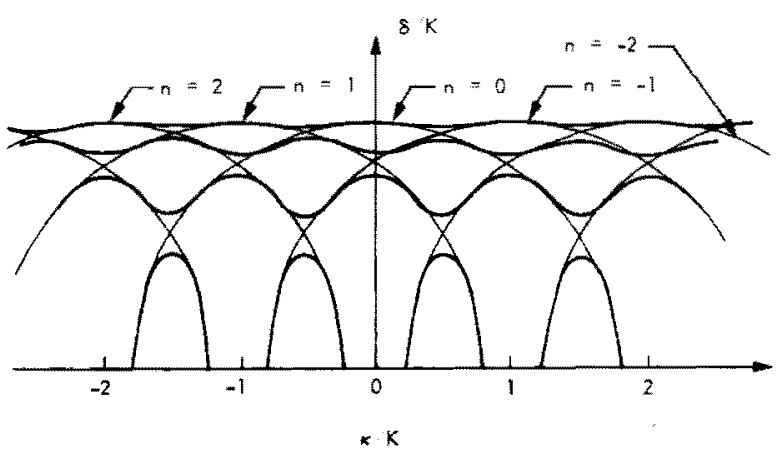

Fig. 6. Wave vector diggram, $\delta$ is the transverse wave vector. The light lines correspond to the case of infinitely small perturbation. They consist of circles centered at $k / K=n$ and radius equal to $\sqrt{\epsilon} k / K$. The heavy lines correspond to the case of finite perturbation.

is in order. As we mentioned previously, the space harmonics do not exist independently. They are portions of a total solution. Each space harmonic has a different wave vector $\kappa_{n}=\kappa+n K$ and a different phase velocity $v_{n}=\omega / \kappa_{n}$. However, all of them have the same group velocity $v_{g}=\partial \omega / \partial \kappa_{n}$ $=\partial \omega / \partial k$. We also remark that the space harmonics that have a large wave vector (i.e., small wavelength) will have proportionally small phase velocity and vice versa such that an observer looking at a specific point in space cannot tell the long fast harmonics from the short slow ones.

The Floquet formulation is relatively simple; however, the numerical calculations are involved. In a large number of cases, the periodic perturbation is relatively small and only few space harmonics have to be taken into account in the neighborhood of a stopband [34]. This approach, called the coupled mode approach, is simple and widely uses [56], [98], [111], [112]. Let us study the case where the wave frequency is very close to the first Bragg frequency; then the propagation wave vector $k$ can be written as

$$
K= \pm(K / 2+\Delta K)
$$

and only the first-order harmonics are nonneglible. Then 
the wave solution can be written as

$$
\begin{aligned}
\psi= & A_{0}^{+} \exp i((K / 2)+\Delta k) z+A_{-1}^{+} \exp [i((K / 2)+\Delta k-K) z] \\
& +A_{0}^{-} \exp i(-(K / 2)-\Delta k) z \\
& +A_{+1}^{-} \exp [i(-(K / 2)-\Delta k+K) z] \\
= & {\left[A_{0}^{+} \exp i \Delta k z+A_{+1}^{-} \exp -i \Delta k z\right] \exp [i(K / 2) z] } \\
& +\left[A_{0}^{-} \exp -i \Delta k z+A_{-1}^{+} \exp i \Delta k z\right] \exp [-i(K / 2) z]
\end{aligned}
$$

which can be rewritten as

$$
\psi=F(z) \exp i(K / 2) z+B(z) \exp [-i(K / 2) z]
$$

This is the form used in the coupled modes approach, i.e., the waves of identical phase velocity are grouped together. Putting the above expression of $\psi(z)$ into the wave equation (1), neglecting the $\partial^{2} F / \partial z^{2}$ and $\partial^{2} B / \partial z^{2}$ terms, and considering the case of sinusoidal periodicity, it is straightforward to find that

$$
\begin{aligned}
& \left(i K \frac{d F}{d z}-\frac{K^{2}}{4} F+a_{0} F+\frac{a_{1}}{2} B\right) \exp [i(K / 2) z] \\
& +\left(-i K \frac{d B}{d z}-\frac{K^{2}}{4} B+a_{0} B+\frac{a_{1}}{2} F\right) \exp [-i(K / 2) z] \\
& +\left(\frac{a_{1}}{2} F \exp i\left(\frac{3}{2}\right) K z+\frac{a_{1}}{2} B \exp \left[-i\left(\frac{3}{2}\right) K z\right]\right)=0 .
\end{aligned}
$$

The last term corresponds to the coupling to second-order harmonics $(n= \pm 2)$ and, therefore, is usually neglected. The above relation has to be satisfied for all values of $z$, thus each term must be equal to zero

$$
\begin{aligned}
& \frac{d F}{d z}+i\left[\frac{K}{4}-\frac{a_{0}}{K}\right] F=i \frac{a_{1}}{K} B \\
& \frac{d B}{d z}-i\left[\frac{K}{4}-\frac{a_{0}}{K}\right] B=-i \frac{a_{1}}{K} F .
\end{aligned}
$$

These are the well-known coupled waves equations, which are usually written in the form

$$
\begin{aligned}
& \frac{d F}{d z}-i \Delta \beta F=i \chi B \\
& \frac{d B}{d z}+i \Delta \beta B=-i \chi F
\end{aligned}
$$

where $\chi=a_{1} / K$ is called the coupling coefficient, and

$$
\Delta \beta=\frac{a_{0}-K^{2} / 4}{K}
$$

is the wave vector mismatch and is equal to $\sqrt{\epsilon_{r}} \Delta k$ where $\Delta k=k_{0}-K / 2$.

The coupled mode approach starts from (21). The solution of (25) is straightforward and is equal to

$$
\begin{aligned}
& F(z)=F_{1} \exp (i \Delta k z)+F_{2} \exp (-i \Delta k z) \\
& B(z)=\frac{\Delta k-\Delta \beta}{\chi} F_{1} \exp (i \Delta k z)-\frac{\Delta k+\Delta \beta}{\chi} F_{2} \exp (-i \Delta k z)
\end{aligned}
$$

where

$$
\Delta k=\sqrt{\Delta \beta^{2}-\chi^{2}} .
$$

The values of $F_{1}$ and $F_{2}$ are determined from the boundary conditions. We note that for $|\Delta \beta|<|\chi|$ the solution for $\Delta k$ is imaginary and corresponds to the imaginary part $\alpha$ of the Flnnuet annroach solution.

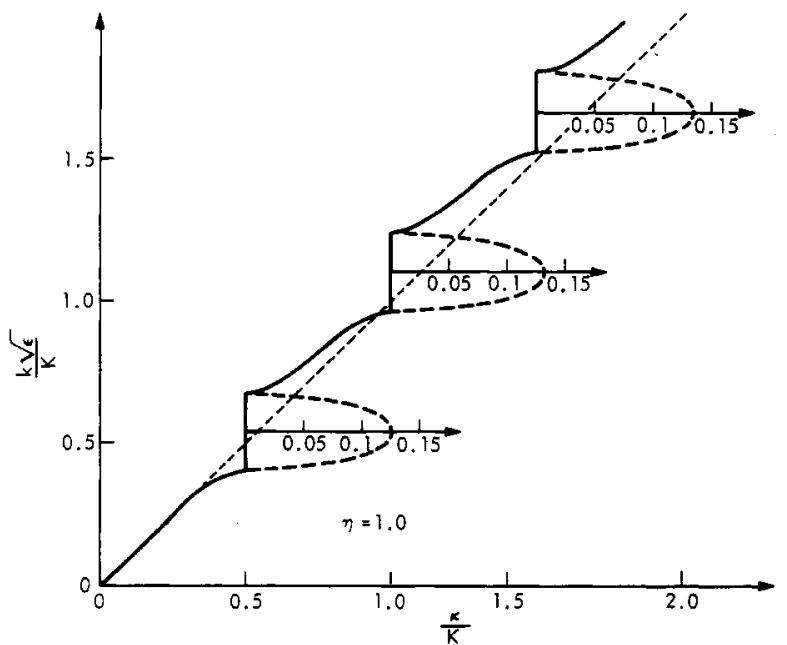

Fig. 7. Brillouin diagram of the first three Bragg interactions for the extreme case of $\eta=1$. The dotted lines are the imaginary part of $\beta / K$. Note the phase speeding for larger values of $k$.

As mentioned above, the coupled wave approach is valid for small $\eta$. However, numerical calculations do show that this approach gives surprisingly good results even for $\eta$ up to 1, which is its maximum value. To illustrate, in Fig. 7 we show the exact dispersion relation using a $19 \times 19$ Floquet matrix for the first, second, and third Bragg regions in the extreme case of $\eta=1$. The stopband region is very similar for all three of them except for the shift above the dispersion line of the unperturbed case. This phase speeding effect can be simply interpreted by computing the average phase velocity across a unit of periodicity. In Fig. 8(a) is presented both the Floquet solution and coupled waves solution. Even for $\eta=1$, the coupled waves theory closely predicts the correct value for the imaginary part of the wave vector; however, it does not predict well the position of the stopband. The coupled wave curve can be used for $\eta$ smaller than 1 by multiplying each scale by $\eta$.

In Fig. 8(b) we show the Floquet solution for $\eta=1,0.1$, and 0.01 . We observe that as $\eta$ decreases, the coupled wave solution becomes a better approximation.

Another point of interest is the number of harmonics needed in the Floquet calculation, i.e., size of the matrix. Fig. 9 does show the results for $3 \times 3,5 \times 5$ and $19 \times 19$ matrices. It is clear that a $5 \times 5$ matrix (up to the third harmonic) is satisfactory with an accuracy better than 1 percent.

The above discussion was mainly concerned with the firstorder Bragg coupling. However, Chu and Tamir [113] derived coupled modes equations, similar to (24), which are valid at higher order Bragg coupling, i.e., when

$$
\sqrt{\epsilon_{r}} k \simeq m K / 2
$$

where $m$ is any positive integer. The main change is in the coupling coefficient $\chi$. For the first-order Bragg, $\chi$ is proportional to the perturbation $\eta$. For the $m$ th order Bragg, Chu and Tamir derived the expression of $\chi$ and showed that it is proportional to

$$
\chi \sim\left(\frac{m}{2^{m} m !}\right)^{2} \eta^{m} .
$$

Su and Gaylord [114] extended the work of Chu and Tamir, and examined higher order diffraction efficiency of thick arbitrary shape diffraction gratings (sinusoidal, square, triangular, and sawtooth shapes).

Recently, Jaggard and Elachi [115] investigated numerically the reqion of validity of the coupled mode approach for higher 


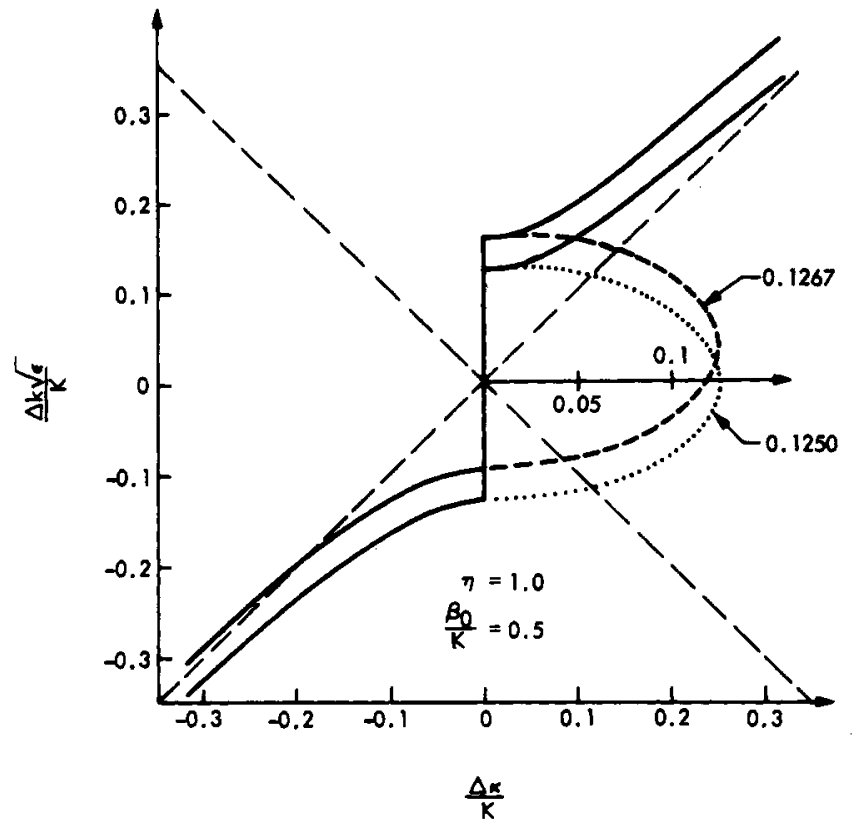

(a)

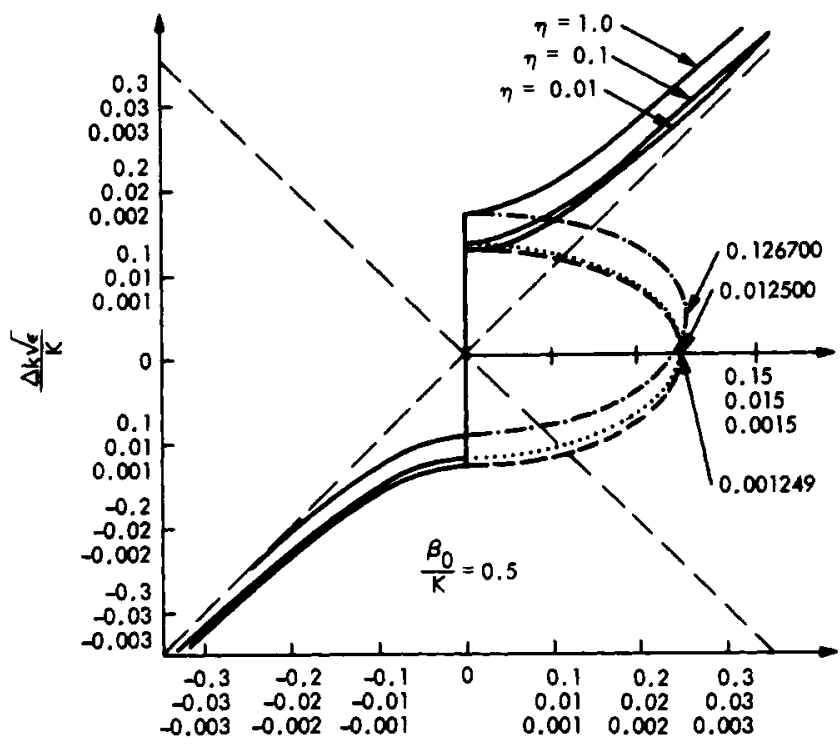

$\frac{\Delta x}{K}$

(b)

Fig. 8. (a) Brillouin diagram of first Bragg interaction with $\eta=1$. This compares Floquet theory (upper dashed curve) with coupled mode theory (lower dotted curve). Dotted and heavily dashed lines are imaginary parts of $\beta / K$. (b) Brillouin diagram of first Bragg interaction for $\eta=1$ (top curve), $\eta=0.1$ (middle curve) and $\eta=0.01$ (bottom curve). Note difference in scales for each case. Imaginary $\beta / K$ are elliptical regions with separate scale.

order interaction. They specifically studied the case of the second-order and third-order Bragg, and computed the dispersion curves using both the exact Floquet formulation and the coupled modes formulation. They showed that the coupled waves equations for the $N$ th order Bragg are

$$
\begin{aligned}
& \frac{d F}{d z}-i \Delta \beta_{N} F=i \chi_{N} B \\
& \frac{d B}{d z}+i \Delta \beta_{N} B=-i \chi_{N} F
\end{aligned}
$$

where the $\Delta \beta_{N}$ and $\chi_{N}$ are functions which depend on $N$.

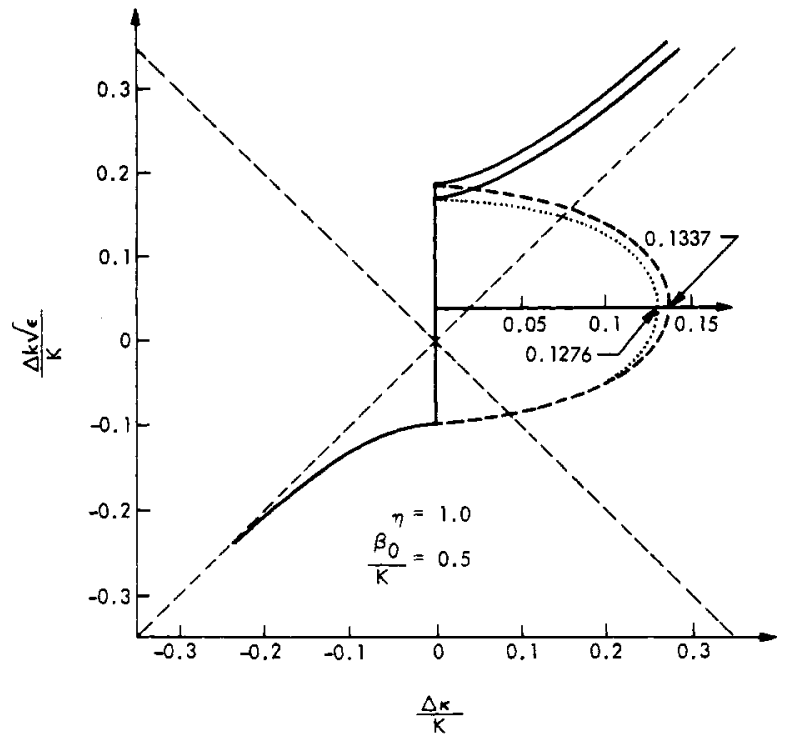

Fig. 9. Brillouin diagram of first Bragg interaction for $\eta=1$ for $3 \times 3$ (upper dashed line) and $5 \times 5$ (lower dotted line) matrix.

A different type of higher order interaction occurs in generally periodic structures. A periodic small perturbation $\Delta \epsilon(z)$ can be written in a Fourier series

$$
\Delta \epsilon(z)=\sum_{n} \eta_{n} \cos (n K z)
$$

Thus Bragg coupling could occur with a higher order Fourier component. For instance, if (27) is satisfied, then there is first-order Bragg coupling with the $m$ th Fourier component. In the case of small perturbations, the results for first-order Bragg coupling are still valid, except that $\eta$ should be replaced by $\eta_{m}$.

In the case of general periodicity, different order Bragg interactions from different Fourier components can add destructively, leading to disappearance of the stopband even if the Bragg condition is satisfied. This effect was analyzed using the Floquet and coupled waves approach [115].

An important aspect of the above formulations (Floquet and coupled wave) is that there was no requirement on the nature of $\epsilon$ or $\eta$. Thus they are valid for active and passive media, and for perturbation of the real or imaginary part of the dielectric constant. In the case of an active medium of gain coefficient $g$, the coupled waves equations become

$$
\begin{aligned}
& \frac{d F}{d z}-(g+i \Delta \beta) F=i \chi B \\
& \frac{d B}{d z}+(g+i \Delta \beta) B=-i \chi F .
\end{aligned}
$$

In the case of gain coupling (i.e., gain periodicity) $\chi=i \chi$ and $\Delta K=\sqrt{\Delta \beta^{2}+|\chi|^{2}}$. Thus there is no stopband. However, distributed feedback still occurs because of the change in the propagation wave vector.

The behavior of the effective gain or loss near the Bragg region is shown in Fig. 10 for the case of an active, passive or lossy periodic medium. In the case of a passive medium, a stopband exists. If the medium is lossy, the loss coefficient increases in the Bragg region. A detailed numerical calculation of this effect was conducted by Minakovic and Gokgor [116] who showed that the attenuation in a periodically loaded circular waveguide can be considerably higher than when the guide is completely filled. If the medium is slightly active then the gain is drastically increased in the Brase region as a result 


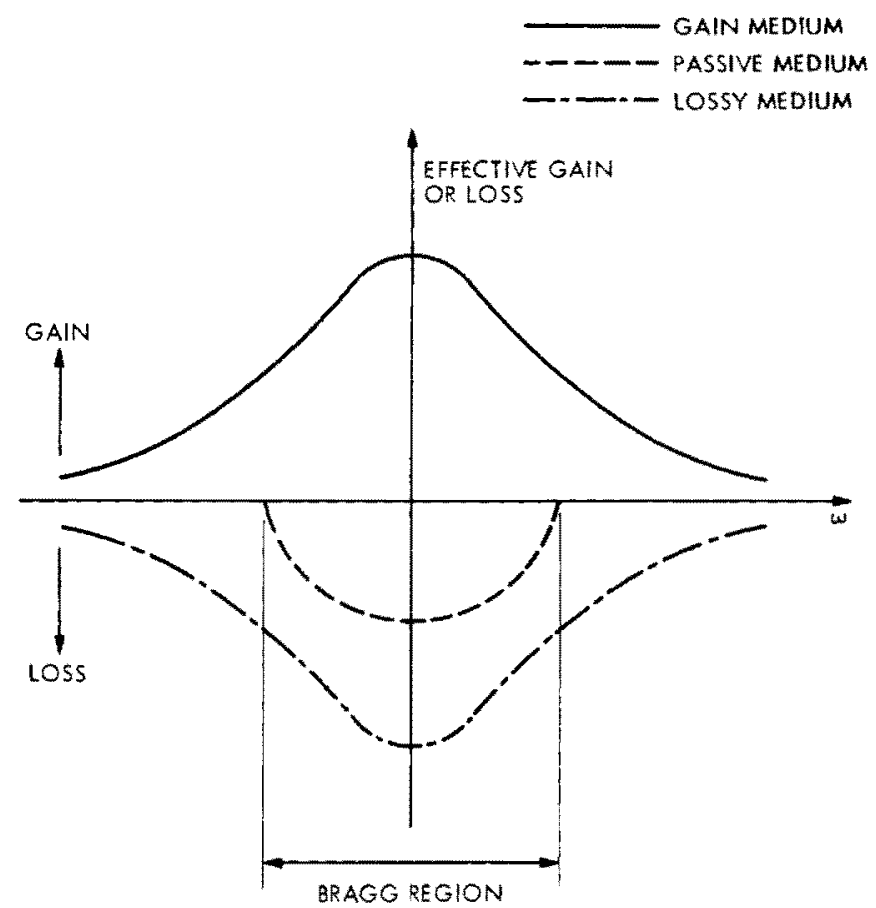

Fig. 10. Effective gain or loss near the Bragg region in the case of an active, passive or lossy periodic medium.

of a periodic change in the real index of refraction. The increase in the effective gain or loss can somehow be explained by the fact that the periodicity bounces the wave back and forth, and therefore increases its effective line path per unit length of the medium. If the periodicity is in the gain coefficient, the effective gain is smaller than the average gain [77].

A somewhat different approach is commoniy used to study optical multilayers $[15],[16]$ and is very useful for the study of lumped structures. It simply consists of the derivation of a characteristic matrix of one unit cell which correspond to one period. This matrix relates the two components of the electric (or magnetic) vectors in the input plane, to the components at the output plane of that cell. Thus if we have a periodically stratified medium which consists of $N$ periods, the total characteristic matrix $T_{T}$ is simply equal to

$$
n_{T}=\underbrace{n \cdot \ldots n}_{N \text { times }}=n^{N} \text {. }
$$

To evaluate the elements of the matrix $N_{T}$, a result from the theory of unimodular matrices is used [15]. If

$$
n=\left[\begin{array}{ll}
m_{11} & m_{12} \\
m_{21} & m_{22}
\end{array}\right] \text {. }
$$

Then

$$
R_{T}=\left[\begin{array}{ll}
m_{11} U_{N-1}(a)-U_{N-2}(a) & m_{12} U_{N-1}(a) \\
m_{21} U_{N-1}(a) & m_{22} U_{N-1}(a)-U_{N-2}(a)
\end{array}\right]
$$

where $a=\left(m_{11}+m_{22}\right) / 2$ and $U_{N}$ are the Chebyshev polynomials of the second kind:

$$
U_{N}(x)=\frac{\sin \left\lfloor(N+1) \cos ^{-1}(x)\right\rfloor}{\sqrt{1-x^{2}}}
$$

Such an approach has the nice advantage that it can be simply applied to any value of $N$. However, it requires that the matrix $M$ is known or can be simply derived. This is the case in the study of optical multilayers which consist of successions of homogeneous layers or in the study of periodic electric filters.

A somewhat similar but more complicated and general approach was given by Mead [117] who studied wave propagation in linear periodic systems with multiple coupling. Mead's paper was a generalization of the previous work conducted in the field of structural engineering as related to the propagation of vibrational and flexural waves on periodic systems of beams and plates. Examples of such systems are: tall apartment blocks having a uniform structure and identical stories; aeroplane fuselage structure consisting of a uniform shell reinforced at regular intervals by an orthogonal set of identical stiffeners. The approach basically consists in deriving the characteristic matrix which relates the output parameters to the input parameters of a unit element of the structure. It also adopts itself nicely to the study of two-dimensional lumped periodic structures. To illustrate, let us consider the simple case of natural flexural waves propagating on an infinite periodically supported beams and plates which was studied by Sen Gupta [118]. The slopes $\theta$ and end-moments $M$ at the input and output of a plate are related by

$$
\begin{gathered}
\theta_{i}=\alpha_{11} M_{i}+\alpha_{12} M_{0} \\
\theta_{0}=-\alpha_{12} M_{i}-\alpha_{11} M_{0} .
\end{gathered}
$$

For a flexural wave of propagation constant $k$ we have

$$
M_{0}=M_{i} e^{-k} \quad \theta_{0}=\theta_{i} e^{-k}
$$

which give

$$
\cosh k=-\alpha_{11} / \alpha_{12}
$$

the elements $\alpha_{11}$ and $\alpha_{12}$ are functions of the frequency and can be derived by solving the free vibration equation of a plate or a beam.

\section{Waves in a Bounded Periodic Structure}

The presence of boundaries adds a new dimension to the analysis of periodic structures. In this section we will first consider the case of closed structures where the transverse boundaries are completely reflecting. Then we will briefly review the extensive work in the field of periodic open structures where bounded and radiating modes can be present. Finally, we will discuss the case of waves incident on a periodic half-space, or slab, where the periodicity is parallel or perpendicular to the boundaries.

When the longitudinally periodic medium has complete reflecting transverse boundaries, the transverse wave numbers are fixed exclusively by the geometry. In the case of a rectangular waveguide of dimensions $h_{1}$ and $h_{2}$, the transverse wavenumbers are

$$
\begin{aligned}
s \text { or } p & =m \pi / h_{1} \\
w \text { or } q & =m \pi / h_{2}
\end{aligned}
$$

where $m$ and $r$ are integers. These expressions of $p, q, s$, and $w$ are then used in the expressions of $f(z)$ given in Appendix A.

Tamir et al. [34] and Yeh et al. [35] did consider the case of waveguide excitation in their work on the propagation of TE and TM electromagnetic waves in sinusoidally periodic media. The longitudinal propagation wave vector can be easily derived from the stability diagram in Figs. 2 and 3. For a spe- 
cific structure and a specific mode (i.e., specific transverse wave vector) the locus of the solution is a straight line, as shown in Fig. 2. This line is characterized by its slope

$$
\text { slope }=a_{1} / 2 k^{2}
$$

and its origin, which corresponds to $a_{1}=0$ and $a_{0}=-\left(s^{2}+p^{2}\right)$. We observe that as $k$ increases, the solution for $k$ passes through bands of real solutions and complex solutions as in the case of unbounded media. One unique feature is that propagation occurs at some frequencies below the cutoff frequency of the homogeneous guide. This effect occurs for all the waveguide modes and is similar to what occurs in the case of a periodic plasma.

If the transverse boundaries are not completely reflecting, things get far more complicated and more interesting. That results mainly from the possibility of energy leakage. Open periodic structures support surface waves which travel parallel to the structure, and leaky waves which are guided by the structure but radiate or leak energy continuously into the exterior regions. Both types of waves appear as characteristic solutions of the boundary-value problem prescribed by the waveguide configuration.

Open periodic structures are encountered in current engineering practice in a variety of applications, such as travelingwave slot or dipole arrays, log periodic antennas, and periodically modulated slow wave antennas. The theoretical analysis of these radiating structures was mainly based on the simple model of periodically modulated reactance surface. A review of the work in this field is given by Hessel [33]. In this section, we will discuss only the use of the Brillouin diagram which gives, very simply and elegantly, most of the properties of open periodic structures.

Another field of application of open periodic structures that has attracted considerable interest in the last few years is the new field of integrated optics. Thin-film periodic structures have been used in such applications as beam-to-surface-wave couplers, filters, distributed feedback amplifiers and lasers, nonlinear generation of second harmonics, and beam deflection or steering devices of the Bragg type. The periodicity can be a perturbation in the dielectric constant of the guiding film or its substrate, or a perturbation of the boundary. The analysis of these structures has been considered in the context of several specific structures using perturbation techniques [70], [90], [98], [111] or rigorous analysis [119].

To simplify matters, planar geometry will be assumed, with the $x=0$ plane as the interface between the guiding region and exterior region $(x>0)$. The longitudinal wave number of the nth space harmonic in both regions is equal to $K+n K$ and the transverse wave number $\delta_{n}$ in the exterior region is

$$
\delta_{n}^{2}=k^{2}-(K+n K)^{2} .
$$

If $n$ is such that $(\kappa+n K)^{2}>k^{2}$, as will be the case for most of the space harmonics at any given frequency, then $\delta_{n}$ is imaginary and the space harmonic is a slow wave. If some space harmonics are present for which $(k+n K)^{2}<k^{2}$, then $\delta_{n}$ values are real and these space harmonics are fast waves. If we consider the Brillouin diagram (Fig. 11), then we can divide it in slow wave or bound region and fast wave or unbound region. If the operating point of one harmonic is inside a triangle, then all other space harmonic solutions also lie within the sequence of triangles. Thus, all the space harmonics, and the total solution, are bound to the interface. However, if the solution is outside the triangles, then at least one of the space harmonics is in the radiation region of the homogeneous struc-

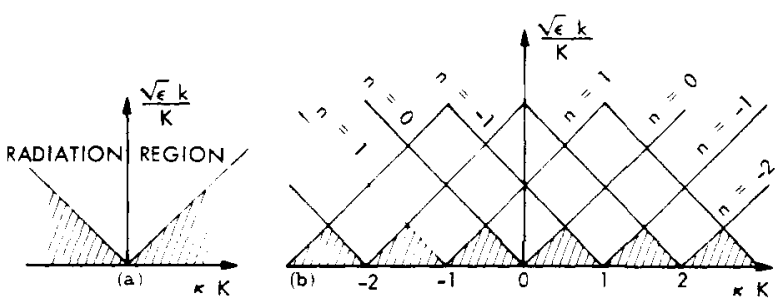

Fig. 11. Brillouin diagram. (a) Showing the radiation region for a homogeneous structure. (b) Showing a periodic structure. The unshaded region corresponds to where at least one space harmonic is radiating. The shaded triangles correspond to the region where all space harmonics and therefore to total solution are bounded.

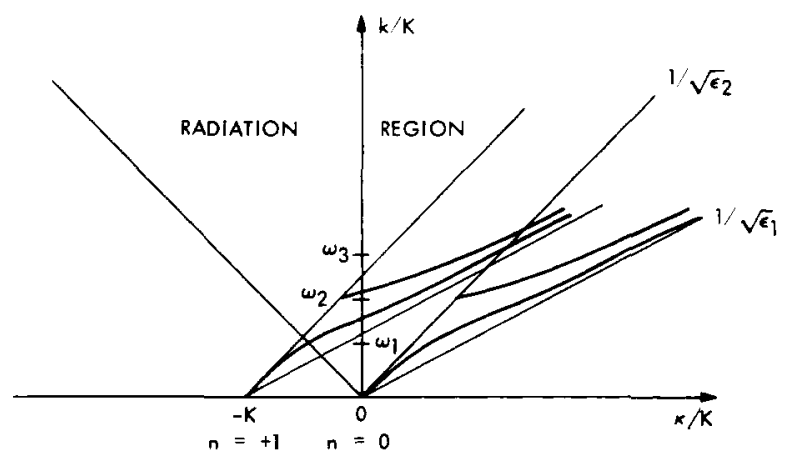

Fig. 12. Brillouin diagram for a periodic slab showing the $n=0$ and $n=$ 1 harmonics. Only the two first waveguide modes are shown. At $w=$ $\omega_{1}$ the $n=1$ harmonic of the first mode crosses into the radiation mode leading to a backfire beam. As the frequency increases, the beam swings around to become an end fire beam at $\omega_{3}$ and then disappears. Meanwhile at $\omega=\omega_{2}$ the radiation beam of the second mode appears.

ture, and the total wave is no longer completely bound. The radiating harmonics are leaky waves which extract energy from the total wave along the structure.

Another property of open periodic structures, which can be easily determined from the Brillouin diagram is the number of radiating beams and their angle of radiation. The beam angle $\theta_{n}$ from broadside is equal to

$$
\sin \theta_{n}=(K+n K) / k \text {. }
$$

Thus, the number of radiating beams at any frequency is equal to the number of space harmonic solutions lying in the radiation region at that value of $k d$ (i.e., points such that $|k+n K|<k)$. Basically each angle corresponds to one of the radiating space harmonics.

To illustrate, let us consider the simple case of a dielectric slab of relative dielectric constant $\epsilon_{1}+\eta \epsilon_{1} \cos K z$ imbedded in vacuum (i.e., dielectric constant $=1$ ). The corresponding Brillouin diagram is shown in Fig. 12. Only the first two waveguide modes are sketched. We observed that as the frequency $\omega$ is increased from zero, no radiation beams are present. For $\omega=\omega_{1}$ a backfire beam appears which corresponds to the first harmonic of the basic mode. As the frequency increases, the beam angle changes gradually from backfire to end fire at $\omega=\omega_{3}$, then diappears. In the interim, at $\omega=\omega_{2}$ the beam from the first harmonic of the first mode appears. Depending on the values of $\epsilon_{1}$ and $K$, it is possible that the beam from the second or higher harmonic of the second mode appears before the one corresponding to the first harmonic disappears. Thus depending on the choice of $\epsilon_{1}, K$, and the excited modes, a large number of beams can be present, at different angles, for any operating frequency above $\omega_{1}$. 


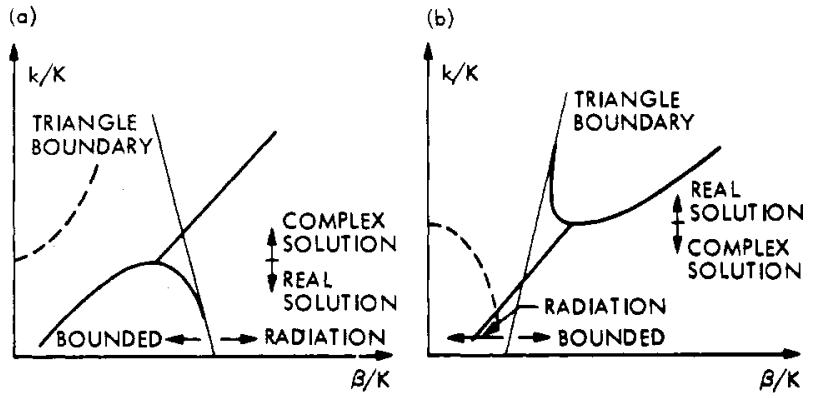

Fig. 13. Sketches of the Brillouin diagram, i.e., wave vector solution near the boundaries of the radiation triangle (see [33] for details). The light line is the boundary of the radiation triangle (see Fig. 11). The continuous line is the real part of $\beta$. The dashed line is the imaginary part of $\beta$. (a) and (b) correspond to contradirectionsl or codirectional crossing.

An effect which cannot be predicted from the Brillouin diagram is the coupling which occurs at the crossing of the dispersion curve with one of the sides of a triangle. This corresponds to the interaction between a particular space harmonic and a plane wave skimming along the surface. In antenna theory, if the dispersion curve and the triangle boundary line are codirectional, the intersection region is called end-fire region. If they are contradirectional, it is called back-fire region. Hessel and Oliner [120], Wang [121], Oliner [122], and Hessel [33] analyzed these regions in detail. They showed that the complex solution outside the triangles do extend slightly inside the triangle region where, close to the boundary, the real solution is double valued (Fig. 13). These regions correspond to the Wood anomalies regions which will be discussed later in this section.

The Brillouin diagram gives insight in many properties of periodic structures in a very simple and elegant way. However, a complete quantitative evaluation of the field in these structures requires the solution of the wave equation. In the case of periodic antennas, a simple model of a periodic surface reactance have been extensively and rigorously analyzed [33]. In the case of thin films with periodic modulation of the dielectric constant, most of the work used perturbation techniques which are valid for small modulations [70], [90], [111], [123]-[127].

Let us assume a $p$ th mode wave $u_{p}(x) e^{i k_{p} z}$ propagating in a homogeneous thin-film waveguide of thickness $2 W$ and dielectric constant $\epsilon$.

If a small perturbation $\eta \epsilon \cos (K z)$ is added, then a convection current $J_{c}$ is generated inside the guide

$$
\begin{aligned}
J_{c} & =\Delta \epsilon \frac{\partial E}{\partial t}=-i \omega \epsilon \eta \cos (K z) u_{p}(x) \exp \left(i \kappa_{p} z\right) \\
& =-i \frac{\omega \epsilon}{2} \eta u_{p}(x)\left[\exp \left[i\left(k_{p}+K\right) z\right]+\exp \left[i\left(\kappa_{p}-K\right) z\right]\right]
\end{aligned}
$$$$
|x|<W . \quad(40)
$$

This current has two components with different longitudinal wave vectors. If any one of the wave vectors is equal to the wave vector $\pm k_{q}$ of the $q$ th mode, then phase-matched coupling and energy transfer occurs between these modes.

The coupling could be of the codirectional or contradirectional type. The first element $e^{i\left(\kappa_{p}+K\right) z}$ can achieve only codirectional coupling. The second element $e^{i\left(\kappa_{p}-K\right) z}$ can achieve both couplings depending on the relative values of $k_{p}$ and $K$. Once the longitudinal phase matching is satisfied, only the part of the convection current which is also transversely phase matched would play a role in the coupling. The transverse function $u_{p}(x) h(x)$ where $h(x)=1$ for $|x|<W$ and 0 otherwise, can be expanded in terms of the unperturbed modes of the guide, i.e.,

$$
u_{p}(x) h(x)=\sum c_{p j} u_{j}(x)
$$

where

$$
c_{p j}=\frac{\int_{-\infty}^{+\infty} u_{p}(x) h(x) u_{j}^{*}(x) d x}{\int_{-\infty}^{+\infty} u_{j}(x) u_{j}^{*}(x) d x}
$$

$$
=\frac{\int_{-w}^{+w} u_{p}(x) u_{j}^{*}(x) d x}{\int_{-\infty}^{+\infty} u_{j}(x) u_{j}^{*}(x) d x} .
$$

Thus if the $p$ th mode is longitudinally phase matched to the qth mode, the part of the convection current which achieves the energy coupling is equal to

$$
J_{c p q}=-i \frac{\omega}{2} \eta \in C_{p q} u_{q}(x)
$$

The term $c_{p q}$ is what is known as the overlap integral. Once the $q$ th mode is excited it also couples to the $p$ th mode and vice versa. The behavior of the coupling coefficient for a variety of structures has been analyzed by Elachi et al. in a number of papers [70], [90], [123], [124].

A similar approach can be used to correlate the coupling between a guided mode and a radiation mode. This work was conducted in detail by Marcuse [111], [125] for the case of a surface corrugation or a thin layer of an inhomogeneous medium around the boundary of the guide. He derived the power loss per unit length and the pattern of the radiation beam. An interesting result is that the energy loss to one guided mode is proportional to $L^{2}$, while the loss to the radiation modes is proportional to $L$, where $L$ is the interaction length.

The rigorous solution for a periodically modulated slab is more involved. Recently, Peng et al. [119] formulated the exact solution which is valid for any volume or surface periodicity, and resolved it numerically for the cases of holographic layers and for rectangularly corrugated gratings. A brief review of their formulation follows.

The wave equation for the field vectors of TE and TM waves can be reduced to the scalar equation (see Appendix A)

$$
\nabla^{2} \psi+f(z) \psi=0
$$

where $f(z)$ can be written as

$$
f(z)=\sum_{n} a_{n} \cos (n K z)
$$

and the coefficient $a_{n}$ are known for a given grating and frequency. The solution for $\psi$ may be written as

$$
\psi=\sum_{n} A_{n}(x) \exp i(K+n K) z .
$$

Inserting this representation in (44), we obtain

$$
\frac{d^{2} \mathbb{Q}}{d x^{2}}=-P Q
$$


where $\mathrm{Q}$ is a column vector with elements $A_{n}(x)$ and $P$ is a constant matrix (independent of $x$ ) whose elements are defined by

$$
P_{n e}=k^{2} a_{n-e}-(K+n K)^{2} \Delta_{n e}
$$

where $\Delta_{n e}$ is Kronecker's delta function. The solution of the above system of equations is of the form

$$
Q=\mathcal{E} \exp (i \delta x)
$$

where $\delta$ is the transverse propagation constant and $\mathcal{C}$ is a constant vector. Substituting into (47), we obtain the system of linear equations:

$$
\left[P-\delta^{2} I\right] \mathcal{C}=0 .
$$

Where $I$ is a unit matrix of infinite order. The characteristic equation of the above system, i.e.:

$$
\operatorname{det}\left[P-\delta^{2} I\right]=0
$$

gives the eigenvalues $\delta_{m}^{2}$, and the corresponding eigenvectors $C_{m}^{\prime}$ (with elements $C_{m n}$ ) can then be obtained by solving (50). Thus the solution for the field can be written in the form

$$
\begin{aligned}
\psi=\sum_{m}\left[A_{m}^{+} \exp i \delta_{m} x+A_{m}^{-} \exp -i \delta_{m} x\right] \\
\cdot \sum_{n} C_{m n} \exp i(\kappa+n K) z .
\end{aligned}
$$

This shows that the solution is a summation over an infinite number of modes, each of which consists of an infinite number of space harmonics. For a given $\epsilon(z)$ and wave frequency, the unknowns in the above expression are $k$ and $A_{m}^{ \pm}$. The values of $\delta_{m}$ and $C_{m n}$ are determined from (50) and (51). The values of $A_{m}^{ \pm}$and $\kappa$ can be determined from the boundary conditions and the excitation condition. Peng et al. [119] derived the dispersion equation in a matrix form, and studied the case of guided waves and waves excited by a beam incident from the outside of the slab. They also studied exactly the case of multilayered periodic structures. They solved numerically for the case of a periodic slab on a substrate and derived the propagation wave vector (and attenuation constant $\alpha$ ) in the bounded and radiation regions for both TE and TM modes. They showed that $\alpha$ varies slowly with frequency in the leaky wave region (radiation region) except in the vicinity of Wood's anomalies, which correspond to the onset of additional leaky wave beams in the air or substrate regions. For the TM waves, additional nulls for $\alpha$ appear which are due to a Brewster angle phenomenon for higher harmonics inside the grating layer.

It is interesting to point out that many harmonics are such that

$$
(k+n K)^{2}>\epsilon_{1} k^{2} .
$$

This implies that most of the space harmonics are evanescent both inside and outside the guide, i.e., they exist mainly in the neighborhood of the boundary. However, it should be emphasized again that individual space harmonics cannot exist on their own, and if only a few harmonics are leaky, then the whole wave loses energy because of the crosscoupling.

Even though most of the work was directed toward the case of thin films, the techniques can be used to study more complicated structures. Marcuse considered the case of hollow slabs [62]. Elachi et al. [90], [124] analyzed the case of periodic fiber guides and diffuse guides as associated with their application to DFB laser using the perturbation technique. In
Peng et al. [119], their exact solution could be generalized to cover these and other geometries.

Up to now we considered the use of open and closed guiding structures, where the wave in the periodic medium propagates along the periodicity axis. Another type of bounded structure that has been studied corresponds to the case of waves incident, from the outside, on a slab or half-space which is periodically modulated parallel or perpendicular to the boundary. Tamir and Wang [37], and Tamir [128], analyzed rigorously and in detail the scattering of electromagnetic waves by sinusoidally stratified half-space, where the direction of the periodicity is parallel to the air-dielectric interface. Of special interest was their analysis of the anomalies that appear in gratings. When the wavelength $\lambda$ or the incident angle $\theta$ of the incident plane wave are slowly varying, it is expected that the amplitudes of the various modes would likewise vary slowly. It turns out, however, that rapid fluctuations occur in some of the modal amplitudes at the Rayleigh wavelengths

$$
\lambda_{R}=\frac{\Lambda}{m}(1 \pm \sin \theta)
$$

and at the Bragg wavelengths

$$
\lambda_{B}=\frac{2 \Lambda}{m} \sin \theta
$$

where $m$ is a positive integer. In the air region, the rapid fluctuations appear only at the Rayleigh wavelengths and they correspond to Wood anomalies [129] which are characteristic of reflection gratings. These anomalies occur whenever a reflected mode reaches grazing incidence (cutoff) or a mode in the modulated dielectric region reaches grazing incidence. The wavelength which corresponds to the last case cannot be expressed in simple terms except when the periodic modulation is small, in that case

$$
\lambda_{R^{\prime}}=\frac{2 \Lambda}{m}\left(\epsilon_{r}^{1 / 2} \pm \sin \theta\right)
$$

where $\epsilon_{r}$ is the relative average permittivity of the dielectric. These Wood anomalies basically correspond to the coupling between an oblique wave and a wave propagating along the surface. An intensive analysis and review of the work on Wood's anomalies is given by Hessel and Oliner [129].

In the modulated medium itself, the fluctuations due to the Wood anomalies are usually obscured by the strong interference effect which peaks at the Bragg wavelengths, and is due to the periodicity of the medium. This resonance behavior results in a standing wave pattern produced by two modes possessing nearly equal amplitudes but different wave numbers. In that respect, the resonance process is closely analogous to the Borrman effect [130], [131] which occurs as an anomalous transmission of $\mathrm{X}$-rays through thick perfect crystals, when the incidence is at the Bragg angles. However, even though the periodicity is in one dimension only, the resonant effect in the modulated half-space results in a standing-wave pattern with respect to both the $z$ and $x$ directions with wavelengths of $2 \Lambda$ and $2 \Lambda_{m}^{\prime}$, respectively, where $\Lambda_{m}^{\prime}$ is strongly dependent on the modulation of the medium such that $\Lambda_{m}^{\prime} \gg \lambda$ if the modulation is small. Thus, this resonant behavior produces a repetitive pattern of oblong rectangular cells and is denoted by the term "cell resonance." The periodicity in the field along the $x$ direction can be explained by simple physical reasoning. When the incident plane wave crosses the interface of the modulated dielectric, it starts traveling initially as if no 


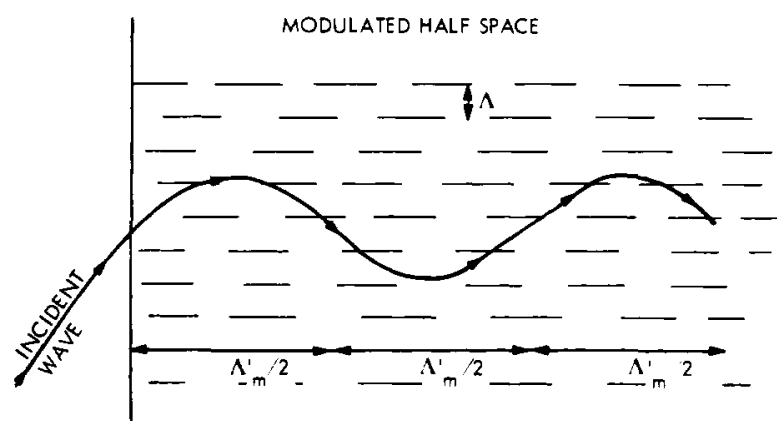

Fig. 14. Ray trajectory in a transversely modulated half-space.
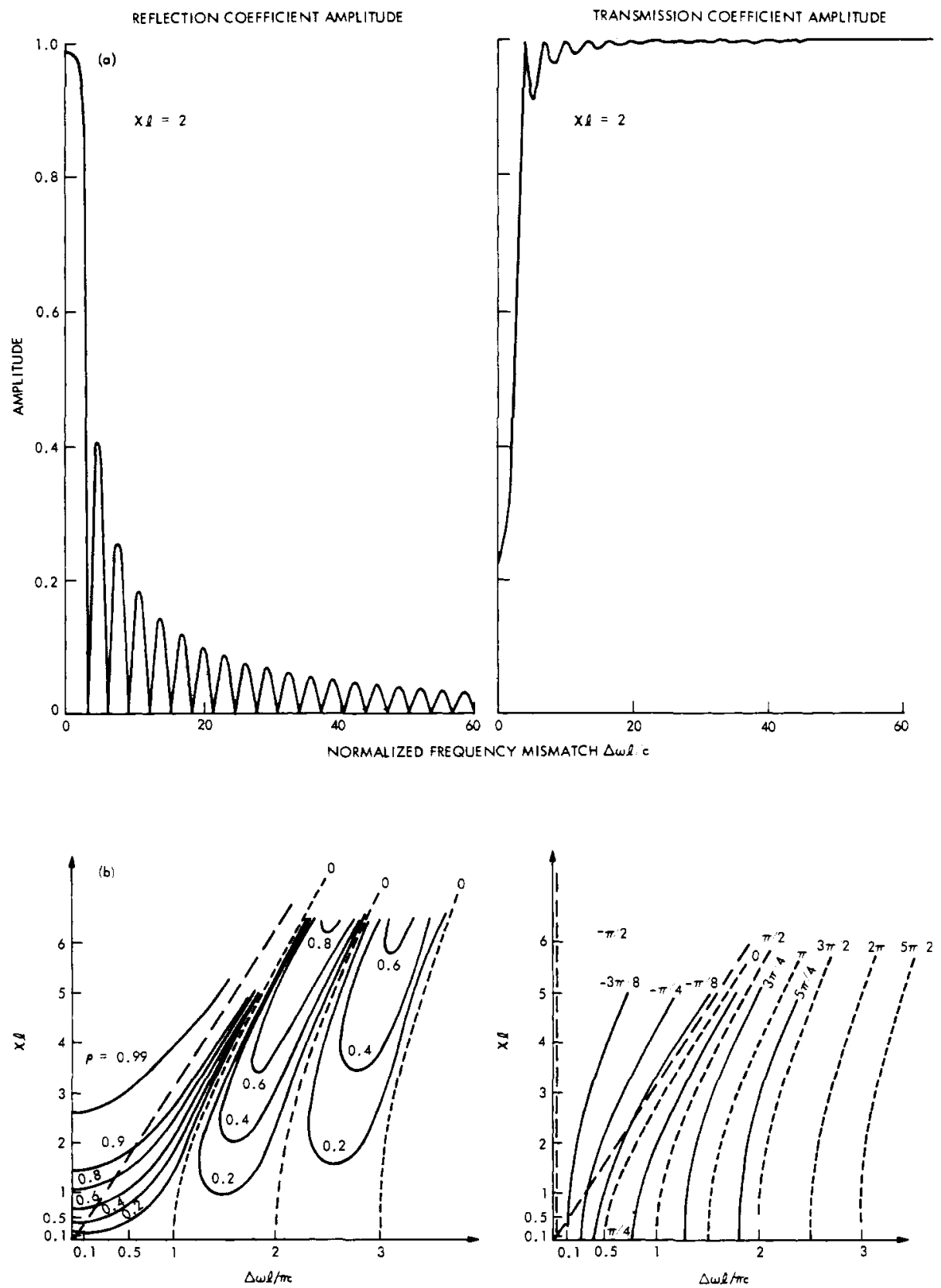

Fig. 15. (a) Magnitude of reflection and transmission coefficients as function of normalized frequency mismatch $\Delta \omega l / c$ for fixed coupling: $x l=2$. (b) Equimagnitude and equiphase curves of reflection coefficient as function $\Delta \omega l / \pi c$ and $x l$. Reflection curve in Fig. 15(a) corresponds to horizontal line $(X I=2)$ across graph. 
periodicity were present. However, as the Bragg condition is satisfied, the successive reflections from the periodic perturbations add constructively, and more and more power propagates in the negative $z$ direction. After a certain time, most of the energy is propagating toward negative $z$. However, as the periodic perturbation reflects irrespective of the wave direction, more and more energy is reflected back in the positive direction, and so on. This results in an oscillatory power flow line as shown in Fig. 14. Evidently, the larger the modulation, the stronger are the reflections at each perturbation, the faster the energy flow is reversed, which implies a smaller value of $\Lambda_{m}^{\prime}$. Inversely, if the modulation vanishes, the length $\Lambda_{m}^{\prime} \rightarrow \infty$. This effect is analyzed in detail by Tamir [128] .

In the case where the periodicity direction is perpendicular to the boundary, the analysis is far more simple. The transverse wave number is fixed by the incident or source wave, and the problem becomes somewhat similar to the closed bounded structures. This problem was specifically studied by Tamir et al. [34] for TE waves and Yeh et al. [35] for TM waves.

Finally, we briefly review the case of a slab of thickness $2 l$ with small perturbation in the direction perpendicular to the boundaries. The importance of this problem is mainly in its application to the study of DFB laser structures that we will review later. Starting from the coupled modes equations (Section II) and taking the adequate boundary conditions [132], the reflection coefficient $R$ and transmission coefficient $T$ can be derived in a straightforward way as

$$
\begin{aligned}
& R=\frac{i \chi}{\left[\sqrt{\chi^{2}-\Delta \beta^{2}} \operatorname{coth}\left(l \sqrt{\chi^{2}-\Delta \beta^{2}}\right)-i \Delta \beta\right]} \\
& T=\frac{\sqrt{\chi^{2}-\Delta \beta^{2}}}{\sqrt{\chi^{2}-\Delta \beta^{2}} \cosh \left(l \sqrt{\chi^{2}-\Delta \beta^{2}}\right)-i \Delta \beta \sinh \left(l \sqrt{\chi^{2}-\Delta \beta^{2}}\right)} .
\end{aligned}
$$

Where we have assumed that there is no Fresnel-type reflections at the end of the structure, the thickness of the periodic slab is $l$. In Fig. 15 are shown the magnitudes of the reflection and transmission coefficients as a function of the mismatch $\Delta \beta l$ for a fixed value of the coupling coefficient $\chi l$. The properties of this structure are identical to an electric circuit filter.

\section{BOUNDARY PERIOdICITY}

Bragg scattering and/or distributed feedback also results from boundary periodicity. The presence of space harmonics is required to satisfy the boundary conditions. The scattering of a scalar wave incident on an infinite corrugated interface separating two different homogeneous media were studied about seventy years ago by Lord Rayleigh [133]. However, the Rayleigh method of solution has aroused some controversy. If the plane wave is assumed to fall on the interface from above, then, in the region above the highest points on the surface, it is generally accepted that the scattered field may be represented by a linear combination of space harmonics, each of which propagates, or is attenuated, away from the surface. The controversy has its origin in the further assumption (admittedly unjustified by Rayleigh) that this representation is also valid within the corrugation and on the surface itself; henceforth, this will be termed the Rayleigh assumption. It has been shown [134]-[139] that this assumption may result in serious errors if the periodic variation is not sufficiently small. Petit and Cadilhac [134]-[137], and Millar [138] did finally show that, in the case of a sinusoidal profile
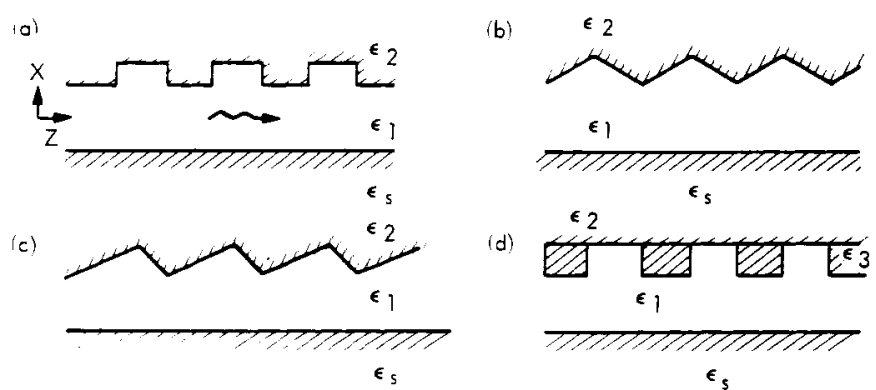

Fig. 16. Different configurations of guides with periodic surface which have been considered in the literature.

of the form $h \cos (K z)$, the Rayleigh assumption is valid for $K h<0.448$ and could not be valid for $K h>0.448$. Thus the Rayleigh assumption yields good results only if the periodic change is sufficiently small, so that its use may produce erroneous results in some practical cases, such as thick corrugated grating which have groove depths comparable to the wavelength. Another approximation has been the use of a Rayleigh assumption which incorrectly neglects the presence of incoming waves in the grating region [126], [140], [141].

Neviere et al. [135], [136] and Peng et al. [119], [139] resolved rigorously the problem of electromagnetic waves in a dielectric waveguide with a general corrugated boundary. The approach taken by Neviere et al. requires a numerical integration. They studied the scattering of a plane TE or TM wave incident on a grating which is underlain by a multilayered halfspace. They also analyzed the case of limited incident beam and of guided waves. Peng et al. derived the solution in a form that lends itself readily to physical interpretation in terms of the effects due to the individual partial (space harmonics) fields.

In the case where the grating profile is a rectangular profile (Fig. 16), the grating could be replaced by a layer of constant thickness but modulated dielectric constant. Then the problem reduces to the cases discussed in Section III. If the periodic profile is of a more general form, then three methods can be used: 1) By partitioning the grating into fine layers and approximating each one of these profiles by a rectangular profile. Now we have a multiple layered grating with the same periodicity, and the problem can be resolved as Section III. Although this extension is only an approximation for the original grating, it can be made as accurate as desired by subdividing the grating into sufficiently many fine layers. 2) By employing an exact numerical integration. 3) By averaging, for every value of $z$, the permittivity over $x$ inside the grating layer. Thus for a profile of the form

$$
x=h(z)=h(z+\Lambda), \quad \text { for } 0<x<T
$$

the averaged permittivity becomes

$$
\epsilon(z)=\epsilon_{2}+\left(\epsilon_{2}-\epsilon_{1}\right) h(z) / T .
$$

The problem is then reduced to that of a layer with uniform thickness, but with varying $\epsilon(z)$. This much simpler method is probably less accurate than the previous two.

In the case where the surface perturbation is small, simple approximation techniques and the coupled modes approach can be used. Marcuse [11], [125], determined the energy losses and the coupling in a dielectric guide with a slightly perturbed boundary by considering the boundary region as a thin inhomogeneous layer as we discussed above. A somewhat similar approach is to replace the boundary perturbation by an equivalent surface current [123]. If the field at the interface 


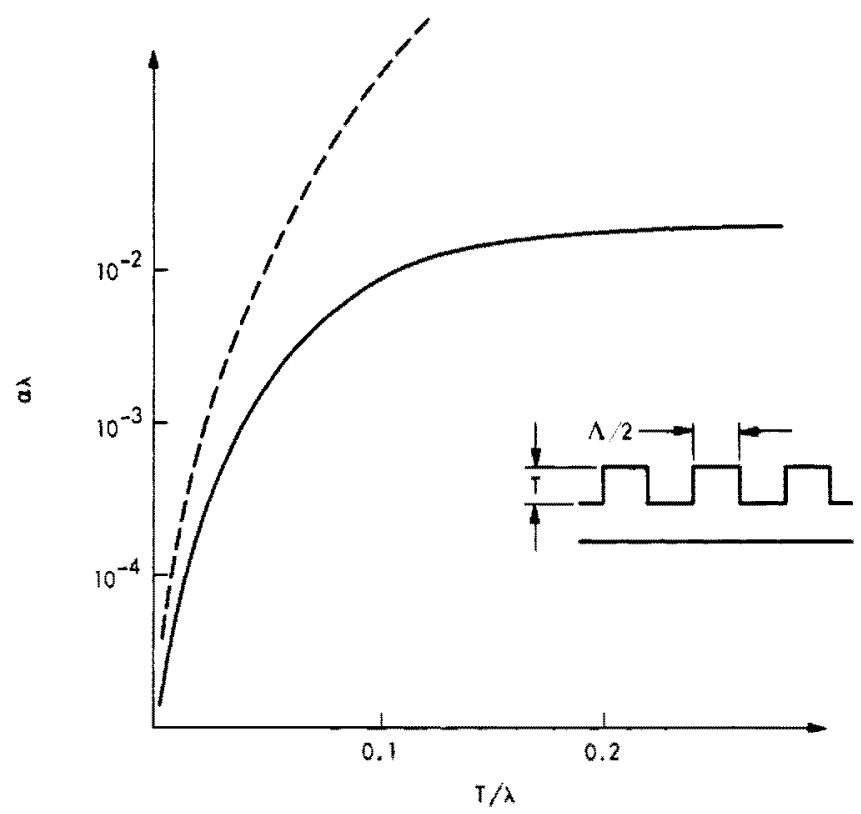

Fig. 17. Attenuation parameter in a dielectric guide with one boundary as a rectangular grating. The continuous line corresponds to the exact solution. The dashed line corresponds to an approximate solution using a perturbation method [119].

between two media of dielectric constant $\epsilon_{1}$ and $\epsilon_{2}$ is equal to

$$
E_{i}=C_{p} \exp i \kappa_{p}{ }^{2}
$$

then a small perturbation $T(z)$ of the boundary would have approximately the same effect as a surface current equal to

$$
\begin{aligned}
J_{s} & =-i \epsilon_{0} \epsilon_{1} \omega T(z) E_{i}+i \epsilon_{0} \epsilon_{2} \omega T(z) E_{i} \\
& =-i \epsilon_{0}\left(\epsilon_{1}-\epsilon_{2}\right) \omega T(z) C_{p} \exp i \kappa_{p} z .
\end{aligned}
$$

If $T(z)=T \cos (K z)$, then

$$
J_{s}=-i \epsilon_{0} \frac{\epsilon_{1}-\epsilon_{2}}{2} \omega T C_{p}\left[\exp i\left(\kappa_{p}+K\right) z+\exp i\left(\kappa_{p}-K\right) z\right]
$$

Consequently a reasoning similar to the one in the case of volume periodicity (Section III) would follow.

To check the accuracy of the approximate techniques discussed above, Peng et al. [119] computed rigorously the variation of the normalized attenuation parameter $\alpha$, which is due either to a stopband or to power leakage, for a square grating as a function of the grating thickness $T$ and compared it to a perturbation analysis by Ogawa $e t$ al. [142]. Peng et al. showed that the two methods give comparable results for $T<<\lambda$ where $\lambda$ is the wave wavelength. However, the perturbation method gives increasingly larger values, especially for $T \gtrsim 0.2 \lambda$ where the rigorous method shows that $\alpha$ reaches a saturation value (see Fig. 17). The behavior of the solid curve in Fig. 17 was explained by noting that the basic surface wave along a uniform layer $(T=0)$ has an evanescent field in the air region. When increasing $T$ from zero, the surface wave is perturbed by adding material on top of the thin-film waveguide. At first, this material appears in a region with strong fields, and therefore the effect on $\alpha$ is appreciable. However, as $T$ increases further, the additional material appears in regions where the field is gradually decaying, until at about $T / \lambda=0.2$ any further addition of material occurs in regions where the field is exponentially small. Consequently, the effect of increasing $T$ beyond $0.2 \lambda$ is negligible and $\alpha$ approaches a constant.
The difference between the rigorous approach and perturbation approach discussed above can be minimized if the perturbation is adequately weighted with the decaying exponential field away from the boundary.

The work on the effects of boundary perturbation was not limited to planar structures. Field distribution and mode conversion in circular dielectric and metallic guides with periodically changing diameters were analyzed in detail [143][145].

The scattering of acoustic waves from periodic gratings was also a subject of continued interest in the last 15 years. Waterman [146] studied the general problem of scattering of a scalar wave from a periodic surface. The problem of the diffraction of a plane-wave incident on thin periodically spaced parallel plates was solved many years ago by Carlson and Heiss [147], [148] using Wiener-Hopf methods, and by Berz [149] and Whitehead [150] using a residue calculus method from complex function theory. More recently, Desanto [151], [152] solved exactly the scattering of a plane wave by a onedimensional thin comb with soft (Dirichlet) and hard (Neumann) boundary conditions, where he used a residue calculus technique, and he illustrated the Rayleigh anomaly, Wood anomaly, and Brewster angle anomaly. He also [153] considered the case of periodically spaced thin parallel plates, where each period is divided by a further parallel plate into two regions which are, respectively, "free" and "filled." The filled regions have a wavenumber and density different from those of the surrounding media and the free regions. Both soft and hard boundary conditions were considered on the plates.

Another type of problem that has been considered is the propagation of acoustic waves in plane ducts with sinusoidally perturbed walls. Isakovitch [154] considered the case of a waveguide with one sinusoidal wall. Samuels [155] studied the case where both walls are sinusoidally perturbed in phase. Salant [156] generalized the previous work to the case where the phase difference between the perturbations on the two walls is arbitrary. They used a first-order expansion approach and found properties similar to the case of electromagnetic waves in perturbed waveguides. Nayfeh made a more detailed analysis of the resonance region [157] and added the effect of a mean flow in the duct [158].

An extension of the study of wave scattering from a periodically perturbed surface is the study of wave scattering from rough surfaces. A rough surface can be described by the spectrum of the roughness; thus it can be analyzed as a summation of a large number of periodic perturbations with specific weighting functions. The field of scattering from rough surfaces is out of the scope of this review. The reader is referred to a selected number of papers which cover the work in that area: Marcuse [111] for rough optical guides; Valenzuela et al. [159]; Tyler et al. [160] and Barrick [161] for scattering from the ocean surface; and Beckman [162] for scattering from rough surfaces in general.

\section{Source Radiation in Periodic Media}

Wave radiation from discrete electromagnetic sources in a periodic medium was considered by numerous authors. Casey [49] analyzed the radiation of electric and magnetic dipoles in a dielectric, and Elachi [51] extended the work to the case of plasma and uniaxial plasma. Singer and Tamir-Berman [50] studied the radiation from a filimentary source. Cerenkov and transition radiation were also investigated for the case of periodically stratified dielectric [46], [48], plasma [47], 
and uniaxial plasma [52]. The presence of the periodic perturbation leads to some special effects related to caustic surfaces and radiation cones. In this section, we review in some detail the formulation for the radiation of a magnetic dipole in sinusoidally periodic medium to illustrate the approach for resolving this type of problem. Then we briefly review other works in this field.

The solution for the radiation from a point or line source proceeds in a way similar to the cases of plane waves considered in Section II. The geometry studied by Casey [49] and Elachi [51] consists of an electric or magnetic dipole antenna located at $\rho=0$ and $z=z_{0}$ with the moment vector of the dipole directed along the $z$ axis. The entire space around it is filled with a lossless dielectric, where the permittivity $\epsilon$ is a periodic function of $z$. As a consequence of the symmetric geometry chosen, the electromagnetic field is independent of the azimuthal coordinate $\phi$.

In the case of a magnetic dipole, the radiated electric field satisfies the following wave equation outside the source

$$
\left(\frac{\partial^{2}}{\partial \rho^{2}}+\frac{1}{\rho} \frac{\partial}{\partial \rho}-\frac{1}{\rho^{2}}+\frac{\partial^{2}}{\partial z^{2}}\right) E-\mu_{0} \epsilon \frac{\partial^{2} E}{\partial t^{2}}=0
$$

where $\vec{E}=E \vec{e}_{\phi}$. Using the separation of variables method and the Floquet theorem, the solution can be written as

$$
E=\int_{-\infty}^{+\infty} W(\kappa) \sum_{n} E_{n} B_{1}(\delta \rho) \exp i(\kappa+n K) z d \kappa
$$

where the $E_{n}$ 's are given by the same recurrence relation as in (7) and (9), $B_{1}$ is

$$
B_{1}= \begin{cases}J_{1}, & \text { Bessel function for } \rho<a \\ H_{1}, & \text { Hankel function for } \rho>a\end{cases}
$$

and $a$ is the radius of the magnetic dipole loop. It is straightforward to see that the expression of $E$ is an integration over an infinite number of plane waves radiated in different direction with a weighting function $W(k)$. The dispersion relation:

$$
\Delta(\delta, \kappa, \omega)=\operatorname{det}\|M\|=0
$$

gives the value of $\delta$ as a function of $k$ for a fixed $\omega$. An example of the wave vector diagram (i.e., $\delta(\kappa)$ ) is given in Fig. 6 which shows that the solution is multivalued and, using the superposition principle, the field has to be written

$$
\begin{array}{r}
\vec{E}=\vec{e}_{\phi} \sum_{q=-\infty}^{q=+\infty} \sum_{n=-\infty}^{n=+\infty} \int_{-\infty}^{+\infty} W_{q}(\kappa) E_{n q}(\kappa) B_{1}\left(\delta_{q} \rho\right) \\
\cdot \exp i(\kappa+n K) z d \kappa
\end{array}
$$

where $\delta_{q}$ are the different values of $\delta$, and $E_{n q}$ corresponds to the amplitude of the $n$th space harmonic of the $q$ th mode. The relative values $C_{n q}=E_{n q} / E_{0 q}$ may be determined by solving (62). Taking $c_{q}(\kappa)=W_{q}(\kappa) E_{0 q}(\kappa) / \delta_{q}(k)$ the field expression becomes

$$
\begin{aligned}
& \vec{E}=\vec{e}_{\phi} \sum_{q=-\infty}^{q=+\infty} \sum_{n=-\infty}^{n=+\infty} \int_{-\infty}^{+\infty} c_{q} C_{n q} \delta_{q} B_{1}\left(\delta_{q} \rho\right) \\
& \cdot \exp [i(\kappa+n K) z] d k .
\end{aligned}
$$

The values of $c_{q}$ can then be derived from the source conditions [49], [51] which in this case is

$$
\left[H_{z}(\rho>a)-H_{z}(\rho<a)\right]_{\rho \rightarrow a}=I_{0} \Delta(z)
$$

where $I_{0}$ is the current in the loop, and $\Delta(z)$ is the delta function.

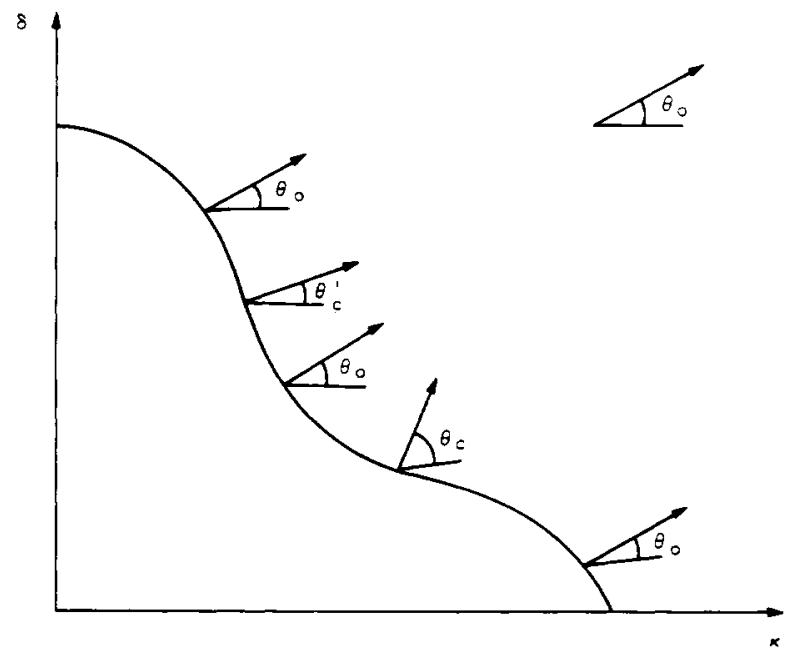

Fig. 18. A sketch of a wave vector diagram showing the saddle points (three in this case) which contribute to the far-field radiation in the direction $\theta_{0}$. It also shows two inflection points which would lead to caustics in the directions $\theta_{c}$ and $\theta_{c}^{\prime}$.

When $p \rightarrow \infty$, the electric field expression becomes

$E=-i\left[\frac{-2 i}{\pi r \sin \theta}\right]^{1 / 2} \sum_{q} \sum_{n} \int_{-\infty}^{+\infty} c_{q} \delta_{q}^{1 / 2} C_{n q} \exp \left[i r F_{n q}(\kappa)\right] d \kappa$

where

$$
\begin{aligned}
F_{n q}(K) & =(k+n K) \cos \theta+\delta_{q} \sin \theta \\
r^{2} & =\rho^{2}+z^{2} \\
\tan \theta & =\rho / z .
\end{aligned}
$$

Using the steepest descent method when $r \rightarrow \infty$, we find

$$
\begin{aligned}
E=-\frac{2 i}{r \sin \theta} \sum_{i} \sum_{q} \sum_{n} & \left(\frac{\delta_{q s}}{\delta_{q s}^{n}}\right)^{1 / 2} \alpha_{q s} C_{n q s} \\
& \cdot \exp \left[i r F_{q s}-i(\pi / 4)\left(1-\operatorname{sign} \delta_{q s}^{n}\right)\right] .
\end{aligned}
$$

Where the index $s$ means value at the saddle point, the summation over $j$ means summation over all the saddle points (it is possible to have more than one), and the prime corresponds to derivation relative to $k$. The saddle points are determined by

$$
\left.\frac{d F_{q}}{d \kappa}\right|_{\kappa=\kappa_{s}}=\left.0 \longrightarrow \frac{d \delta_{q}}{d \kappa}\right|_{\delta_{q}=\delta_{q s} \kappa=\kappa_{s}}=-\cot (\theta) .
$$

Thus, for a given radiation direction $\theta$, the field is mainly generated by the saddle points which are given by (68). This can be illustrated using the wave vector diagram shown in Fig. 18. For each value $\theta$, the saddle points can be graphically determined as the points where the normal to the wave vector diagram is in the direction $\theta$ relative to the horizontal axis. Of special interest is the presence of inflection points near the interaction regions between two modes (i.e., stopband). At these points, $\delta^{\prime \prime}=0$ and the field given by (67) is very large. This is a focalization and radiation enhancement effect due to the inhomogeneity of the medium. The corresponding conical surface of radiation is called "caustic." The caustic cone angle $\theta_{c}$ is given by

$$
\theta_{c}=\operatorname{arccot}\left(-\left.\frac{d \delta}{d \kappa}\right|_{\text {at infloction point }}\right) .
$$




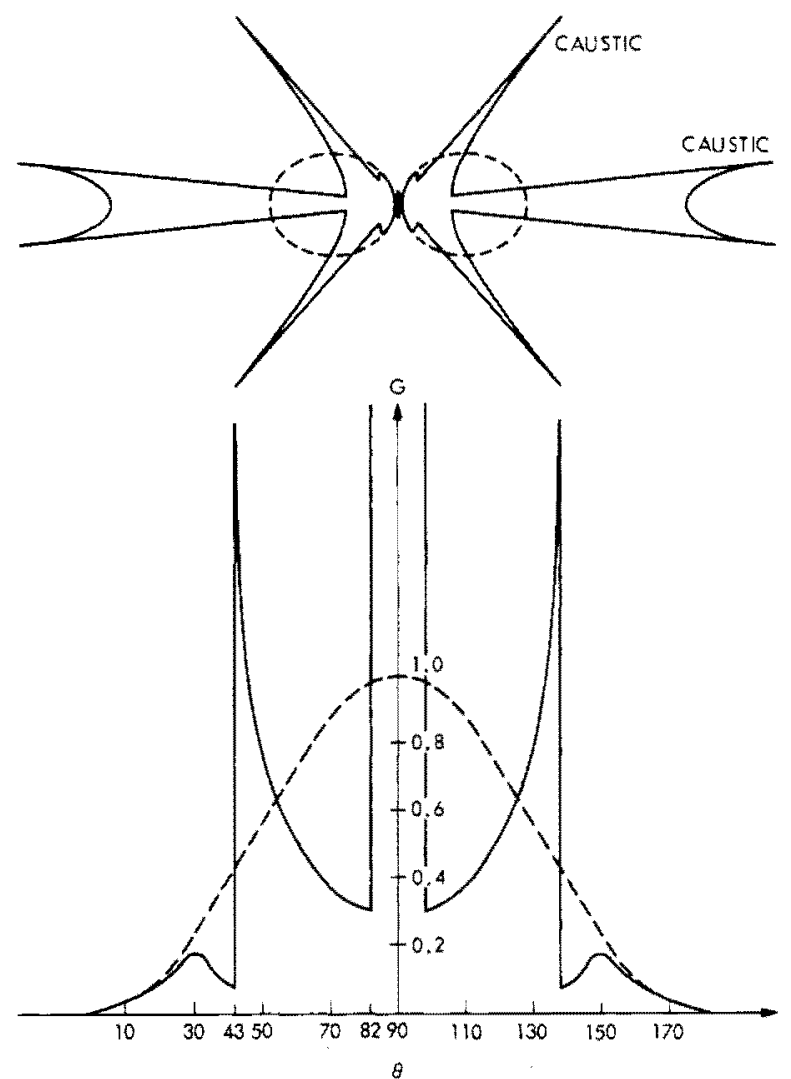

Fig. 19. Radiation pattern $G$ of a dipole in a sinusoidally modulated dielectric where the modulation is equal to $\eta=0.25$ and where $\lambda / \Lambda=0.8$. The dashed line corresponds to $\eta=0$.
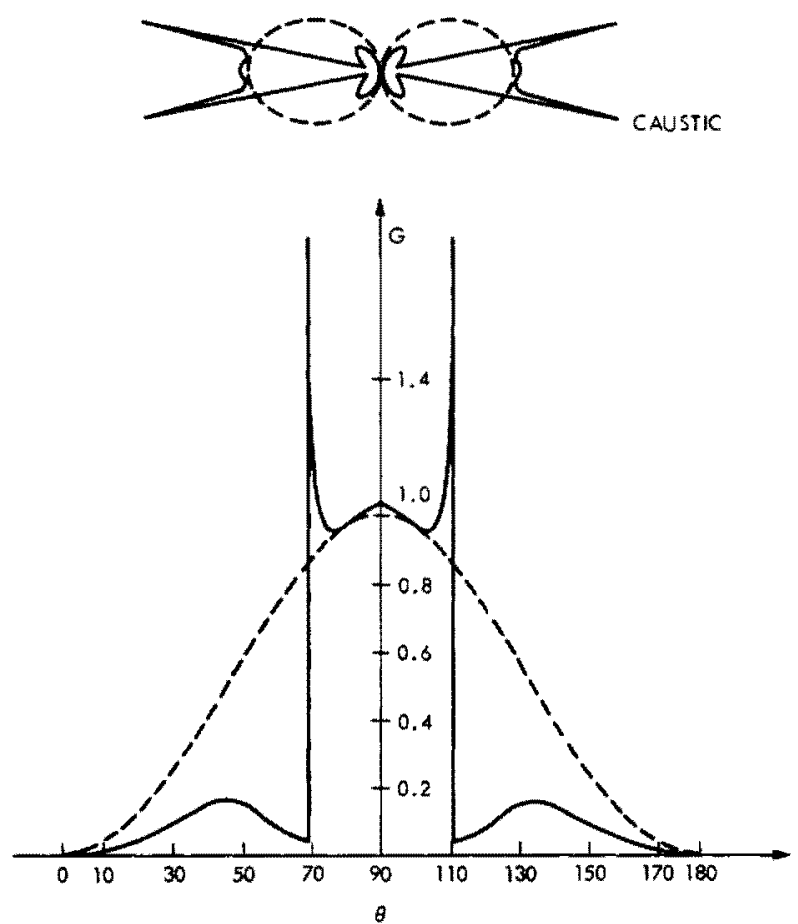

Fig. 20. Radiation pattern in a sinusoidally modulated uniaxial plasma where $\eta=0.25, \omega_{p o} / \omega=0.75$, and $\lambda / \Lambda=0.7$. The dashed line corresponds to the pattern in a vacuum.

In Fig. 19 we present an example of radiation pattern in a sinusoidally periodic medium where $\Lambda / \lambda=1.25$ and $\eta=0.25$.
There are four caustics at $\theta_{c}=43^{\circ}, 137^{\circ}, 82^{\circ}$, and $98^{\circ}$. For larger values of $\Lambda / \lambda$ more caustics appear.

The case of radiation in plasma and uniaxial plasma was also resolved. Of special interest is the result that in the case of a uniaxial plasma, radiation does occur outside the radiation cone (Fig. 20) under the same conditions where this is not possible in the case of a homogeneous uniaxial plasma [163].

A similar approach was used to study Cerenkov and transition radiation in a stratified dielectric, plasma or uniaxial plasma [46], [47], [49], [52]. In the case of the dielectric, owing to the inhomogeneity, the radiation given off by a charged particle moving uniformly through the medium contains not only Cerenkov-type radiation but also transitiontype radiation. The radiation is emitted at an infinite number of angles because of the presence of the space harmonics. Radiation is also present at all velocities of the particles because of transition-type radiation. Casey et al. [46] discussed also some interesting effects resulting from the Bragg reflections, such as Cerenkov radiation emission in the backward direction.

In the case of a periodically stratified plasma, only transition radiation is emitted. This case was analyzed in detail by Casey and Yeh [49]. They found that the emission is most pronounced in a band of frequencies beginning slightly above the average plasma frequency.

Another type of radiation from a moving particle in the presence of a periodic structure is the Smith-Purcell radiation. Smith and Purcell [164] demonstrated the generation of visible light by means of high-velocity electron beam grazing an optical grating. They ascribed the radiation mechanism to the formation of a vibrating dipole which consists of the moving charge and its accompanying oscillating image produced by the corrugated grating surface. Toraldo di Francia [165] offered an alternative explanation of this mechanism. He demonstrated that the radiation takes place via the various spectral orders of a grating, excited by the slow evanescent waves associated with a charge in its uniform rectilinear motion. Later, Hessel [166] used a formulation similar to the ones reviewed in this paper to derive a rigorous solution for the Smith-Purcell radiation from a reflection grating (represented by a plane with sinusoidally varying surface reactance) excited by a bunched, traveling current sheath. He specifically analyzed the presence of resonances which are similar to the Wood's resonances that we discussed previously. He also presented a graphical method for determining the location of the resonance using the Brillouin diagram.

\section{Transients in Periodic Structures}

Very little work has been done in the analysis of transients in periodic structures. Hill and Wait [167] studied analytically and numerically the scattering of an impulse and step excitation function by a grating of thin conducting cylinders. Elachi et al. [132] used the simple coupled modes approach to study the reflected and transmitted pulse when a rectangular or Gaussian pulse impinge on a periodic slab. The approach basically consists in getting the spectrum of the reflected and transmitted pulses using the expressions of the reflection $R(\omega)$ and the transmission $T(\omega)$ coefficients of the slab which were derived in (54). Thus the reflected pulse is

$$
r(t)=\frac{1}{2 \pi} \int_{-\infty}^{+\infty} G(\omega) R(\omega) \exp (-i \omega t) d \omega
$$



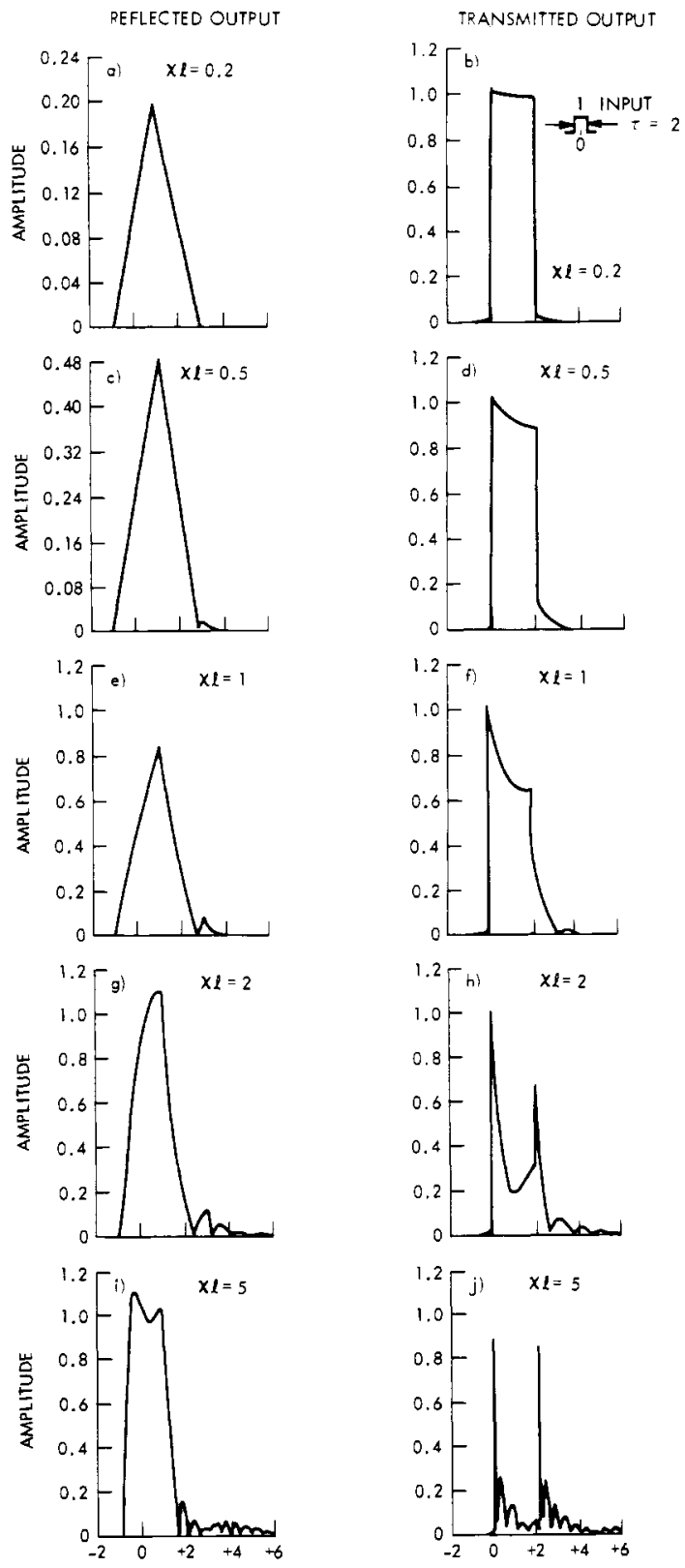

NORMAL IZED TIME

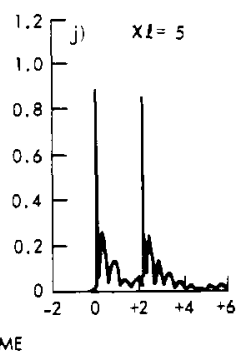

Fig. 21. Reflected and transmitted pulses for different values of coupling coefficient. Incident rectangular pulse has length of 2.0 time units and carrier frequency at Bragg condition. Note difference in vertical scales.

and the transmitted pulse is

$$
\rho(t)=\frac{1}{2 \pi} \int_{-\infty}^{+\infty} G(\omega) T(\omega) \exp (-i \omega t) d \omega
$$

where

$$
G(\omega)=\int_{-\infty}^{+\infty} g(t) \exp (i \omega t) d t
$$

and $g(t)$ is the incident pulse. The slab, which basically acts like a passband filter, may distort the pulse appreciably if the coupling coefficient is not excessively small (Fig. 21).

\section{Active Periodic Structures}

Probably, the major impetus in the renewed interest in periodic structures is the development of distributed feedback (DFB) lasers. In 1971, Kogelnik and Shank [54] reported the operation of a dye laser using a distributed feedback resonator which consists of a grating throughout the active medium. The periodicity was generated by exposing a thin film of dichromated gelatin to the interference pattern produced by two coherent ultraviolet beams from a helium-cadmium laser. The gelatin was then developed that resulted in a spatial modulation of the refractive index. The developed gelatin was then soaked in a solution of rhodamine $6 \mathrm{G}$ to make the dye penetrate into the porous gelatin layer. The resulting DFB structure was transversely pumped with the ultraviolet radiation from a nitrogen laser, and laser oscillation at $0.63 \mu \mathrm{m}$ was observed.

This led to a large number of experiments and papers on different types and varieties of DFB lasers (Fig. 22). Shank et al. [55] developed a tunable DFB laser. The feedback was obtained from a spatial modulation of both gain and index of refraction, induced by pumping a liquid organic dye solution (rhodamine 6G in ethanol) with fringes formed by the interference of two coherent beams from a ruby laser. The tunability was achieved either by varying the angle between interfering pump beams or the refractive index of the dye solvent. Hill and Watanabe [57] built a DFB laser where the active medium is an optically pumped organic dye solution film adjacent to a thin dielectric film with a periodically modulated index of refraction. This side-coupled DFB laser was operated in different modes: normal wave gain and feedback; normal wave gain and evanescent wave feedback; and evanescent wave gain and normal wave feedback. Fork et al. [58] used a nondestructively read-reversible optical memory material (photodimers of acridizinium ethylhexanesulfonate) to provide feedback in the form of an adjustable period phase grating which can be optically written (at $\lambda=0.364 \mu \mathrm{m}$ ) or erased (at $\lambda=0.313 \mu \mathrm{m}$ ). The lasing dye, the photodimers, and the supporting matrix are made into a single material by doping methylmethacrylate and acrylic acid with rhodamine $6 \mathrm{G}$ tosylate and photodimers of acridizinium ethylhexanesulfonate, and polymerizing the resulting solution to form a hard transparent plastic. The writing and erasing times were equal to $1 \mathrm{~s}$ and $4 \mathrm{~s}$, respectively. However, faster switching is physically achievable. Higher order Bragg gratings were also used as DFB resonator by Bjorkholm and Shank [59]. They showed that the resulting large decrease in the corresponding coupling coefficient (28) would require only a relatively small increase in the gain needed for oscillation. Higher order DFB cavities have a periodicity equal to a multiple of one-half the oscillation wavelength, and therefore are easier to construct.

The above types of DFB lasers used volume modulation of the complex index of refraction to achieve the distributed feedback. Another approach, which was proposed by a number of scientists [60] and first developed by Schinke et al. [61] consists in periodically varying the thickness of the waveguiding regions. The change in the guide thickness leads to a modulation of the longitudinal effective wave vector, thus it produces effects similar to refractive index changes in the guide medium. The surface grating was milled, with an argonion beam, into an $\mathrm{SiO}_{2}$ substrate through a Shipley $\mathrm{AZ} 1350$ photoresist mask which had been first exposed with an interference pattern produced by a single-frequency krypton laser 


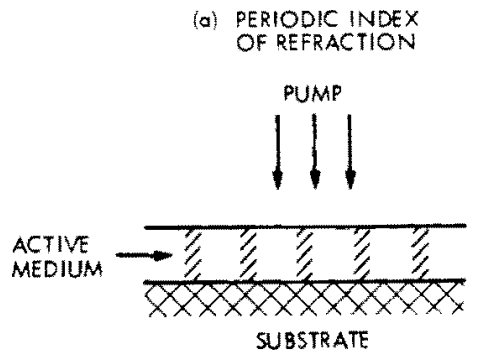

(b) PERIODIC PUMPING,
TUNABLE SCHEME

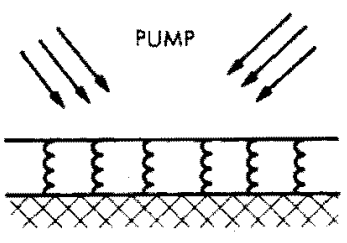

(e) ANISOTROPIC SUBSTRATE
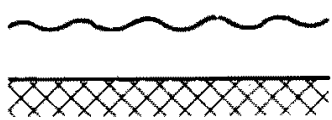

(9) SEMICONDUCTOR LASER, LIGHT PUMP

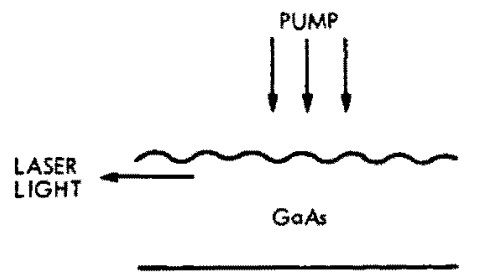

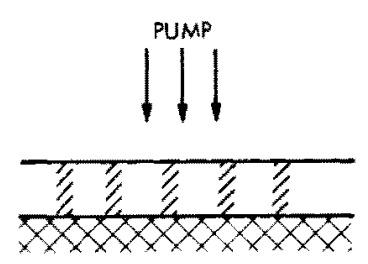

(h) SEMICONDUCTOR LASER. ELECTRIC PUMPING

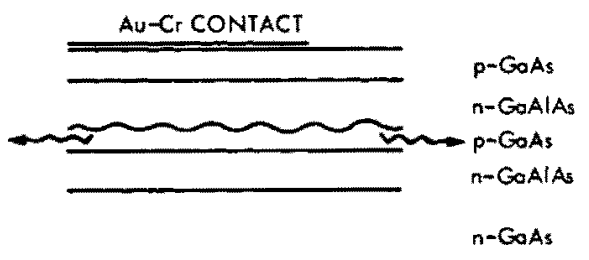

Au-Ge-NI CONTACT

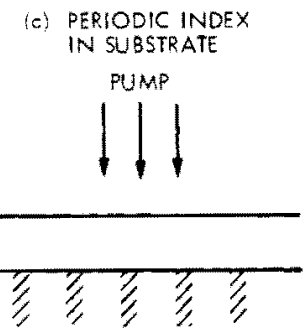

(f) ERASABLE DFE LASER

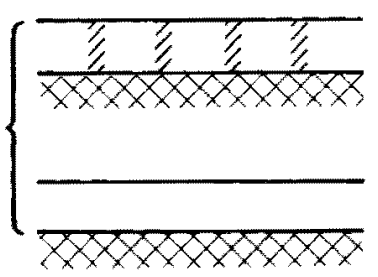

(i) RING LASER (i) ACTIVE MICROWAVE
CORRUGATION

(k) DBR LASER
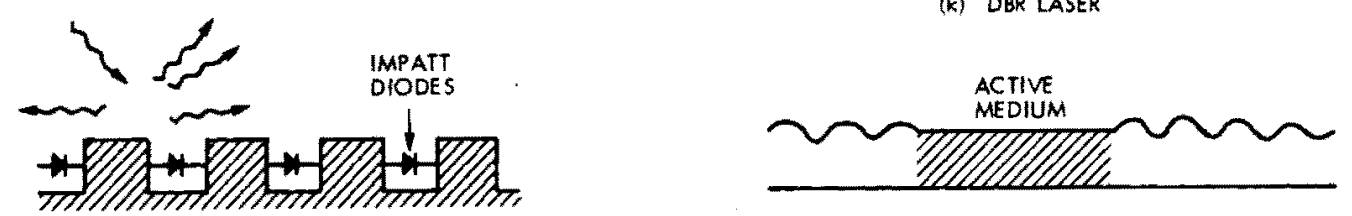

Fig. 22. Sketches of different active periodic structures which have been studied by a number of authors and are reviewed in this paper. Light-pumped DFB laser where (a) the active medium has a periodic index of refraction, (b) the pumping is done with the interference fringes of two beams from the same source, resulting in periodic gain, (c) the substrate has a periodic index of refraction, and (d) the upper boundary is periodically perturbed, (e) DFB Iaser in an anisotropic substrate, (f) erasable DFB laser. Semiconductor DFB lasers: (g) optically pumped and (h) electrically pumped, (i) ring DFB laser where the periodicity is over a part of the ring. (j) corrugated microwave structure with active mientr diodes in the corrugations (k) DBR (Distributed Brage Reflections) laser.

operating at $0.3507 \mu \mathrm{m}$, and then developed. The guiding medium was spin-coated polyurethane doped with rhodamine $6 \mathrm{G}$ to provide gain.

Bjorkholm et al. [64] and Kogelnik et al. [65] added another variety by depositing the DFB laser guide on an anisotropic substrate. This structure leads to hybrid Bragg scattering between oppositely traveling waves with orthogonal polariza- tion, i.e., mode conversion (see next section) and distributed feedback occur simultaneously. Wang and Sheem [66] extended the spatial periodicity to two dimensions, and developed a DFB laser with two-dimensional gratings with periods of 0.635 and $4.34 \mu \mathrm{m}$.

Optically pumped GaAs semiconductor surface DFB laser was first reported by Nakamura et al. [67]. The DFB laser 
was obtained by periodically corrugating the air GaAs interface with a corrugation period of $0.35 \mu \mathrm{m}$ and groove depth of $\sim 500 \AA$. Optical pumping was provided by a $Q$-switched ruby laser $(\lambda=0.6943 \mu \mathrm{m})$ and the DFB laser oscillated at $\lambda=0.83$ $\mu \mathrm{m}$. The same authors [69] then developed epitaxial GaAs waveguide DFB lasers which allowed controlled optical confinement. They also reported an optically pumped GaAs DFB laser with a corrugation of $0.11-\mu \mathrm{m}$ period [80]. Shank et al. [82] also developed a double heterostructure GaAs-GaAlAs DFB laser with $0.115-\mu \mathrm{m}$ periodic grating.

The logical second step was the development of electrically pumped DFB semiconductor lasers. The threshold analysis of a double heterojunction GaAs-GaAlAs DFB laser with an internal corrugation was conducted by Nakamura and Yariv [72]. Their results indicated the possibility of significant threshold reductions as well as of new possibilities of transverse mode control. A number of experimental developments of different configurations of electrically pumped semiconductors or DFB lasers were then reported. Stoll and Seib [85] demonstrated a distributed feedback GaAs hemojunction injection laser. The $p-n$ junction was formed by diffusing zinc into heavily n-type $\mathrm{GaAs}$, and the grating was ion machined into the surface of the p-type layer. The grating had a 0.35 $\mu \mathrm{m}$ periodicity for third-order Bragg feedback and $0.05-\mu \mathrm{m}$ groove depth. Nakamura et al. [84] developed a GaAs-GaAlAs double heterostructure diode DFB laser. They also used thirdorder coupling for laser oscillation. Anderson et al. [86] reported a double heterostructure GaAs injection DFB laser with a first-order coupling. Scifres et al. [93] used fourth-order coupling in a GaAs-GaAlAs single heterojunction diode.

The above lasers were operated at $77 \mathrm{~K}$. Reinhart $e t$ al. [94] reported the first room-temperature double heterostructure injection laser using distributed Bragg reflectors (DBR). Casey et al. [95] and Aiki et al. [96] used separate optical and carrier confinement to operate GaAs-GaAlAs heterostructure DFB lasers at room temperature.

Ring DFB lasers were also developed by Wang and Tsang [97]. Other types of DFB lasers were proposed by a number of authors. Marcuse [62] analyzed the use of DFB in capillary waveguide lasers. Elachi et al. [90], [124] studied DFB fiber lasers and DFB diffuse waveguide lasers. Fisher [87], Yariv [88], and Elachi et al. [89] proposed different schemes for the potential use of crystals as DFB cavities for X-ray lasers. The DFB concept was also studied by Elachi as applied to bulk and surface microwave acoustic oscillators [105], [106] and magnetic wave oscillators [104].

The theoretical analysis of the DFB concept was first given by Kogelink and Shank [56] using the coupled mode theory for the case of transversely unbounded, mirrorless structure. Their analysis was then extended by a number of authors. Chinn [71] and Streifer $e t$ al. [91] studied the effects of endmirror reflectivity. Elachi et al. [90] considered the case of transversely bounded DFB lasers. Wang [77] analyzed the distributed Bragg reflection (DBR) configuration where the corrugated structure and active medium are longitudinally separated. Wang et al. [73], [74], [77] used truncated Floquet harmonics and the method of multiple reflection to derive the threshold condition of DFB and DBR lasers. They discussed the relation between the truncated space harmonics and the forward backward waves used in the coupled modes theory. They pointed out that the labels "forward" and "backward" waves used in the coupled modes theory correspond to phase velocity and not direction of energy flow. Based on this, they claimed that the boundary conditions applied by other authors using the coupled modes approach are not correct. However, Yariv and Gover [92] did show that the two approaches are identical. In this section, we review the theoretical analysis of DFB lasers following the work of Kogelnik and Shank [56] and Elachi et al. [90] which used the coupled modes approach.

The coupled waves equations for a forward $p$ th mode wave $F_{p}(z) e^{i \beta_{p} z}$ coupled to a backward $q$ th mode wave $B_{q}(z)$. $e^{i \beta q z}$ in an active medium are similar to the ones for a passive medium. These equations are

$$
\begin{aligned}
& \frac{d F_{p}}{d z}-\left(C_{p} g+i \Delta \beta_{p}\right) F_{p}=i \chi_{p q} B_{q} \\
& \frac{d B_{q}}{d z}+\left(C_{q} g+i \Delta \beta_{q}\right) B_{q}=-i \chi_{p q} F_{p}
\end{aligned}
$$

where $g$ is the gain coefficient of the medium, $C_{p}$ and $C_{q}$ are efficiency factors which depend on the modes, $\chi_{p q}$ is the coupling coefficient that takes into account the transverse phase matching, and $\Delta \beta_{p}$ and $\Delta \beta_{q}$ are related to the frequency mismatch $\Delta \omega$ relative to the exact Bragg frequency $\omega_{p q}$ by

$$
\begin{aligned}
& \Delta \beta_{p}=\psi_{p} \Delta \omega=\left.\frac{\partial \beta_{p}}{\partial \omega}\right|_{\omega_{p q}} \Delta \omega \\
& \Delta \beta_{q}=\psi_{q} \Delta \omega=\left.\frac{\partial \beta_{q}}{\partial \omega}\right|_{\omega_{p q}} \Delta \omega .
\end{aligned}
$$

The solution for the above equation is given by (26). The boundary conditions which reflects the fact that the system must self-oscillate are

$$
F_{p}(-L / 2)=B_{q}(L / 2)=0
$$

in the absence of end reflections, where $L$ is the length of the DFB and active medium. Replacing $F_{p}$ and $B_{q}$ in (73) by their expressions from (26), and using the above boundary condition, we find

$$
\begin{aligned}
\Delta \kappa_{1}, \Delta \kappa_{2} & =\frac{C_{p}-C_{q}}{2} g+i \frac{\psi_{p}-\psi_{q}}{2} \Delta \omega \\
& \pm\left[\left(\frac{C_{p}+C_{q}}{2} g+i \frac{\psi_{p}+\psi_{q}}{2} \Delta \omega\right)^{2}\right. \\
& \left.+\chi_{p q}^{2}\right]^{1 / 2}
\end{aligned}
$$

and

$$
\frac{\Delta \kappa_{1}-\Delta \kappa_{2}}{2}= \pm i \chi_{p q} \sinh \left(\frac{\Delta \kappa_{1}-\Delta \kappa_{2}}{2} L\right) .
$$

The above equation is the threshold condition. Its solution gives the threshold gain $g L$ needed for oscillation and the frequency of oscillation $\omega_{p q}+\Delta \omega$. The solution is multivalued and gives many solutions for $\Delta \omega$ and $g$ which correspond to the oscillation spectrum. If the two interacting modes are identical, then $\Delta k_{1}=-\Delta \kappa_{2}$ and the threshold condition reduces to

$$
\Delta \kappa= \pm i \chi \sinh (\Delta \kappa L)
$$

where

$$
(\Delta K)^{2}=\left(C_{p} g+i \psi_{p} \Delta \omega\right)^{2}+\chi^{2}
$$


which is the equation originally derived by Kogelnik and Shank, and is valid for both index and gain coupling. This equation could have also been derived from the expression of the reflection coefficient $R$ [see Section III, (54)] of a periodic slab by taking $R \rightarrow \infty$ and replacing $i \Delta \beta$ by $C_{p} g+i \psi_{p} \Delta \omega$.

In the above derivation, we assumed that there is no Fresnel reflection at the two ends of the DFB structures. Chinn [71], Wang [77], and Scifres et al. [158] did analyze the effect of end reflectors in some detail. They showed that the presence of the reflectors does not necessarily decrease the threshold gain. The phase of the end reflection coefficient plays a major role, and it could even increase the threshold gain. This seems reasonable because the wave reflected at the ends has to add in the right way with the wave reflected by the grating.

Wang et al. [73]-[75], [77] used a different approach based on a truncated Floquet solution. They derived the oscillation condition for a variety of DFB configurations and DBR configurations. The DBR consists in having the periodic structure separated from the active medium, and positioned at the two ends, i.e., replace the mirrors in a conventional laser by gratings. They derived general formulas of which the different configurations are special cases.

The DFB and DBR cavities are especially useful in the field of thin-film and integrated optics. In those structures, it is extremely hard to build in end mirrors. It is far easier to construct a grating. These type of cavities have also potential for extremely high-frequency laser (i.e., X-ray region) where no mirrors are available, or for high-power lasers where mirror burning is a serious problem.

Most of the work in the field of active periodic structures was directed toward DFB and DBR oscillators. However, a few other applications were also considered. Yariv and Armstrong analyzed the use of periodic structures as traveling wave optical amplifiers and oscillators [169]. A number of authors analyzed the possibility of developing a solid-state traveling wave amplifier that would be similar to the vacuum TWT and would operate in the optical and submillimeter region. Although the idea sounds simple, there are problems of lowvelocity saturation of carriers. Solymar and Ash [170], Sumi [171], and Mayer and Van Duzer [172] considered the scheme where a current conducting semiconductor is placed in close proximity to an external slow-wave structure. Gover and Yariv [107] considered the case of a periodically corrugated interface in a monolithic way, which may be accomplished by diffusion, ion implantation, epitaxial growth, or carrier injection. This structure would allow tighter coupling between the carrier current and the slow electromagnetic wave. Experimental evidence for interaction between drifting carriers in n-type InSb and slow electromagnetic waves supported by meander-type and helix-type circuits at $77 \mathrm{~K}$ was reported by Sumi and Suzuki [173]. Superlattices (Section IX) were also suggested by Gover and Yariv [174] as structures which could be used to accomplish intraband radiative transition, and develop IR amplifiers and modulators.

Another field considered is the use of active corrugated structures, in which each slot region is connected to a microwave solid-state amplifier, as a reflecting antenna or a surface wave amplifier. Panicoli et al. [175] suggested a revolutionary spherical reflector antenna formed by a corrugated surface. By properly adjusting the depth of the corrugation on the reflector, and hence the phase of the reflection coefficient, it is possible to eliminate the coma aberration and to broaden its frequency bandwidth. Lee and Fong [176] suggested and analyzed an improvement to it by adding microwave solidstate reflection amplifiers, such as Gunn diodes or IMPATT diodes, in the slots. This would allow the control of both the amplitude and phase of the reflectivity. These degrees of freedom can be used to advantage in amplifying the incident wave, shaping the beam, correcting the aberrations and broadening the bandwidth. Lee and Fong also analyzed the use of the same structure as a surface wave amplifier. The wave is amplified as it propagates along the structure and interacts with the discrete active diodes. The main advantage of such an amplifier lies in the fact that, unlike the conventional amplifier operating in waveguides, the corrugated structure does not support higher order modes and, therefore, should minimize some stability problems for diode operations.

\section{Passive Periodic Structures}

Periodic structures also have considerable applications as passive structures. In the field of integrated optics and thinfilm optics they have been used as filters [177], [178], grating couplers [179]-[181], mode converters [182]-[185], modulators [186]-[188], beam deflectors [189]-[192], for acousticoptic interaction [193]-[196] for second harmonic generation [99]-[103], and frequency selective coupling [197]. They have been used extensively as transducers and filters in the field of integrated surface microwave acoustics [53], [198][210]. They were suggested for pulse compression [211], [212] and for generation and detection of thermal waves [213]. These structures are also commonly encountered in nature. Cholesteric liquid crystals (CLC) have a natural helical structure with a typical period of $1 \mu \mathrm{m}$. Biological filters are encountered in insects compound eyes. Natural and synthetic crystals are very common, and their interaction with electrons and waves has been studied intensively for many years. Other areas of investigation also include the flexural motion in periodic beams, periodically stiffened structures used in aeronautical and naval frame works, the propagation of acoustic waves in ducts with sinusoidally perturbed walls, and their scattering by thin plates. Some of these structures are sketched in Fig. 23.

\section{A. Filters}

The filtering property of periodic structures is basically a result of the presence of stopbands where the longitudinal wave vector becomes complex leading to exponential attenuation of the incident wave. The energy is reflected backwards over a finite frequency band. The efficiency of the filter is characterized by its relative bandwidth $\Delta \omega / \omega$ and coupling coefficient $\chi$. The transfer function of this filter is given by (54) and shown in Fig. 15. Recently Flanders et al. [177] have reported the development of a thin-film guided wave filter using surface corrugations. They achieved a reflectivity of 75 percent and a bandwidth of less than $2 \AA$ at $3 \mathrm{~dB}$. Multilayered optical filters have been known for many years [15], [16].

Acoustooptical interactions were also considered for optical filtering [178]. Of special interest is the tunable acoustooptic filter (TAOF), developed by Harris [216], [217], where a polarized optical wave parametrically interacts with a volume acoustic wave in an anisotropic crystal such as quartz or $\mathrm{CaMoO}_{4}$, leading to selective $90^{\circ}$ polarization shift for the optical waves which satisfy the Bragg frequency. An adequately positioned output polarizer would only transmit the rotated band of the input spectrum. This system is under 


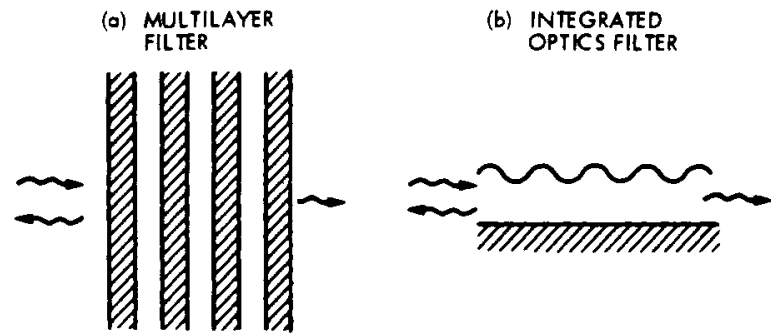

(e) INPUT COUPLER

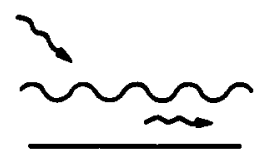

(i) LIGHT DEFLECTOR

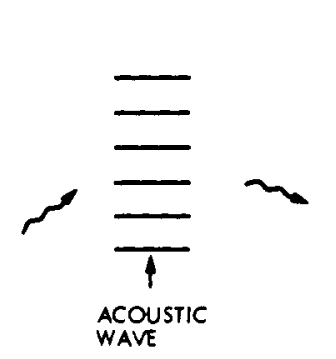
(I) 2-D COUPLED MECHANICAL PLATES

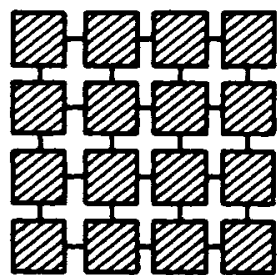

(f) OUTPUT COUPLER

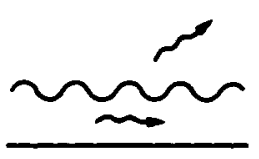

(i) SURFACE TRANSDUCER

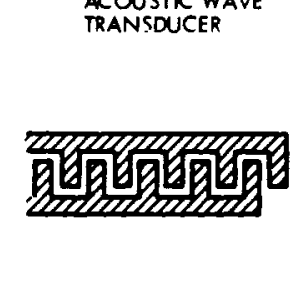
ACOUSTK WAVE (c) MODE MODE
CONVERTER

(d) ELECTRO-OPTK MODULATOR

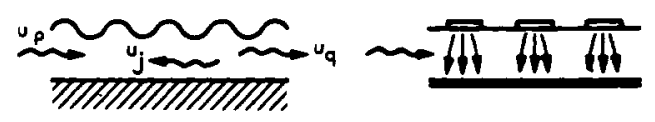

(g) SECOND HARMONIC GENERATOR
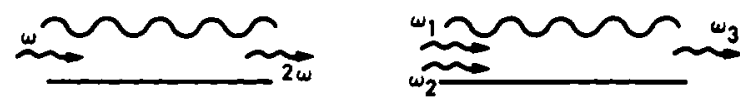

(h) FREQUENCY MLEER

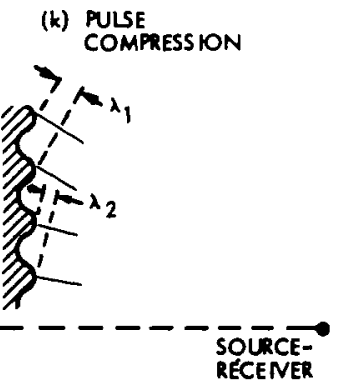

Fig. 23. Sketches of different passive periodic structures which have been studied in the literature, and are reviewed in this paper: (a) multilayer filter; (b) optical surface wave filter; (c) mode converter; (d) electrooptic modulator where the periodic perturbation is controlled electrically; (e) input coupler; (g) second harmonic generator; (h) nonlinear frequency mixer; (i) light deflector using acoustic waves to generate a controlled grating; (j) transducer for surface acoustic waves; (k) a scheme proposed for pulse compressor using a grating; (a) two-dimensional mechanical structure consisting of coupled plates; (m) multilayered cornea of the horsefly eye; (n) rhabdom of the eye of the Buckeye butterfly, consisting of periodic lisks in a guide; (o) section of the rhabdom of a skipper eye, consisting of a circular guide with corrugated surface.

consideration for a variety of applications including spacecraftborn spectrometers.

Most of the other applications are based on the fact that a periodic structure supports an infinite number of space harmonics with different longitudinal wave vectors.

\section{B. Mode Converters}

A transversally bounded structure supports a large number of guided modes of different longitudinal wave vectors $\beta_{i}$. Thus they are not cross-coupled. If the structure is periodic with a periodicity $\Lambda=2 \pi / K$ such that

$$
\beta_{p}-K=-\beta_{q}
$$

then the $p$ th mode is coupled, through one of its space harmonics, to the oppositely propagating $q$ th mode leading to energy transfer from the $p$ th to the $q$ th mode, i.e., mode conversion. The same effect could also occur between two codirectional modes if

$$
\beta_{q}+K=\beta_{q}
$$


with back and forth power transfer between the two modes. Mode conversions have been experimentally demonstrated in thin-film guides [182]-[185].

\section{Couplers}

The same principle is used in grating couplers. A plane wave incident on the surface of a thin-film grating excites an infinite number of space harmonics of wave vectors:

$$
\beta_{n}=k \sin \theta+n K
$$

where $\theta$ is the incidence angle. If one of these harmonics is phase matched (i.e., has approximately equal wave vector) with a guided mode, then efficient coupling occurs. Coupling efficiency as high as 40 percent has been demonstrated with gratings made from photoresist and fabricated directly on the film. The theoretical analysis is similar to the one discussed in Sections III and IV [179]-[181].

\section{Parametric Interactions}

Periodic structures were also suggested for parametric interaction and second-harmonic generation. Practically all nonlinear media have a frequency dependent index of refraction. This implies that colinear parametric interactions are not efficient because of phase mismatching, i.e.,

$$
\beta\left(\omega_{1}\right) \pm \beta\left(\omega_{2}\right) \neq \beta\left(\omega_{1} \pm \omega_{2}\right) .
$$

Thus parametric interactions usually require an anisotropic material or a bounded structure which supports many modes of different wave vectors [215]. However, the use of periodic structures allows controlled phase matching, because $K$ can be adjusted. For instance, in the case of second-harmonic generation, a structure with periodicity $\Lambda=2 \pi / K$ such that

$$
|2 \beta(\omega)-\beta(2 \omega)|=K
$$

would give phase matching and efficient second-harmonic generation. This concept was first suggested by Bloembergen and Sievers [99], and some varieties of it later studied by others [100]-[102]. This scheme allows the use of highly nonlinear but isotropic material for parametric interaction. Recently, Levine et al. [103] succeeded in accomplishing phase-matched second-harmonic generation at $\lambda=1.06 \mu \mathrm{m}$ (fundamental frequency) in a nitrobenzene filled waveguide using a periodic electrode, with period $\Lambda=13.6 \mu \mathrm{m}$, to modulate the nonlinear susceptibility.

\section{E. Deflectors and Modulators}

Periodic structures have also been developed and used for beam deflection and modulation. One could achieve periodic changes in the refractive index by the electrooptical effect using a modulation voltage applied to a periodic electrode pattern or by the acoustooptical effect using acoustic waves at appropriate frequencies. Since the changes of refractive index can now be controlled by either the applied voltage or the amplitude of the acoustic waves, this type of deflector or mode converter can also serve as a modulator or switch. Gia Russo and Harris [184] and Polky and Harris [187] demonstrated the electrooptical Bragg modulation at $\lambda=0.63 \mu \mathrm{m}$ in nitrobenzine waveguides. They were able to achieve up to 50-percent modulation. Switching and modulation of light using magnetooptical effects in garnet waveguide were accomplished by Tien et al. [188]. The magnetooptic effect was also used by Tsang et al. [185]. Experimental demonstration of acoustic deflection of optical surface waves at $\lambda=0.63 \mu \mathrm{m}$ was reported by Kuhn et al. [189] in glass waveguides at 191-320MHz acoustic frequency. Cheo and Reeder [192] demon- strated acoustic deflection at $\lambda=10.6 \mu \mathrm{m}$ in GaAs epitaxial waveguide. Many other authors reported acoustooptic deflection and scattering experiments. Acoustooptic interactions were also considered for optical mode conversion [182]-[184].

\section{F. Two-Dimensional Structures}

Another type of periodic structure that has been studied recently is the two-dimensional periodic metal mesh. Ulrich and Take [218] guided 337- $\mu \mathrm{m}$ waves in the form of TM surface waves along thin copper sheets perforated in a regular pattern. Wave propagation in two-dimensional surface periodic structures were also studied by Lee and Jones [219].

\section{G. Surface Acoustic Waves}

Periodic structures are also widely used in microwave acoustics as transducers, couplers, and filters. Interdigital transducers on piezoelectric surfaces are used for excitation and detection of acoustic surface waves [53], [198]-[202]. Gratings have been used for the coupling of volume and surface waves [203]-[205]. They are also used for filtering [206][209]. By combining a number of grating filters on a single substrate, Melngailis and Flynn [206] developed a 16-channel surface acoustic wave filter bank for real-time spectral analysis. Gratings with linearly varying periods were also developed to achieve a pulse compression filter with extremely high-time bandwidth product of 1500 [209]. For more detail about the use of periodic structures in surface acoustics the reader is referred to two general review papers [53], [201]. Blotekjaer et al. [210] recently gave a thorough theoretical analysis of acoustic waves in structures consisting of periodic metal strips on a dispersive medium using the Floquet formulation. They analyzed a number of different structures and the case of higher order Bragg scattering.

\section{H. Ultrasonic Light Diffraction}

The diffraction of visible light by ultrasonic compression waves propagating in a liquid was predicted by Brillouin in 1921 and observed a decade later by Debye and Sears, and Lucas and Biquard. Since then, many investigators have studied this phenomenon under a variety of experimental conditions obtained by varying one or more of the following quantities: incidence angle, wavelengths of the ultrasonic and light waves, amplitude of the ultrasonic wave; and width of the ultrasonic beam. An excellent review of the experimental and theoretical work in this area is given in the book Principles of Optics, by Born and Wolf [15], and in a paper by Klein and Cook [214].

\section{Ocean Waves}

Work was also reported on the propagation of ocean waves in periodic structures and their interaction with periodic boundaries. Rhines [220] studied the propagation of internal gravity waves in a periodic sheer flow, and the interaction of long gravity waves and Rossby waves with a corrugated bottom (i.e., boundary) and he discussed the stopband, passband effect on ocean waves.

\section{J. Pulse Compression}

In his book on the theory of sound, Lord Rayleigh [211] wrote: "At Tarling there is a flight of about 20 steps which returns an echo of a clap of the hands as a note resembling the chirp of a sparrow." This effect was simply explained by considering the backscattering of a noise signal (i.e., signal with a wide band) from an off-axis grating [Fig. 23(k)]. The portion of the signal with a larger value of $\lambda$ will be back- 
scattered by the grating cycles further away from the axis; thus they will take a longer time to reach back the receiver and the pulse is dispersed. Inversely, a chirped pulse is compressed. This scheme was suggested by Kock [212] to compress acoustic chirp pulses.

\section{$K$. Thermal Diffusion Measurement and Temperature Waves}

Eichlen et al. [213] considered the use of thermal phase grating to measure thermal diffusion and to excite and detect temperature waves (second sound) in solids. Two light waves with different directions of propagation were derived from a pulsed neodymium YAG laser and then superimposed in an absorbing sample to generate an interference field. Due to absorption, a spatially periodic temperature distribution occurs, producing a spatial modulation of the index of refraction which then can be considered as a thermal phase grating. The light of an argon laser simultaneously incident on the sample is diffracted by the thermal grating. When the excitation is eliminated, the decay time of the diffracted light is measured. From this decay time the thermal diffusivity of the sample is determined.

\section{Insect Compound Eyes}

Periodic structures do also appear in nature. Optical filters are present in the insect compound eyes. These eyes contain components that function as interference filters, diffraction gratings, multimode waveguides, and waveguide variable attenuators. The insect compound eye [221]-[226] is a closepacked collection of thousands of little eyes called ommatidia located at the surface of the insect's head. Each ommatidium possesses a set of optical components. The light is first incident on a lens (the cornea) of a $25 \mu \mathrm{m}$ diameter. It is then focused on the entrance of a transparent cylinder (crystalline tract) a few microns in diameter and hundreds of microns long. This tract has a refractive index greater than the index of its surrounding medium, thus it acts as a multimode waveguide. At the end of the guide is the rhabdom which plays the role of a photodetector. Many two-winged flies have their corneal lenses coated with a periodic set of layers [Fig. 23(m)] that cause brightly colored reflections when illuminated with white light [224]. These layers are of alternately high and low refractive indexes. They are about $0.1 \mu \mathrm{m}$ thick (i.e., $0.2-\mu \mathrm{m}$ period). Depending on the particular species, there are anywhere from six to over thirty layers which play the role of a rejection-band filter. Over the entire compound eye, a given species may have up to four different filter types, with different transmission characteristics as a function of wavelength, organized in various patterns of stripes or patches of a given filter type.

Other species, such as most butterflies, have a reflecting filter (the tapetum) which is at the bottom of each rhabdom. The butterfly tapetum is a periodic set of cytoplasmic plates that alternate with air space [Fig. 23(n)]. This is identical to a guide periodically loaded by dielectric discs. For white light propagating down the rhabdom waveguide onto the filters there is a large reflection for wavelengths within the filter rejection band. The reflected wave propagates up the rhabdom and out of the eye. where it can be observed as colored eyeshine. Light of other wavelengths simply propagates through the filter and is absorbed in the basal pigment. A typical value for the cytoplasmic plates period is about $0.25 \mu \mathrm{m}$ in the Buckeye butterfly eye. However, neighboring rhabdoms could have entirely different reflection properties.

A third type of optical filter which is commonly found in the skipper's eyes consists of rings around the rhabdom wave- guide [Fig. 23(o)] with axial periodicity of about $0.18 \mu \mathrm{m}$. This is identical to an optical fiber with a periodically corrugated surface.

The corneal layers, the butterfly tapetum, and the skipper rhabdom all change the spectral response of the photodetector. Their purpose seems to enhance the contrast of colored objects in a background of dissimilar color.

\section{Cholesteric Liquid Crystals}

Hundreds of papers and textbooks have been written about waves and particles in periodic, almost periodic, and disturbed crystals. So we will not review this field except briefly in the next section. However, here we will briefly review the work on cholesteric liquid crystal because of their unique properties.

Cholesteric liquid crystals (CLC) can be represented by a structure consisting of molecules arranged in thin anisotropic layers with the successive layers rotated through a small angle, leading to a spiral configuration [Fig. 1(q)] [227], [228]. These crystals have a very strong rotary factor: $60000 \mathrm{deg} / \mathrm{mm}$ compared to $300 \mathrm{deg} / \mathrm{mm}$ for ordinary organic liquids. They are characterized by a dielectric tensor:

$$
\left\|\begin{array}{ccc}
\bar{\epsilon}+\eta \cos K z & \eta \sin K z & 0 \\
\eta \sin K z & \bar{\epsilon}-\eta \cos K z & 0 \\
0 & 0 & \epsilon_{3}
\end{array}\right\|
$$

where $K=4 \pi / \Lambda$ and $\Lambda$ is the pitch of the spiral. This dielectric tensor can be represented by an ellipsoid of principal axes $\epsilon_{3}$ (parallel to $z$ ), $\bar{\epsilon}+\eta$ and $\bar{\epsilon}-\eta$. The ellipsoid spirals around the $z$ axis with a pitch $\Lambda . \epsilon_{3}, \bar{\epsilon}$ and $\eta$ are constants which characterize the crystal. The value of $\Lambda$ is usually of the order of $1 \mu \mathrm{m}$.

The analysis of optical wave propagation in such a structure proceeds in exactly the same way as discussed for the Floquet approach in Section II, except that we use the tensor expression of $\epsilon(z)$. The analysis done by Elachi and Yeh [229] did show that these structures have a unique stopband behavior. Depending on the relative values of $\epsilon_{3}, \bar{\epsilon}$, and $\eta$ there could be one, two, or three first-order Bragg stopbands when the incidence angle is changed from $0^{\circ}$ to $90^{\circ}$. This can be contrasted to the fact that only one first-order Bragg stopband exists in all the cases studied in this paper. The presence of multiple first-order stopbands is a result of the anisotropy $\left(\bar{\epsilon} \neq \epsilon_{3}\right)$, which implies that the medium can support two different eigenmodes. The three stopbands are a result of the inter- and cross-coupling of these eigenmodes.

Cholesteric liquid crystal have been used for a variety of optical filtering functions [230], [231]. These include notch, multinotch, bandpass, and multibandpass capabilities, extending over a wide wavelength range from the near ultraviolet to the far infrared. Furthermore, the fact that the pitch of the spiral configuration is a function of temperature, pressure, added chemicals, etc., may permit numerous other applications. Many experimental and theoretical papers on the subject have been published [227]-[239]. These crystals have also been used for phase-matched third-harmonic generation [239].

\section{N. Mechanical Structures}

Work on periodic structures was also undertaken in the fields of structural, mechanical, and acoustical engineering. Cramer and Leilich [240] studied flexural motion in periodic beam structures, and showed that waves can propagate in some frequency bands but not others. Heckl [241] considered a system of beams coupled together to form a regular grillege and 
demonstrated the same property. He studied the propagation of bending, longitudinal, and torsional waves. Mead [242] included the effects of damping in the wave propagation theory for periodic beams, and later [243] discussed the nature of the propagating waves and their possible interaction with acoustic waves. He specifically considered the case of a beam on regularly spaced rigid supports and transversely elastic supports. He also considered the coupling between converted pressure fields, such as those associated with farfield jet noise or turbulent boundary layer pressure fluctuations, and the periodically supported beam. Of special interest was the case where pressure field velocity is equal to the phase velocity of one of the eigenwaves on the beam leading to strong coupling. This coupling condition, which is well known in physics and electrical engineering, is called the "coincidence" excitation condition by mechanical and structural engineers. He demonstrated that at relatively low convection velocity, the pressure field could simultaneously excite two positive and one negative group velocity waves of different frequencies, and he explained a scheme where a low-speed convected flow can excite, indirectly, a wave in the beam having sonic wave velocity, which could then radiate acoustic waves. This work has then been followed [244] by a study of the harmonic and random responses of periodic beams on elastic supports and subjected to convected loading. The objective of his work was to analyse the damaging effect of vibration which could exist in the use of aerospace structures stiffened at regular intervals by identical stringers and excited by an intense noise field, or in the use of large reinforced plates in heat exchanges systems of nuclear power stations. Sen Gupta [118] also showed how natural frequencies of finite beam-type periodic structures can be found from the wave propagation characteristics.

The theoretical analysis of the vibration of beam-type structures was based on the difference equations approach which is adaptable to the discrete nature of these structures [117]. The approach basically consists in determining the transfer matrix of a unit element which is then repeated to simulate the whole system. This is similar to the approach used to study the transmission properties of optical multilayer filters [15]. The resulting general properties are similar to the properties of the other periodic structures reviewed in this paper.

\section{Waves and Particles in Crystals}

Many phenomena concerned with waves and particles in crystals follow a very similar theoretical pattern as we discussed before for the case of electromagnetic waves in periodic media. As a matter of fact, most of the impetus in the study of periodic structure in the first half of this century was in the fields of Bragg scattering in crystallography and energy bands in solid-state physics. Slater [245] wrote a review paper on the work in the field of interaction of waves in crystals as of 1958. The reader interested in that aspect of the field of waves in periodic structures is referred to that review paper or to a wide range of text books [246]. In this section we will only review the most recent and unique work on some types of crystals which seem to be very promising for new future applications and theoretical development. We will briefly discuss the work on superlattices, zeolite crystals, and disordered systems.

\section{A. Superlattices}

A new type of periodic structures which have been recently developed and are attracting considerable attention are the superlattices. They consist of accurately deposited thin layers (from $10 \AA$ up to few hundred Angstroms) of two different semiconductors of matching lattice constants. When these man-made electron potential square wells are built up into stacks of 10 to 100 periods, they essentially constitute an infinite configuration (because of the finite mean free path of the carriers) known as a superlattice. These structures open the possibility of creating quantum states with predetermined energy levels and bandwidths. The superlattices built to date consist of GaAs layers which form the "well," and GaAlAs layers which form the "barrier" of the square well. These two semiconductors are extremely closely matched in lattice constant. These structures are built by a molecular beam epitaxy technique. Their properties have been recently explored by a number of scientists. Esaki and Chang [247] studied their current transport properties which show nonlinear characteristics and exhibit an oscillatory behavior beyond a certain threshold voltage. Tsu et al. [248] measured the photocurrent in a superlattice and observed peaks of photon energies which correspond to transitions between quantum states in the valence and conduction bands. As a function of the applied voltage, the photo current exhibits pronounced negative differential conductance when the potential energy difference between two adjacent wells of the superlattice exceeds the bandwidth of the quantum states. Van der Ziel et al. [249] reported laser oscillation from optically pumped multilayer heterostructures of the above type. Dingle et al. [250] demonstrated the evolution of resonantly split discrete well state into the lowest band of a one-dimensional superlattice. They monitored the evolution of GaAs absorption spectrum as the number of coupled wells has increased from one to ten or more. Structures with ten or more coupled wells appear to approximate the superlattice regime, whereas structures with fewer wells are well described in terms of interacting single wells. They interpreted their experimental data with an exact solution of the Schroedinger equation for transmission through multiple rectangular potential barriers.

It should also be mentioned that recently Gover and Yariv [174] discussed the possibility of using semiconductor superlattices for intraband radiative transitions and for the development of infrared amplifier and modulators.

\section{B. Zeolites}

Another type of crystals which have unique properties and which attracted some interest recently are the zeolite crystals [251], [252]. They are naturally occurring or synthetic aluminosilicate porous structures, commonly termed molecular sieves, with one-, two-, or three-dimensional channels having minimum pore diameters of 3 to $12 \AA$. These channels are formed by the juncture of large sodalite cage units that are arranged in a periodicity analogous to that of simple atomic and molecular crystals. This leads to a periodic change in the diameter of the channels with periodicities from a few angstroms to few tens of angstroms. These structures have been recently proposed as potential DFB cavities for X-ray lasers [89].

\section{Disordered Crystals}

Recently a number of papers appeared in the solid-state physics field which extend the field of periodic structure to the study of multiperiodic structures, almost periodic structures, and disordered structures. Sah and Srivastava [12] considered a generalized diatomic lattice whose unit cell con- 
sisted of any combination of $A$ atoms and $B$ atoms. This was a generalization of the work by Kerner [253] who considered an array of purity atoms with impurity atoms periodically interspaced. Most of the work in the study of binary alloys was directed toward the analysis of the Saxon-Hutner theorem and its converse. The theorem basically states that a level (i.e., frequency) which is forbidden (i.e., in a stopband) in the infinite one-dimensional lattice formed of pure type- $A$ potentials and in that formed of pure type- $B$ potentials, is also forbidden in any arbitrary substitutional alloy. of $A$ and $B$. The original conjecture by Saxon and Hutner refers only to $\delta$-potentials of the Kronig-Penney type situated at the centers of equal cells, and it has been proved by Dworin [254]. Kerner [253] showed that the above theorem does not hold for square-well potentials. Landaur and Hellund [255] and Hori [256] demonstrated that the theorem may not be valid for potentials other than $\delta$ potentials. More recently Tong [11] showed that the Saxon-Hutner theorem and its converse are not necessarily true for all kinds of potentials, and he gave the condition required for the theorem to be valid. Other workers analyzed the case of disordered alloys [257] and almost periodic structures [258].

\section{Fabrication Techniques}

A review of the different techniques for fabrication of periodic structures is somewhat out of the scope of this paper. However, in this section, we will briefly review the techniques which have been developed in the last few years to construct extremely small period surface and near surface gratings for surface acoustic wave and thin film optical devices. A recent review paper was given by Smith [259]. The reader who is interested in the details is referred to that excellent paper.

The basic principle of the fabrication technique of surface gratings are illustrated in Fig. 24. A substrate surface is coated with a radiation-sensitive polymer film and exposed to radiation of spatially periodic intensity with the desired period. Following exposure, a development step removes either the exposed or unexposed polymer, thereby leaving the periodic pattern in relief on the substrate surface. The substrate itself can then be patterned either by etching a relief structure in it, by chemically doping the patterned areas, or by depositing a material into the grooves of the polymer relief pattern. A number of simple variations and additions can be made to the above process.

The formation of the pattern in the polymer film is usually accomplished by photolithography, electron lithography or $\mathrm{X}$-ray lithography. In photolithography a photo-resist which responds to visible or ultraviolet light is used. The periodic pattern may be formed by a holographic technique where two laser beams (from the same source) are interfered together to form interference fringes. This technique of producing gratings was first proposed by Rudolph and Schmohl [260] and it provides accurate, precise, and low-distortion gratings. Recently Shank and Schmidt [168] have reported gratings with periodicity as fine as $1108 \AA$. Another technique is the use of a pattern which can be imaged onto the coated substrate (projection printing) usually with some demagnification, or it can be used in contact with the surface (shadow printing). These techniques can be used to generate gratings of a periodicity of few microns. For finer gratings, the diffraction effects lead to distortions. This is eliminated by the use of $X$-ray lithography where shorter wavelength radiation is used $(10 \AA)$. Recently, gratings of $0.36-\mu \mathrm{m}$ period produced by holographic

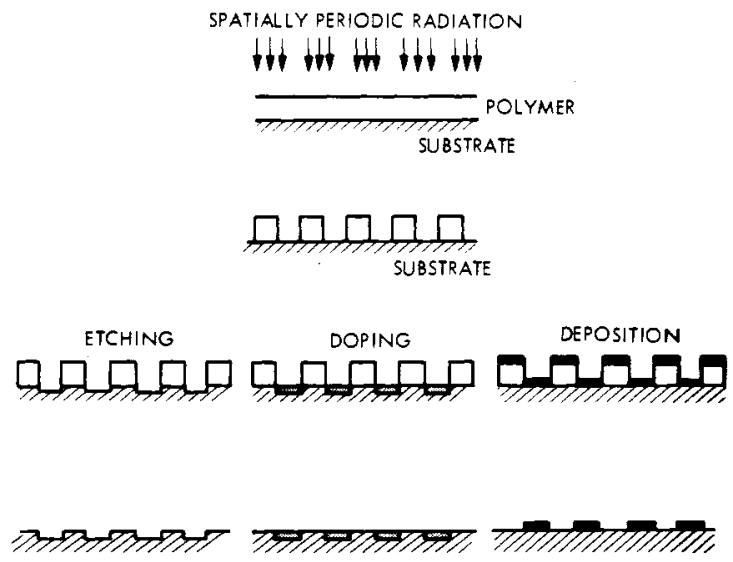

Fig. 24. Different techniques and steps to fabricate a surface grating [259].

means on an X-ray mask, were replicated in PMMA (polymethylmethacrylate) [261]. The third technique, electron lithography, uses an electron beam transmitted through a mask or a beam which is programmably scanned. Gratings of periods as small as $0.3 \mu \mathrm{m}$ are readily fabricated in PMMA films. Single lines of $0.045 \mu \mathrm{m}$ width have been written. However, no one has yet made 1-to-1 gratings with such line widths, primarily because of problems caused by electron scattering.

The etching or removal of material from the substrate can be achieved by ion bombardment (removed by sputtering) [262]. Such etchings exhibit extremely high-resolution capability $(0.1-\mu \mathrm{m}$ gratings [168]). Chemical etching can also be used, however it exhibits undercutting and is generally of low resolution. A detailed review of the ion implantation techniques was given by Gibbons [263], [264].

\section{Future Advances and Problems}

The study of waves in periodic structures has been active since the end of the last century, and it was applied to an amazing variety of fields, as we discussed in this paper. It seems that every few years a new advance in a field of science refuels the interest in the study of periodic structures, the most recent being the fields of integrated optics, DFB lasers, and superlattices. In this section, we speculate about the areas where we think the interest in theoretical analysis and experimental development will be highest in the immediate future. Some areas are already under investigation. Some that have not yet been investigated seem to be promising. We would like to emphasize that some of the following discussions are purely speculation, and the reader should consider them as such.

The field of DFB lasers have been active for the last few years and is expected to be so in the near future. There is need to undertake a Floquet analysis of active periodic structures especially as related to DFB lasers with surface periodicity, because deep groove gratings can be achieved with presentday technology. Similarly, the effects of leaky wave radiation on DFB lasers have not been analyzed. An extensive analysis of active periodic guides similar to what was done for periodic antennas might be rewarding.

Of potential interest is the phase-matched coupling between a guided wave and a leaky wave parallel to the surface. As we mentioned in Section III and sketched in Fig. 13, near that phase-matching point there are two real solutions. One corre- 
sponding to the guided wave, the other to the leaky wave which, in that narrow region, is completely "bound" as a result of the coupling. The question is whether or not these two waves can be used as a distributed feedback pair leading to DFB laser oscillation at some of the Wood or Raleigh frequencies.

Another area of interest is the potential use of crystal structures as DFB cavities for X-ray lasers. This scheme has been suggested, but theoretical and experimental work is still needed to evaluate its use.

The DFB concept has been suggested for the development of surface acoustic wave and magnetic wave oscillators. The same idea might also be applicable in other fields-for instance, the use of the active corrugated antenna analyzed by Lee and Fong [176] as a DFB oscillator in the microwave region. The structure basically consists of a corrugated metallic structure with amplifying diodes in the grooves.

The DFB concept might be used in self-sustaining lasers. It is well known that in conventional high-power lasers, the stationary wave in the active medium leads to modulation of the gain and probably the index of the medium. The question is, could this modulation itself play the role of a grating which would lead to distributed feedback of the light that generates it? In other terms, could the light wave generate its own feedback? This idea opens wide the whole area of the study of nonlinear DFB lasers and nonlinear coupling.

Most of the work in the field of periodic structures was directed toward multidimensional structures with one periodicity. Some work has been done in solid-state physics in the analysis of double or multiperiodic potentials which resulted in a number of controversies. This field seems to be of some interest both for theoretical and experimental investigations. A multiperiodic grating can be used for multifrequency DFB lasers or filters. Some basic questions are still not well understood concerning the stopbands of these structures, especially the ones resulting from multiple scattering. That is, for a medium where

$$
\epsilon(z)=\epsilon\left[1+\eta_{1} \cos K_{1} z+\eta_{2} \cos K_{2} z+\cdots+\eta_{n} \cos K_{n} z\right]
$$

a wave can be scattered successively by more than one of the above periodicities. This effect is intensively used in radar oceanography (over the horizon radar) in the remote study of ocean wave spectra. A number of interesting cases may be envisioned; for instance, the case of almost periodic functions.

The field of superlattices is another exciting one. The fact that it allows the development of lattices with specified band gaps is very interesting. This and other areas mentioned above opens a new and challenging field for the theoreticians. Could we determine a uni- or multiperiodic structure which would give a specified set of stopbands and passbands? Evidently, as most inverse problems, the solution, if any, is not simple. This field would have a wide range of applications in superlattices, integrated optics, integrated acoustics, and structural engineering.

Another area of interest is the analysis of transients and beams in periodic structures. Very little work has been undertaken in this field; a variety of problems in antenna theory, integrated optics, and integrated acoustics can be envisioned.

We would like to mention the extension of the work in periodic structures to cylindrical and spherical geometries. Even though this field seems of academic interest for the time being, unexpected applications might spring up in the future.
One which comes to the mind of the author is a recent paper by Schloessin [265] who suggested that the spherical boundary between the Earth's mantle and core is periodically perturbed by convection cells. This, if true, might have implications on the propagation of seismic waves in the Earth's body.

A possible application in planetary science is the fact that periodic plasmas have a passband at frequencies below the plasma frequency. It is well known that the Earth's ionosphere acts as a stop screen for all electromagnetic radiation of frequency below about $10 \mathrm{MHz}$ coming in from outside our planet. However, presently available high-power radars [266] could perturb the ionosphere in a periodic fashion. Thus it might be possible to punch a "hole" in the ionosphere allowing the passage of some radiation for radio-astronomical studies.

\section{CONCLUSION}

The field of waves in periodic structures has been active since the late part of the last century. In this review paper we covered the theoretical techniques which are used in studying the wave propagation and source radiation properties in unbounded and bounded periodic media. These techniques can be applied to all types of waves; electromagnetic, acoustic, magnetic, electron, ocean, internal, temperature, flexural, and mechanical waves. The passband, stopband, and space harmonic properties are common to all of them. The variety of applications reviewed in this paper do show the commonality between these fields. This common approach allows the transfer of concepts developed in one field to another, and may result in fruitful new advances.

As speculated in Section XI, the field of waves in periodic structures will be very active in the near future. New technological developments will continue a strong interest in this field, as has happened often times in the past.

\section{APPENDIX A}

\section{Wave Equations in a Periodic Medium}

\section{Electromagnetic Waves}

The source free wave equations in a medium whose permittivity is a function of the axial coordinates are [35]

$$
\begin{array}{r}
\nabla \times \nabla \times \vec{E}-k_{0}^{2}\left[\epsilon(z) / \epsilon_{0}\right] \vec{E}=0 \\
\nabla \times \nabla \times \vec{H}-[\nabla \epsilon(z) / \epsilon(z)] \times \nabla \times \vec{H}-k_{0}^{2}\left[\epsilon(z) / \epsilon_{0}\right] \vec{H}=0
\end{array}
$$

where $k_{0}^{2}=\mu_{0} \epsilon_{0} \omega^{2}, \epsilon_{0}$ and $\mu_{0}$ are the free-space permittivity and permeability, $\vec{E}$ and $\vec{H}$ are the electric and magnetic field vectors and $\epsilon(z)$ is the permittivity of the inhomogeneous medium. It can be shown that all field components in this medium can be obtained from the scalar quantities $\Phi_{1}$ and $\Phi_{2}$ as follows [35]:

$$
\begin{aligned}
& \vec{E}=\nabla \times\left(\Phi_{1} \vec{e}_{z}\right) \\
& \vec{H}=-\frac{i}{\mu_{0} \omega} \nabla \times \nabla \times\left(\Phi_{1} \vec{e}_{z}\right)
\end{aligned}
$$

for transverse electric waves; and

$$
\begin{aligned}
& \vec{E}=\frac{i}{\omega \epsilon(z)} \nabla \times \nabla \times\left(\Phi_{2} \vec{e}_{z}\right) \\
& \vec{H}=\nabla \times\left(\Phi_{2} \vec{e}_{z}\right)
\end{aligned}
$$


for TM waves. $\vec{e}_{z}$ is the unit vector in the $z$ direction. From the above equations, and separating the variables in rectangular coordinates, one obtains

$$
\Phi_{1}=\left\{\begin{array}{l}
\sin \\
\cos (s x)
\end{array}\right\}\left\{\begin{array}{l}
\sin (w y) \\
\cos
\end{array}\right\} U(z)
$$

and

$$
\Phi_{2}=\left\{\begin{array}{l}
\sin \\
\cos
\end{array}(p x)\right\}\left\{\begin{array}{l}
\sin \\
\cos
\end{array}(q y)\right\} V(z)
$$

where $s, w, p$, and $q$ are separation constants. $U(z)$ and $V(z)$ satisfy the differential equations

$$
\left\{\frac{d^{2}}{d z^{2}}+\left[k_{0}^{2}\left(\epsilon(z) / \epsilon_{0}\right)-s^{2}-w^{2}\right]\right\} U(z)=0
$$

and

$$
\left\{\frac{d^{2}}{d z^{2}}-\left(\frac{d \epsilon(z)}{d z}\right) \frac{1}{\epsilon(z)} \frac{d}{d z}+\left[k_{0}^{2}\left(\epsilon(z) / \epsilon_{0}\right)-p^{2}-q^{2}\right]\right\} V(z)=0 .
$$

This equation can be simplified if we take

$$
W(z)=V(z) / \sqrt{\epsilon(z)} .
$$

The wave equation for $W(z)$ then becomes

$$
\left\{\frac{d^{2}}{d z^{2}}+\left[\frac{\left(\epsilon^{1 / 2}\right)^{\prime \prime}}{\epsilon^{2}}+\frac{\epsilon^{\prime}\left(\epsilon^{1 / 2}\right)^{\prime}}{\epsilon^{3}}+\left(k_{0}^{2} \epsilon / \epsilon_{0}-p^{2}-q^{2} \epsilon^{1 / 2}\right)\right]\right\} W=0 .
$$

Now, let us assume that $\epsilon(z)$ is periodic

$$
\epsilon(z)=\epsilon_{0} \epsilon_{r}[1+\eta g(K z)]
$$

where $\eta$ is the modulation coefficient $(\eta<1)$ and $g(K z)$ is a normalized periodic function of period $2 \pi / K$. Then the above differential equations can be written in the form

$$
\frac{d^{2} \psi}{d \xi^{2}}+f(\xi) \psi=0
$$

where

$$
\begin{aligned}
\psi & =U \\
f(\xi) & =k_{0}^{2} \epsilon_{r}[1+\eta g(\xi)]-s^{2}-w^{2}=\sum_{n} a_{n} \exp (\text { in } \xi) \\
\xi & =K z
\end{aligned}
$$

for the TE waves, and

$$
\begin{aligned}
\psi= & W \\
f(\xi)= & \frac{\eta g^{\prime \prime}(\xi)}{2[1+\eta g(\xi)]}-\frac{3}{4}\left[\frac{\eta g^{\prime}(\xi)}{1+\eta g(\xi)}\right] \\
& +\frac{k^{2}}{K^{2}} \epsilon_{r}[1+\eta g(\xi)]-p^{2}-q^{2} \\
= & \sum_{n} b_{n} \exp i n \xi .
\end{aligned}
$$

If the periodic medium is a plasma, then we replace $\epsilon(z)$ by

$$
\epsilon_{0}\left[1-\frac{\omega_{p}^{2}(z)}{\omega^{2}}\right]
$$

where $\omega_{p} / 2 \pi=x$ plasma frequency.

\section{Acoustic Waves}

The one-dimensional equations which govern the acoustic wave propagation in a medium are

$$
\begin{aligned}
\rho \frac{\partial u}{\partial t} & =\frac{\partial T}{\partial z} \\
\frac{\partial s}{\partial t} & =\frac{\partial u}{\partial z} \\
T & =C s
\end{aligned}
$$

where $\rho$ is the mass density, $u$ is the lattice velocity, $s$ is the strain, $T$ is the stress, and $C$ is the elastic constant. The wave equation is simply

$$
\frac{\partial^{2} u}{\partial z^{2}}+\rho \frac{\omega^{2}}{C} u=0 .
$$

If $\rho$ or/and $C$ are periodically modulated, then the above equation will have the same form as (1).

Similar wave equations can be derived for magnetic waves [104].

\section{Electron Waves}

The well-known Schroedinger wave equation for electron waves is

$$
\frac{d^{2} \psi}{d z^{2}}+\frac{8 \pi^{2} m^{*}}{h^{2}}[E-V(z)] \psi=0
$$

which, for the case of a periodic potential $V$, has the same form as (A-14). $E$ is the energy, $h$ is Planck's constant, and $m^{*}$ the effective electron mass.

\section{ACKNOWLEDGMENT}

The work in this paper is the result of many years of involvement and research by the author in the field of periodic structures. During that period the author profited enormously from the encouragement of, and enlightening discussions with, Prof. C. H. Papas (Caltech), Prof. C. Yeh (UCLA), Dr. G. Evans (RDA), Dr. D. Jaggard (Caltech), and Dr. A. Gover (Caltech). The author is most grateful to Prof. S. R. Seshadri (Univ. Wisconsin), Prof. A. C. Scott (Univ. Wisconsin), Prof. K. F. Casey (Kansas State Univ.), Dr. D. Jaggard (Caltech) and Dr. A. Gover (Caltech) for helpful comments on the manuscript. The author gratefully acknowledges the assistance of a number of people at JPL who helped in the literature search, editing, and typing of the manuscript, especially Ms. P. D. Baker and F. Roth.

\section{REFERENCES}

[1] Lord Rayleigh, Phil. Mag., vol. 24, p. 145, 1887.

[2] Kelvin, "Popular lectures," vol. I, p. 185, 1881.

[3] Vincent, Phil. Mag., vol. 46, p. 537, 1898.

[4] M. J. O. Strutt, "Eigenschwingungin einer Saite mit sinusformiger Massenverteilung," Ann. Phys., vol. 85, pp. 129-136, 1928.

[5] B. Van der Pol and M. J. O. Strutt, "On the stability of the solutions of Mathieu's equations," Phil. Mag., vol. 5, pp. 18-38, 1928.

[6] F. Bloch, "Uber die Quantenmechanik der elektronen in kristallgittern," $Z$. Phys., vol. 52, pp. 555-600, 1928.

[7] E. C. Titchmarch, Eigenfunction Expansions A Second Order Differential Equations, Part II. London: Oxford University Press, 1958.

[8] C. Kittel, Introduction to Solid State Physics. New York: Wiley, 1968

[9] G. Allen, "Band structures of one-dimensional crystals with square wells," Phys. Rev., vol. 91, pp. 531-533, 1953.

[10] R. Del. Kronig and W. G. Penny, "Quantum mechanics of 
electrons in crystals," Proc. Roy. Soc. (London), vol. A1 30, pp. 499-513, 1930.

[11] B. Y. Tong, "Electronic structure of one-dimensional binary alloys," Phys. Rev. 1975, pp. 710-722, 1968.

[12] P. Sah and K. P. Srivastava, "A generalized diatomic KronigPenney model," Physics, vol. 43, pp. 528-532, 1969.

[13] R. L. Mooney, "Theory of an efficient interference filter," J. Opt. Soc. Amer., vol. 36, pp. 256-260, 1946.

[14] R. B. Muchmore, "Optimum bandwidth for two layer antireflection films," J. Opt. Soc. Amer., vol. 38, pp. 20-26, 1948.

[15] M. Born and E. Wolf, Principles of Optics. London: Pergamon Press, 1970.

[16] F. Abeles, "Récherches sur la propagation des ondes electromagnétiques sinusoidales dans les milieux stratifiés. Application aux conches minces," Ann. Phys., vol. 5, pp. 596-640 and 706-782, 1950.

[17] N. Brillouin, Wave Propagation in Periodic Structures. New York: Dover Publications, Inc., 1956.

[18] J. R. Pierce, "Theory of the beam-type traveling wave tube," Proc. IRE, vol. 35, pp. 111-123, 1947.

[19] L. M. Field, "Some slow-wave structures for traveling wave tubes," Proc. IRE, vol. 37, pp. 34-40, 1949.

[20] J. R. Pierce, "Circuits for traveling wave tubes," Proc. IRE, vol. 37, pp. 510-515, 1040 .

[21] J. R. Pierce, and N. Wax, "A note on filter type traveling-wave amplifiers," Proc. IRE, vol. 37, pp. 622-625, 1949.

[22] J. R. Pierce, Traveling-Wave Tubes. Princeton, NJ: Van Nostrand, 1950.

[23] S. Sensiper, "Electromagnetic wave propagation on helical conductors," Res. Lab. of Electronics, M.I.T., Tech. Rep. 194, 1951 .

[24] R. M. Bevensee, Electromagnetic Slow Wave Systems. New York: Wiley, 1964.

[25] H. R. Johnson, T. E. Everhart, and A. E. Siegman, "Wave propagation on multiflar helices," IRE Trans. Electron Devices, ED-3, pp. 18-24, 1956 .

[26] P. N. Butcher, "Theoretical study of propagation along tape ladder lines," Proc. Inst. Elec. Eng. (London), Pt. B, vol. 104, pp. 169-176, 1957.

[27] A. A. Oliner and W. Rotman, "Periodic structures in trough waveguides," IRE Trans. Microwave Theory Tech., vol. MTT-7, pp. 134-142, 1959 .

[28] H. S. Kirschbaum and R. Tsu, "A study of a serrated ridge waveguide," IRE Trans. Microwave Theory Tech., vol. MTT-7, pp. 142-148, 1959.

[29] J. C. Simon and V. Biggi, "Un nouveau type d'aerien et son application à la transmission de télévision à grande distance," L'Onde Eléctrique, vol. 332, pp. 883-896, 1954.

[30] J. C. Simon and G. Weill, "Un nouveau type d'antenne à rayonnement longitudinal," Ann. Radioelec., vol. 8, pp. 183-193, 1953.

[31] A. S. Thomas and F. J. Zucker, "Radiation from modulated surface wave structures, I," in IRE Nat. Conv. Rec., pt. I, pp. 153-160, 1957 .

[32] A. A. Oliner and A. Hessel, "Guided waves on sinusoidallymodulated reactance surfaces," IRE Trans. Antennas Propagat., vol. AP.7, pp. 5201-5208, 1959.

[33] A. Hesse1, "General Characteristics of Traveling-Wave Antennas," in Antenna Theory, Part 2, R. E. Collin and F. J. Zucker, Eds. New York: Mc Graw Hill, 1969.

[34] T. Tamir, H. C. Wang, and A. A. Oliner, "Wave propagation in sinusoidally stratified media," IEEE Trans. Microwave Theory Tech., vol. MTT-12, pp. 324-335, 1964.

[35] C. Yeh, K. F. Casey, and Z. Kaprielian, "Transverse Magnetic Wave Propagation in Sinusoidally Stratified Dielectric Media," IEEE Trans. Microwave Theory Tech., vol. MTT-13, pp. 297$302,1965$.

[36] L. R. Lewis and H. Hessel, "Propagation Characteristics of Periodic Arrays of Dielectric Slabs," IEEE Trans. Microwave Theory Tech., vol. MTT-19, pp. 276-286, 1971.

[37] T. Tamir and H. C. Wang, "Scattering of electromagnetic waves by a sinusoidally stratified half-space," Can. J. Phys., vol. 44, pp. 2073-2093, 1966.

[38] K. Casey, J. R. Matthes, and C. Yeh, "Wave propagation in sinusoidally stratified plasma media," J. Math. Phys., vol. 10, pp. 891-897, 1969.

[39] E. S. Cassedy and A. A. Oliner, "Dispersion relations in timespace periodic media, Part 1-Stable Interactions," Proc. IEEE, vol. 52, pp. 1342-1359, 1963.

[40] E. S. Cassedy, "Dispersion relations in time-space periodic media, Part II-Unstable interactions," Proc. IEEE, vol. 55, pp. 1154-1168, 1967

[41] D. E. Holberg and K. S. Kunz, "Parametric properties of fields in a slab of time-varying permittivity," IEEE Trans, Antennas Propagat. vol. AP-14, pp. 183-194, 1966.

[42] R. Rao, "Electromagnetic wave propagation in a dielectric medium space-time modulated by a nonlinear pump wave,"
Proc. IEEE, vol. 56, pp, 1630-1631, 1968.

[43] J. A. Kong and L. H. Wang, "Dispersion analysis of optoacoustic interactions in moving media," J. Appl. Phys., vol. 43, pp. 1396-1400, 1972 .

[44] C. Elachi, "Electromagnetic wave propagation and wave-vector diagram in space-time periodic media," IEEE Trans. Antennas Propagat., vol. AP-20, pp. 534-536, 1972.

[45] - "Electromagnetic wave propagation and source radiation in space-time periodic media," California Inst. Technol., Pasadena, Antenna Lab. Rep. 61, 1971.' (Contains many references on this subject.)

[46] K. Casey, C. Yeh, and Z. Kaprielian, "Cerenkov radiation in inhomogeneous periodic media," Phys. Rev., vol. 140, pp. B768-B775, 1965 .

[47] K. Casey, "Cerenkov radiation in a periodic laminar dielectric," Can. J. Phys., vol. 46, pp. 1957-1966, 1968.

[48] -, "Dipole radiation in a periodically stratified medium," Canad. J. Phys., vol. 46, pp. 2543-2551, 1968.

[49] K. Casey and C. Yeh, "Transition radiation in periodically stratified plasma," Phys. Rev., A, vol. 2, pp. 810-818, 1970.

[50] B. Singer and T. Tamir, "Diffraction of light from a filimentary source by parallel acoustic wave fronts," J. Opt. Soc. Amer. vol. 60, pp. 1640-1653, 1970 .

[51] C. Elachi, "Dipole antenna in space-time periodic media," IEEE Trans. Antennas Propagat., vol. AP-20, pp. 280-287, 1972.

[52] - "Cerenkov and transition radiation in space-time periodic media,"J. Appl. Phys., vol. 43, pp. 3719-3723, 1972.

[53] R. M. White, "Surface elastic waves," Proc. IEEE, vol. 58, pp. 1238-1275, 1970.

[54] H. Kogelnik and C. V. Shank, "Stimulated emission in a periodic structure," Appl. Phys. Letts., vol. 18, pp. 152-154, 1971

[55] C. V. Shank, J. E. Bjorkholm, and H. Kogelnik, "Tunable distributed feedback dye laser," Appl. Phys. Lett., vol. 18, pp. 395-396, 1971.

[56] H. Kogelnik and C. V. Shank, "Coupled-wave theory of distributed feedback lasers," J. Appl. Phys., vol. 43, pp. $2327-$ $2335,1972$.

[57] K. O. Hill and A. Watanabe, "A distributed feedback side coupled laser," Opt. Comm., vol. 5. Dp. 389-393. 1972.

[58] R. L. Fork, K. R. German, and E. A. Chandross, "Photodimer distributed feedback laser," Appl. Phys. Lett., vol. 20, pp. $139-141,1972$.

[59] J. F. Bjorkholm and C. V. Shank, "Higher order distributed feedback oscillators," Appl. Phys. Lett., vol. 20, pp. 306-308, 1972 .

[60] Periodic Modulation of the Boundary for DFB Structures as proposed by Kogelnik and Shank [56], A. Yariv and, indepen dently, by S. Wang in the VII International Quantum Electronic Conference, Montreal, (1972), and by P. Zory in the Topical Meeting on Integrated Optics, Las Vegas, NE (1972). This structure was analyzed by $W$. Wang, "Proposal of periodic layered waveguide structures for distributed lasers," J. Appl. Phys., vol. 44, pp. 767-780, 1973.

[61] D. P. Schinke, R. G. Smith, F. G. Spencer, and M. F. Gavin "Thin film distributed feedback laser fabricated by ion milling," Appl. Phys. Lett., vol. 21, pp. 494-496, 1972.

[62] D. Marcuse, "Hollow dielectric waveguide for distributed feedback lasers," IEEE J. Quentum Electron., vol. QE-8, pp. 661 669,1972 .

[63] P. Zory, "Laser oscillation in leaky corrugated optical waveguides," Appl. Phys. Lett., vol. 22, pp. 125-128, 1973.

[64] J. E. Bjorkholm, T. P. Sosnowski, and C. V. Shank, "Distributed feedback lasers in optical waveguides deposited on anisotropic substrate," Appl. Phys. Lett., vol. 22, pp. 132-134, 1973.

[65] H. Kogelnik, C. V. Shank, and J. E. Bjorkholm, "Hybrid scattering in periodic waveguides," Appl. Phys. Lett., vol. 22, pp. 135137,1973 .

[66] S. Wang and S. Sheem, "Two-dimensional distributed feedback lasers and their applications," Appl. Phys. Lett., vol. 22, pp. $460-462,1973$.

[67] M. Nakamura, A. Yariv, H. W. Yen, S. Somekh, and H. L. Garvin, "Optically pumped GaAs surface laser with corrugation feedback," Appl. Phys. Lett., vol. 22, pp. 515-516, 1973.

[68] R. E. DeWaines and W. F. Hall, "Conditions for laser oscilla tions in distributed feedback waveguides," Appl. Phys. Lett., vol. 23, pp. 28-30, 1973 .

[69] M. Nakamura, H. W. Yen, A. Yariv, E. Garmire, S. Somekh, and $H$. L. Garvin, "Laser oscillation in epitaxial GaAs waveguides with corrugated feedback," Appl. Phys. Lett., vol. 23, pp. $224-225,1973$.

[70] C. Elachi and C. Yeh, "Periodic structures in integrated optics," J. Appl. Phys., vol. 44, pp. 3146-3152, 1973.

[71] $S$. R. Chinn, "Effects of mirror reflectivity in a distributed feed. back laser," IEEE J. Ouantum Electron., vol. QE-9, pp. 574 $580,1973$.

[72] M. Nakamura and A. Yariv, "Analysis of the threshold of double 
heterojunction GaAs-GaAlAs laser with a corrugated surface," Opt. Commun., vol. 11, pp. 18-20, 1974.

[73] S. Wang, R. F. Cordero, and C. C. Tseng, "Analysis of DFB and DBR laser structures by method of multiple reflections," $J$. Appl. Phys., vol. 45, pp. 3975-3977, 1974.

[74] R. F. Cordero and S. Wang, "Threshold condition for thinfilm DFB lasers," Appl. Phys. Lett., vol. 24, pp. 474-476, 1974.

[75] S. Wang, "Principles of distributed feedback and distributed Bragg reflector lasers," IEEE J. Quantum Electron., vol. QE-10, pp. 413-426, 1974.

[76] W. S. C. Chang, "Periodic Structures and Their Application in Integrated Optics," IEEE Trans. Microwave Theory Tech., vol. MTT-21, pp. 775-785, 1974.

[77] $\mathrm{S}$. Wang, "Thin film bragg lasers for integrated optics," Wave Electronics, vol. 1, pp. 31-59, 1974.

[78] S. R. Chinn and P. L. Kelley, "Analysis of the transmission, reflection, and noise properties of distributed feedback laser amplifiers," Opt. Commun., vol. 10, pp. 123-126, 1974.

[79] R. Schubert, "Theory of optical waveguide distributed lasers with nonuniform gain and coupling," J. Appl. Phys., vol. 45, pp. 209-215, 1974

[80] H. W. Yen, M. Nakamura, E. Garmire, S. Somekh, A. Yariv, and H. L. Garvin, "Optically pumped GaAs waveguide lasers with a fundamental $0.11 \mu$ corrugation feedback," Opt. Commun., vol. 9, pp. 35-37, 1973.

[81] A Yariv and H. W. Yen, "Bragg amplification and oscillation in periodic optical media," Opt. Commun., vol. 10, pp. 120$122,1974$.

[82] C. V. Shank, R. V. Schmidt, and B. I. Miller, "Double heterostructure GaAs distributed feedback laser," Appl Phys. Lett., vol. 25, pp. 200-201, 1974.

[83] D. R. Scifres, R. D. Burnham, and W. Streifer, "Distributed feedback single heterojunction GaAs diode laser," Appl. Phys. Lett., vol. 25, pp. 203-206, 1974.

[84] M. Nakamura, K. Aiki, J. I. Umeda, A. Yariv, H. W. Yen, and T. Morikawa, "GaAs-Ga ${ }_{1} \mathrm{Al}_{x} \mathrm{As}$ double heterostructure distributed feedback lasers," Appl. Phys. Lett., vol. 25, pp. 487-488, 1974 .

[85] H. M. Stoll and D. H. Seib, "Distributed feedback GaAs hemojunction injection laser," Appl. Opt., vol. 13, pp. 1981-1982, 1974.

[86] D. B. Anderson, R. R. August, and J. E. Coker, "DFB double heterostructure injection laser with fundamental grating," Appl. Opt., vol. 13, pp. 2742-2744, 1974.

[87] R. A. Fisher, "Possibility of a distributed feedback X-ray laser," Appl. Phys. Lett., vol. 24, pp. 598-599, 1974.

[88] A. Yariv, "Analytical considerations of bragg coupling coefficients and distributed feedback X-ray lasers in single crystals," Appl. Phys. Lett., vol. 25, pp. 105-107, 1974.

[89] C. Elachi, G. Evans, and F. Grunthaner, "Proposed DFB crystal cavities for X-ray lasers," Appl. Opt., vol. 14, pp. 14-14, 1975.

[90] C. Elachi, G. Evans, and C. Yeh, "Transversely bounded DFB lasers," J. Opt. Soc. Amer., vol. 65, pp. 404-412, 1975.

[91] W. Streifer, R. D. Burnham, and D. R. Scifres, "Effect of external reflectors on longitudinal modes of DFB lasers," IEEE $J$. Quantum Electron., vol. QE-11, pp. 154-161, 1975.

[92] A. Yariv and A. Grover, "Equivalence of the coupled mode and Floquet-Bloch Formalism in periodic optical waveguides," Appl. Phys. Lett., vol. 26, pp. 537-539, 1975.

[93] D. R. Scifres, R. D. Burnham, and W. Streifer, "Highly collimated laser beams from electrically pumped SH GaAs/GaAlAs DFB lasers," Appl. Phys. Lett., vol. 26, pp. 48-50, 1975.

[94] F. K. Reinhart, R. A. Logan, and C. V. Shank, "GaAs$\mathrm{Al}_{x} \mathrm{Ga}_{1} x^{\mathrm{As}}$ injection lasers with distributed Bragg reflectors," Appl. Phys. Lett., vol. 27, pp. 45-48. 1975.

[95] H. C. Casey, S. Somekh, and M. Ilegems, "Room temperature operation of low threshold separate confinement heterostructure injection laser with distributed feedback," Appl. Phys. Lett., vol. 27, pp. 142-144, 1975.

[96] K. Aiki, M. Nakamura, J. Umeda, A. Yariv, A. Katzir, and H. W. Yen, "GaAs-GaAlAs distributed feedback diode lasers with separate optical and carrier confinement," Appl. Phys. Lett., vol. 27, pp. 145-146, 1975.

[97] S. Wang and W.T. Tsang, "Analysis of ring distributed feedback lasers,"J. Appl. Phys., vol. 45, pp. 3978-3980, 1974.

[98] A. Yariv, "Coupled Mode Theory of Guided Wave Optics," IEEE J. Quantum Electron., vol. QE-9, pp. 919-933, 1973.

[99] N. Bloembergen and A. J. Sievers, "Nonlinear optical properties of periodic laminar structures," Appl. Phys. Lett., vol. 17 pp. 483-485, 1970

$[100] \mathrm{S}$. Somekh and A. Yariv, "Phase matching by periodic modulation of the nonlinear optical properties," Opt. Commun., vol. 6, pp. 301 -304, 1972.

[101] S. Somekh and A. Yariv, "Phase matchable nonlinear optical interactions in periodic thin films," Appl. Phys. Lett., vol. 21, pp. 140-141, 1972 .
[102] C. L. Tang and P. L. Bey, "Phase matching in second harmonic generation using artificial periodic structures," IEEE Quantum Electron., vol. QE-9, pp. 9-17, 1973.

[103] B. F. Levine, C. G. Bethea, and R. A. Logan, "Phase-matched second harmonic generation in a liquid filled waveguide," Appl. Phys. Lett., vol. 26, pp. 375-377,1974.

[104] C. Elachi, "Magnetic wave propagation in periodic media," IEEE Trans Magn., vol. MAG-11, pp. 36-39, 1975.

[105] C. Elachi, "Distributed feedback acoustic surface wave oscillator," IEEE Trans. Microwave Theory Tech., vol. MTT-22, pp. $907-908,1974$.

[106] C. Elachi, "Acoustic microwave generation in a periodic piezoelectric medium with drifting charges," J. Appl. Phys., vol. 5, pp. 159-164, 1974.

[107] A. Gover and A. Yariv, "Monolithic solid state traveling wave amplifier,"J. Appl. Phys., vol. 45, pp. 2596-2600, 1974.

[108] J. Metner and F. W. Schafke, Mathieusche Funktionen und Spheroidfunktionen. Berlin: Springer-Verlag, 1954.

[109] M. J. O. Strutt, Lamesche-Mathieusche und Verwandte Funktionen. Berlin: Springer-Verlag, 1932, (the numbers Strutt uses to label the $E$ axis in Fig. 4 are too large by a factor of 4).

[110] W. Shockley, "On the surface states associated with a periodic potential," Phys. Rev., vol. 56, pp. 317-323, 1939.

[111] D. Marcuse, Light Transmission Optics. New York: Van Nostrand, 1972.

[112] J. R. Pierce, Almost All About Waves. Cambridge, MA: M.I.T. Press, 1974. Also see J. R. Pierce, "Coupling of modes of propagation," J. Appl Phys., vol. 25, pp. 179-183, 1954.

[113] R. S. Chu and T. Tamir, "Guided wave theory of light diffraction by acoustic microwave," IEEE Trans. Microwave Theory Tech., vol. MTT-17, pp. 486-504, 1970.

[114] S. F. Su and T. K. Gaylord, "Calculation of arbitrary order diffraction efficiencies of thick gratings with arbitrary grating shape,"J. Opt. Soc. Am., vol. 65, pp. 59-64, 1975.

[115] D. Jaggard and C. Elachi, "Coupled waves theory for higher order bragg scattering in periodic media," submitted to J. Opt. Soc. Am., vol. 66, pp. 674-682, 1976.

[116] B. Minakovic and J. Gokgor, "Attenuation and phase shift coefficients in dielectric loaded periodic waveguides," IEEE Trans. Microwave Theory Tech., vol. MTT-21, pp. 568-568, 1973.

[117] D. J. Mead, "A general theory of harmonic wave propagation in linear periodic systems with multiple coupling," J. Sound Vibr., vol. 27, pp. 235-260, 1973.

[118] G. Sen Gupta, "Natural flexural waves and the normal modes of periodically supported beams and plates," J. Sound Vibr., vol. 13, pp. 89-101, 1970 .

[119] S. T. Peng, T. Tamir, and H. L. Bertoni, "Theory of periodic dielectric waveguides," IEEE Trans. Microwave Theory Tech., vol. MTT-23, pp. 123-133, 1975.

[120] A. Hessel and A. A. Oliner, "Mode coupling regions in the dispersion curves of modulated slow wave Antennas," in PTGAP Int. Symp. Digest., pp. 104-108, 1963.

[121] H. C. Wang, "Electromagnetic wave propagation along a sinusoidally stratified dielectric slab," Polytechnic Institute of Brooklyn, Brooklyn, NY, Rep. PIBMRI-1227, 1964.

[122] A. A. Oliner, "Guided complex waves on slow-wave periodic structures," in Electromagnetic Wave Theory, J. Brown, ed., Pt. 1, New York: Pergamon Press, 1967, p. 467.

[123] C. Elachi and C. Yeh, "Mode conversion in periodically disturbed thin-film waveguides," J. Appl. Phys., vol. 45, pp. 3494-3499, 1974.

[124] C. Elachi, G. Evans, and C. Yeh, "Fiber and diffused waveguide structures for distributed feedback lasers," IEEE Trans. Microwave Theory Tech., vol. MTT-23, pp. 532-534, 1975.

[125] D. Marcuse, "Light scattering from periodic refractive index fluctuations in asymetric slab waveguides," IEEE J. Quantum Electron., vol. QE-11, pp. 162-168, 1975.

[126] F. W. Dabby, A. Kestenbaum, and V. C. Peak, "Periodic dielec. tric waveguides," Opt. Commun., vol. 6, pp. 125-130, 1972.

[127] F. W. Dabby, M. A. Saifi, and A. Kestenbaum, "High frequency cutoff periodic dielectric waveguides," Appl. Phys. Lett., vol. 22, pp. 190-191, 1973 .

[128] T. Tamir, "Scattering of electromagnetic waves by a sinusoidally stratified half-space: Part II," Can. J. Phys., vol. 44, pp. 24612494, 1966.

[129] A. Hessel and A. A. Oliner, "A new theory of wood's anomalies on optical gratings," Appl. Opt., vol. 4, pp. 1275-1297, 1965.

[130] G. Borrmann, Trends in Atomic Physics, $O$. R. Frisch et al., eds., New York: Interscience Publishing, Inc., 1959.

[131] B. W. Batterman and $H$. Cole, "Dynamic diffraction of X-rays by perfect crystals," Rev. Mod. Phys., vol. 36, pp. 681-717, 1964.

[132] C. Elachi, D. Jaggard, and C. Yeh, "Transients in a periodic slab: Coupled waves approach," IEEE Trans. Antennas Propagat. vol. AP-23, pp. 352-356, 1975.

[133] Lord Rayleigh, "On the dynamical theory of gratings," Proc. 
Roy. Soc., ser. A, vol. 79, pp. 399-416, 1907.

[134] N. Neviere, R. Petit, and M. Cadilhac, "About the theory of optical grating coupler-waveguide systems," Opt. Commun., vol. 8, pp. 113-117, 1973.

[135] M. Neviere, P. Vincent, R. Petit, and M. Cadilhac, "Systematic study of resonance of holographic thin film," Opt. Commun., vol. 9, pp. 48-53, 1973.

[136] M. Neviere, "Determination of the coupling coefficient of a holographic thin film coupler," Opt. Commun., vol. 9, pp. 240-245, 1973.

[137] R. Petit and M. Cadilhac, "Sur al diffraction d'une onde plane par un reseau infiniment conducteur," C. R. Acad. Sci. Paris, ser. B262, pp. 268-471, 1966.

[138] R. F. Millar, "On the Rayleigh Assumption in scattering by a periodic surface," Proc. Cambridge Phil. Soc., vol. 65, pp. 773-790, 1969, and vol. 69, pp. 217-225, 1971

[139] S. T. Peng, H. L. Bertoni, and T. Tamir, "Analysis of periodic thin film structures with rectangular profile," Opt. Commun., vol. 10, pp. $91-94,1974$.

[140] L. L. Hope, "Theory of optical grating couplers," Opt. Commun., vol. 5, pp. 179-182, 1972.

[141] K. Sakuda and A. Yariv, "Analysis of optical progagation in a corrugated dielectric waveguide," Opt. Commun., vol. 8, pp. $1-4,1973$.

[142] K. Ogawa, W. S. C. Chang, B. L. Sopori, and F. J. Rosenbaum "A theoretical analysis of etched grating couplers for integrated optics," IEEE J. Quantum Electron., vol. QE-9, pp. 29-42, 1973.

[143] D. Marcuse and R. Derosier, "Mode conversion caused by diameter changes of a round dielectric waveguide," Bell Syst. Tech. J., vol. 98, pp. 3217-3242, 1969.

[144] J. Chandezon, G. Comet, and G. Raoult, "Propagation des modes dans les guides cylindrigués a génératrice sinusoidale. Expression champs," C. R. Acad. Sci. Paris, ser. B, vol. 277, pp. $355-358$ and $403-405,1973$.

[145] O. R. Asfar and A. H. Nayfch, "Circular waveguide with sinusoidally perturbed walls," IEEE Trans. Microwave Theory Tech., vol. 23, pp. 728-734, 1975.

[146] P. C. Waterman, "Scattering by periodic surfaces," J. Acoust. Soc. Amer., vol. 57, pp. 791-802, 1975.

[147] J. F. Carison and A. E. Heins, "The reflection of an electromagnetic plane wave by an infinite set of plates $1, " Q$. Appl. Math., vol. 4, pp. 313-329, 1947.

[148] - "The reflection of an electromagnetic plane wave by an infinite set of plates II," Q. Appl. Math., vol. 5, pp. 82-88, 1947.

[149] F. Berz, "Reflection and refraction of microwaves at a set of parallel metallic plates," Proc. Inst. Elec. Eng., vol. B98, pp. 47-55, 1951.

[150] E. A. N. Whitehead, "The theory of parallel-plate media for microwave lenses," Proc. Inst. Elec. Eng., vol. B98, pp. 133140,1951 .

[151] J. A. Desanto, "Scattering from a periodic corrugated structure: Thin comb with soft boundaries," J. Math. Phys., vol. 12, pp. 1913-1923, 1971.

[152] - "Scattering from a periodic corrugated structure: Thin comb with hard boundaries," J. Math. Phys., vol. 13, pp. $336-341,1972$.

[153] -, "Scattering from a periodic corrugated surface: Semiinfinite alternately filled plates," J. Acoust. Soc. Amer., vol. 53, pp. 714'-734, 1973.

[154] M. A. Isakovitch, Akust. Z., vol. 3, pp. 37-45, 1957. (Paper in Russian.)

[155] J. S. Samuels, "On propagation of waves in slightly rough ducts," J. A coust. Soc. Am., vol. 31, pp. 319-325, 1959.

[156] R. F. Salant, "Acoustic propagation in waveguides with sinusoidal walls," J. Acoust. Soc. Amer., vol. 53, pp. 504-507, 1973.

[157] A. H. Nayfeh, "Sound waves in two dimensional ducts with sinusoidal walls," J. Acoust. Soc. Amer., vol. 56, pp. 768 $770,1974$.

[158] A. H. Nayfeh, "Acoustic waves in ducts with sinusoidally perturbed walls and mean flow," J. Acoust. Soc. Amer., vol. 57, pp. 1036-1039, 1975.

[159] G. R. Valenzuela, H. B. Laing, and J. C. Daley, "Ocean spectra for the high frequency waves as determined from airborne radar measurements," J. Mar, Res., vol. 29, pp. 69-84, 1971.

[160] G. L. Tyler, W. F. Faulkerson, A. L. Peterson, and C. C. Teague, "Second order scattering from the sea: $10 \mathrm{~m}$ radar observation of the doppler continuum," Science, vol. 177, pp. 349-351, 1972 .

[161] D. E. Barrick, "First order theory and analysis of MF/HF/VHF scatter from the seg," IEEE Trans. Antennas Propagat., vol. AP-20, pp. 2-10, 1972

[162] P. Beckman and A. Spizzichino, The Scattering of Electro. magnetic Waves from Rough Surfaces. Oxford: Pergamon Press, 1963.
[163] H. H. Kuchl, "Electromagnetic radiation from an electric dipole in a cold anisotropic plasma," Phys. Fluids, vol. 5, pp. 1094-1103,1962.

[164] S. J. Smith and E. M. Purcell, "Visible light from localized surface charges moving across a grating," Phys. Rev., vol. 92, pp. 1069-1069, 1953.

[165] G. Toraldo Di Francis, "On the theory of some Cerenkov effects," Nuovo Cimento, vol. 16, pp. 61-77, 1960.

[166] A. Hessel, "Resonances in the Smith-Purcell effect," Can.J. Phys., vol. 42, pp. 1195-1211, 1969.

[167] D. A. Hill and J. R. Wait, "Scattering of a transient plane wave by a periodic grating," Radio Sct., vol. 6, pp. 1003-1009, 1971.

[168] C. V. Shank and R. V. Schmidt, "Optical technique for producing $0.1 \mu$ periodic surface structures," Appl. Phys. Lett., vol. 23, pp. 154-155, 1973.

[169] A. Yariv and D. R. Armstrong, "Travelling wave interaction in the optical region: A theoretical examination," J. Appl. Phys., vol. 44, pp. 1664-1665, 1973.

[170] L. Solymar and E. A. Ash, "Some traveling wave interaction in semiconductor theory and design considerations," Int. J. Electron., vol. 20, pp. 127-148, 1966.

[171] M. Sumi, "Traveling Wave Amplification by Drifting Carriers in Semiconductors," Japan J. Appl. Phys., vol. 6, pp. 688-698, 1967.

[172] M. Meyer and T. Van Duzer, "Traveling wave amplification and power flow in conducting solids," IEEE Trans. Eelectron De. vices, vol. ED-17, pp. 193-199, 1970.

[173] M. Sumi and T. Suzuki, "Evidence for directional coupling between semiconductor carriers and slow circuit waves," $A p p l$. Phys. Lett., vol. 13, pp. 326-327, 1968.

[174] A. Gover and A. Yariv, "Intraband radiative transitions and plasma-electromagnetic wave coupling in periodic semiconduc tor structure," to be published in J. Appl. Phys.

[175] A. R. Panicoli, Y. T. Lo, and G. A. Deschamps, "A reflector antenna corrected for spherical, coma and chromatic aberra. tions," Proc. IEEE, vol. 59, pp. 311-31 2, 1971.

[176] S. W. Lee and T. T. Fong, "Electromagnetic wave scattering from an active corrugated structure," J. Appl. Phys., vol. 43 pp. 388-395, 1972.

[177] D. C. Flanders, H. Kogelnik, R. V. Schmidt, and C. V. Shank, "Grating filter for thin film optical waveguides," Appl. Phys. Lett., vol. 24, pp. 194-196, 1974. Also see R. V. Schmidt D. C. Flanders, C. V. Shank, and R. D. Standley, "Narrow band grating filters for thin film optical waveguides," Appl. Phys. Lett., vol. 25, pp. 651-652, 1974 .

[178] M. S. Chang, "Tolerance to the phase-matching condition for the Integrated acousto-optic filter," Appl. Opt., vol. 13, pp. 1867-1871, 1974.

[179] T. Tamir and H. L. Bertoni, "Lateral displacement of optical beams at multilayered and periodic structures," J. Opt. Soc. Amer., vol. 61, pp. 1397-1413,1971.

[180] J. H. Harris, R. K. Winn, and D. G. Dalgoutte, "Theory and design of periodic couplers," Appl. Opt., vol. 11, pp. 2234 2241, 1972 .

[181] S. T. Peng, T. Tamir, and H. L. Bertoni, "Leaky wave analysis of optical periocic couplers," Electron. Lett., vol. 9, pp. 150-152, 1973.

[182] L. Kuhn, P. F. Heidrich, and E. G. Lean, "Optical guided wave mode conversion by an acoustic surface wave," Appl Phys. Lett., vol. 19, pp. 428-430, 1971.

[183] H. Sasaki, J. Kushibiki, and N. Chubachi, "Efficient acoustooptic TE-TM mode conversion in ZnO films," Appl. Phys. Lett. vol. 25 , pp. $476-477,1974$

[184] Y. Ohmachi, "Acousto-optic TE-TM mode conversion in a thin film of amorphous tellurium dioxide," Electron. Lett., vol. 9 pp. 539-541, 1973 .

[185] S. C. C. Tseng, A. R. Reisinger, E. A. Geiss, and C. G. Powell, "Mode conversion in magneto-optic waveguides subjected to a periodic permalloy structure," Appl. Phys. Lett., vol. 24, pp. 265-267, 1974.

[186] D. P. Gia Russo and J. H. Harris, "Electro-optic modulation in a thin film waveguide," Appl. Opt., vol. 10, pp. 2786-2788, 1971 .

[187] J. N. Polky and J. H. Harris, "Electro-optic thin film modulator," Appl. Phys. Lett., vol. 21, pp. 307-309, 1972.

[188] P. K. Tien, R. J. Martin, R. Wolfe, R. C. LeCraw, and S. L. Blank, "Switching and modulation of light in magneto-optic waveguides of garnet films," Appl Phys. Lett., vol. 21, pp. 294-396, 1972 .

[189] L. Kuhn, M. L. Dakss, P. F. Heidrich, and B. A. Scott, "Deflection of an optical guided wave by a surface acoustic wave," Appl. Phys. Lett., vol. 17, pp. 265-267, 1970.

[190] T. G. Giallorenzi and A. F. Milton, "Light deflection in multimode waveguides using the acousto-optic interaction," J. Appl. Phys., vol. 45, pp. 1762-1774, 1974

[191] D. A. Wille and M. C. Hamilton, "Acousto-optic deflection in 
$\mathrm{Ta}_{2} \mathrm{O}$, waveguides," Appl. Phys. Lett., vol. 24, pp. 159-160, 1974 .

[192] $M$. Luukkala and $P$, Merilainen, "Image scanning by acoustoelectro-optic interaction," Elect. Lett., vol. 10, pp. 80-81, 1974.

[193] P. K. Cheo and T. M. Reeder, "Acousto-optical interaction of a 10.6 4 guided wave mode in GaAs thin film waveguide," Presented at the Spring Meeting of Opt. Soc. Amer., 1973.

[194] C. J. Kramer and P. Das, "Light diffraction by ultrasound in a liquid waveguide," Proc. Soc. Photo.Opt. Inst. Eng., vol. 38, pp. 117-123,1973.

[195] J. M. White, P. F. Heidrich, and E. G. Lean, "Thin film acoustooptic interaction in $\mathrm{LiNbO}_{3}, "$ Electron. Lett., vol. 10, pp. 510-511, 1974 .

[196] M. Kondo, Y. Ohta, F. Saito, H. Sasaki, N. Chubachi, and K. Koizumi, "Acousto optic diffraction by an acoustic surface wave in an ion exchanged waveguide," Electron Lett., vol. 10, pp. 518-519, 1974 .

[197] C. Elachi and C. Yeh, "Frequency selective coupler for integrated optic systems" Opt. Commun., vol. 7, pp. 201-204, 1973.

[198] R. M. White and F. W. Voltmer, "Direct piezoelectric coupling to surface elastic waves," Appl. Phys. Lett., vol. 1, pp. 314-316, 1965.

[199] G. A. Coquin and H. F. Tiersten, "Detection of piezoelectric surface waves," J. Amer. Acoust. Soc., vol. 41, pp. 921-939, 1967.

[200] S. G. Joshi and R. M. White, "Excitation and detection of surface elastic waves in piezoelectric crystals," J. Amer. Acoust. Soc., vol. 46, pp. 17-27, 1969.

[201] J. D. Maines and E. G. S. Paige, "Surface acoustic wave components, devices, and applications," Proc. IEEE, vol. 120, pp. 1078-1110,1973.

[202] D. H. Hurlburt, "Acoustic surface wave excitation in layered structures," IEEE Trans. Sonics Ultrason., vol. 20, pp. 331-334, 1973.

[203] H. L. Bertoni, "Piezoelectric Rayleigh wave excitation by bulk wave scattering," IEEE Trans. Microwave Theory Tech., vol. MTT-17, pp. 873-882, 1969.

[204] B. A. Auld and S. A. Farnow, "Coupling of surface and volume acoustic waves by a photoconductive grating," J. Appl. Phys., vol. 45, pp. 4314-4319, 1974 .

[205] O. W. Ot to and R. D. Weglein, "Scattering of acoustic surface waves from periodic grating filters," Electron Lett., vol. 10, pp. 383-384, 1974

[206] J. Melngailis and G. T. Flynn, "16-channel surface acoustic wave grating filter bank for real time spectral anal yses," Electron. Lett., vol. 10, pp. 107-109, 1974.

$[207]$ R. D. Weglein and $O$. W. Otto, "Characteristics of periodic acoustic surface wave grating filters," Electron. Lett., vol. 10, pp. 68-69, 1974.

[208] R. H. Tancrell, "Analytic design of surface wave bandpass filters," IEEE Trans. Sonics Ultrason., vol. SU-21, pp. 12-22, 1974.

[209] R. C. Williamson and H. I. Smith, "Use of surface elastic wave reflection gratings in large time-bandwidth pulse compression filters," IEEE Trans. Microwave Theory Tech., vol. MTT-21, pp. $195-205,1973$.

[210] K. Blotekjaer, K. A. Ingebrigtsen, and H. Skeie, "A method for analyzing waves in structures consisting of metal strips on dispersive media," IEEE Trans. Electron Devices, vol. ED-20, pp. $1133-1138,1973$. See also "Acoustic surface waves in piezoelectric materials with periodic metal strips on the surface," Proc. IEEE, vol. 58, pp. 1139-1146, 1970.

[211] Lord Rayleigh, Theory of Sound. Vol. 2, New York: Dover Publishing, Inc., 1945, p. 390.

[212] W. E. Kack, "Pulse compression with periodic gratings and zone plate gratings," Proc. IEEE, vol. 58, pp. 1395-1396, 1970.

[213] H. Eachler, G. Saljc, and H. Stahl, "Thermal diffusion measurements using spatially periodic temperature distributions induced by laser light," J. Appl. Phys., vol. 44, pp. \$383-5388, 1973.

[214] W. R. Klein and B. D. Cook, "Unified approach to ultrasonic light diffraction," IEEE Trans. Sanics Ultrason., vol. SU-14, pp. 123-134, 1967 .

[215] E. Conwell, "Theory of second harmonic generation in optical waveguides," IEEE Quantum Electron. J., vol. QE-9, pp. 867$879,1973$.

[216] S. E. Harris, S. T. K. Nich, and D. K. Winslow, "Electronically tunable acousto-optic fulter," Appl. Phys. Lett., vol. 15, pp. $325 \sim 327,1969$.

[217] S. E. Harris, S. T. K. Nich, and R. S. Feigelson, "CaMoO, electronically tunable optical filter," Appl. Phys. Lett., vol. 17, pp. 223-225, 1970 .

[218] R. Ulrich and $M$. Take, "Submillimeter waveguiding on periodic metallic structures," Appl. Phys. Lett., vol. 22, pp. 251-253, 1973.

[219] S. W. Lee and W. R. Jones, "Surface waves on two-dimensional corrugated structures," Radio Scl, vol. 6, pp. $811-818,1971$.
[220] P. B. Rhines, "Wave propagation in a periodic medium with application to the ocean,"J. Geophys. Space Phys., vol. 8, pp. $303-319,1970$.

[221] T.H. Goldsmith, "The Visual System of Insects," The Physiology of Insecta, M. Rockstein, ed., New York: Academic Press, 1964.

[222] C. G. Bernhard, ed., The Functional Organization of the Com. pound Eye, London: Pergamon Press, 1966.

[223] W. H. Miller, G. D. Bernard, and J. L. Allen, "The optics of insect compound eyes," Science, vol. 162, pp. 760-767, 1968.

[224] G. D. Bernard and W. H. Miller, "Interference filters in the cornea of diptera," Investigative Ophthatmology, vol. 7, pp. $416-434,1968$

[225] W. H. Miller and G. D. Bernard, "Butterfly glow," J. Ultrastructure Res., vol. 24, pp. 286-294, 1968.

[226] G. D. Bernard and W. H. Miller, "What does antenna engineering have to do with insect eyes," IEEE Student J., pp. 2-8, Jan, 1970 .

[227] H. deVties, "Rotatory power and other optical properties of certain liquid crystals," Acta Crystallog, vol. 4, pp. 219-226, 1951 .

[228] J.H. Conners, "Electromagnetic wave propagation in cholesteric materials," J. Opt. Soc. A mer., vol. 58, pp. 875-879, 1968.

[229] C. Elachi and C. Yeh, "Stop bands for optical wave propagation in cholesteric liquid crystals," J. Opt. Soc. Amer., vol. 63, pp. 840-842, 1973.

[230] T. Kallard, ed, Liquid Crystals and Their Applications, New York: Optosonic Press, 1970.

[231] J, Adams, W, Hass, and J, Dailey, "Cholesteric films as optical filters," J. Appl. Phys, vol, 42, pp. 4096-4098, 1971.

[232] J. L. Ferguson, "Cholesteric structure, optical properties," Mol. Cryst. Liq. Cryst., vol. 1, pp. 293-307, 1966.

[233] D. Taupin, "Etude quantitative theorique des reflexions selectives de la lumiere par les cholesteriques parfaits," $J$. Phys., vol. $30, \mathrm{pp}, \mathrm{C} 4, \mathrm{C} 32,1969$.

[234] D. W. Berreman and T. J. Scheffer, "Bragg reflection of light from single domain cholesteric liquid crystal films," Phys. Rev. Lett., vol. 25 , pp. 577-581, 1970 .

[235] F. I. Kats, "Optical properties of cholesteric liquid crystals," Sov. Phys.-JETP, vol. 32, pp. 1004-1007, 1971.

[236] $R_{*}$ Dreher, G. Meier, and A. Saupe, "Selective reflection by cholesteric liquid crystals," Mol. Cryst. Liq. Cryst., vol. 13, pp. $17-26,1971$.

[237] A. S. Marathay, "Propagation of polarized light in a cholesteric structure," Opt. Commun., Vol. 3, pp. 369-373, 1971.

[238] E. I. Kats, "Cerenkov radiation in cholesteric liquid crystals," Sov. Phys.JETP, vol. 34,pp. 899-901, 1972.

[239] J.W. Sheldon and $Y$. R. Shen, "Umklapp optical third harmonic generation in cholesteric liquid crystals," Phys. Rev. Lett., vol. 26 , pp. 538-541, 1971 .

[240] L. Cremer and H. O. Leilich, "Zur theorie der biegekettenleiter," Archiv der Elektrischen Ubertragung, vol. 7, pp. 261-270, 1953.

[241] M. A. Heckel, "Investigations on the vibrations of grillages and other simple beam structures," J. Acoust. Soc. Amer., vol, 36, pp. $1335-1343,1964$.

[242] D. J, Mead, "The random vibrations of a multisupported heavily damped beam," Shock Vibr. Bull., vol. 35, pp. 45-54, 1966.

[243] D. J. Mead, "Free wave propagation in periodically supported infinite beams," $J$, Sound Vibr, vol, 11, pp. 181-197, 1970.

[244] D. J. Mead, "Vibration response and wave propagation in periodic structures," Trans. Am. Soctety of Mech. Eng., J. Eng. Industry, vol. 93, ser. B, pp. 783-792, 1971 .

[245] J. C. Slater, "Interaction of waves in crystals" Rev. Mod. Phys., vol. $30, \mathrm{pp} .197-222,158$.

[246] C. Kittel, Introduction to Soltd State Physics. New York: J. Wiley and Sons, 1968.

[247] L. Esaki and L. L. Chang, "New transpor phenomenon in a semiconductor superlattice," Phys. Rev. Lett., vol. 33, pp. $495-498,1974$

[248] R. Tsu, L. L. Chang, G. Sai-Halasz, and L. Esaki, "Effects of quantum states on the photocurrent in a superlattice," Phys. Rev. Lett., vol. 34, pp. $1509-1512,1975$.

[249] J. P. Van der Ziel, R. Dingle, R. C. Miller, W. Wiegmann, and W. A. Nordland, "Laser oscillation from quantum states in very thin GaAs-AlGaAs multilayer structures," Appl. Phys. Lett., vol. 26 , pp. 463-465, 1975 .

[250] R. Dingle, A. C. Gossard, and W. Wiegmann, "Direct observa. tion of superiattice formation in a semiconductor heterostructure," Phys. Rev. Lett., vol. 34, pp. 1327-1330, 1975.

[251] K. Fischer and W. M. Meier, Fortschr, Mineral, vol. 42, p. 50, 1965.

[252] W. M. Meier, SCI Monograph on Molecular Sieves, vol. 10, 1968.

[253] E. H. Kerner, "Periodic impurities in a periodic lattice," Phys. Rev., vol. 95, pp. 687-689, 1954 .

[254] L. Dowrin, "Existence of energy gaps in the spectrum of a onedimensional atomic chain," Phys. Rev., vol. 138A, pp. 1121 1126,1965

[255] R. Landauer and L. C. Hellund, "Electronic structure of dis- 
ordered one-dimensional chains," J. Chem. Phys., vol. 22, pp. $1655-1665,1959$

[256] J. Hori, "Phase theory of disordered systems," Progr. Theoret. Phys. (Kyoto) Suppl. 36, pp. 3-54, 1966.

[257] N. F. Mott, "Electrons in disordered structures," Adv. Phys., vol. 16, pp. 49-144,1967.

[258] M. M. Pant and S. K. Joshi, "Green's function method for energy bands in disordered alloys: Application to alpha brass," Phys. Rev., vol. 186, pp. 675-682, 1969.

[259] H. I. Smith, "Fabrication techniques for surface acoustic wave and thin film optical devices," Proc. IEEE, vol. 62, pp. 1361 1387,1974 .

[260] D. Rudolph and G. Schmahl, "Verfahren sur herstellung von pontgenlinsen und beugungsgittern," Umschau Wiss. Tech., vol. 67, p. $225,1967$.

[261] S. E. Bernacki and H. I. Smith, "X-ray lithography applied to silicon device fabrication," Proc. of 6th Conf. on Elect. and Ion Beam Science and Technology, San Francisco, May, 1974.

[262] G. K. Wehner and G. S. Anderson, "The nature of physical sputtering," in Handbook of Thin Film Technology, L. I. Maissel and R. Glang, Eds. New York: McGraw-Hill, 1970.

[263] J. F. Gibbons, "Ion implantation in semiconductors, Part I Range distribution theory and experiments," Proc. IEEE, vol. 56, pp. 295-319, 1968 .

[264] - "Ion implantation in semiconductors, Part II: Damage production and annealing," Proc. IEEE, vol. 60, pp. 1062-1096, 1972.

[265] H. H. Schloessin, "Corrugation on the core boundary interfaces due to constitutional supercooling and effects on motion in 8 predominantiy stratified liquid core," Phys. Earth and Planet. Interiors, vol. 9, pp. 147-156, 1974.

[266] W. F. Utlaut and R. Cohen, "Modifying the ionosphere with intense radio waves," Science, vol. 174, pp. 245-254, 1971.

\section{Recent References and References Which Were Brought to the Attention of the Author by Reviewers.}

[267] Special Issue on the 1974 Semiconductor Laser Conference, IEEE J. Quantum Electron., vol. QE-11, 1975. Contains a number of papers on semiconductor DFB lasers.

[268] K. Asakawa et al., "Multimode Bragg diffractions in an ionexchanged optical waveguide with a sputter-etched periodic surface corrugation," IEEE J. Quantum Electron., vol. QE-11, pp. 304-306, 1975 .

[269] G. B. Inglis and F. Williams, "Electronic states of semiconductors with graded periodic inhomogeneities," Phys. Rev. B., vol. 11, pp. 2200-2204, 1975.

[270] K. P. Das, "Wave propagation in a plasma in the presence of a spatially uniform external periodic magnetic field," $J$. Flasma
Phys, vol. 13, pp. 327-334, 1975.

[271] V. Twersky, "Multiple scattering of waves by the doubly periodic planar array of obstacles," J. Math. Phys., vol. 16, pp. 633-643, 1975 .

[272] H. K. Parhi and A. K. Das, "Note on the effect of couple-stress on the waves emanating from periodic sources (Seismic Waves)," Gerlands Beitr. Geophys., vol. 83, pp. 481-484, 1974.

[273] R. M. Christensen, "Wave propagation in elastic media with a periodic array of discrete inclusions," $J$. Acoust. Soc. Amer, vol, 55, pp. 700-707, 1974 .

[274] G. N. Balanis, "Waves in a periodic composite," Trans ASME ser. E, vol. 40 , pp. 815-817, 1973.

[275] J. A. E. Calatroni and M. Garavaglia, "New analysis of the theory of rowland ghosts," Appl. Opt., vol. 12, pp. 2298$2301,1973$.

[276] A. C. Baynham et al., "Wave propagation in multilayered drifted solid-state plasmas," IEEE Trans. Microwave Theory Tech. vol. MTT-21, pp. 111-113, 1973 .

[277] U. R. Kristiansen and F, J. Fahy, "Scattering of acoustic waves by an N-layer periodic grating," $J$, Sound Vibr., vol. 24, pp. 315-332, 1972 .

[278] T. Tamir and H. L. Bertoni, "Lateral displacement of optical beams at multilayered and periodic structures," J. Opt. Soc. $A m_{\text {. }}$ vol. 61, pp. 1397-1413,1971.

[279] G. S. Hobson, "A demonstration of wave-propagation in periodic lattices," Int. J. Elec. Eng. Educ., vol. 9, pp. 90-95, 1971.

[280] M. Cornacchia, "Wave propagation and transient beam loading alternating periodic structures," Nucl. Instrum. Meth., vol. 94, pp. 109-123, 1971.

[281] V. P. Ivanov, "An inverse diffraction problem in periodic and doubly periodic lattices," $Z$ h. Vychtst. Mat. Fiz,, vol. 11, pp. $266-271,1971$ (in Russian)

[282] K. P. Das, "Effect of spatially uniform external periodic mag. netic field on wave propagation through a hot electron plasma," J. Plasma Phys, (GB), vol. 5, pp. 151-159, 1971.

[283] R. C. Preston, "asymmetry in the diffraction pattern of a grating due to periodic errors," Opt. Acta (GB), vol. 17, pp. 857867,1970 .

[284] A. C. Scott, "Tunnel diode arrays for information processing and storage," Trans. IEEE Systems, Man, Cybern., vol. SMC-1, p. 267,1971 .

[285] L. S. Hoel, W. H. Keller, J. E. Nordman, and A. C. Scott, "Niobium superconductive tunnel diode integrated circuit arrays," Solid-State Electron., vol. 15, pp. 1167-1173, 1972.

[286] J.P. Montgomery, "Scattering by an infinite periodic array of thin conductors on a dielectric sheet," IEEE Trans. Antennas Propagat., vol. AP-23, pp. 70-75, 1975.

[287] R. S. Chu and T. Tamir, "Bragg diffraction of Gaussian beams by periodically modulated media," J. Opt. Soc. Amer., vol. 66, pp. 220-226, 1976. 